\author{
Universidade de São Paulo \\ Instituto de Física
}

\title{
Estudo da Estrutura dos Novos Mésons
}

\author{
Raphael Moreira de Albuquerque
}

Dissertação de mestrado apresentada ao Instituto de Física da USP para a obtenção do título de Mestre em Ciências

Orientadora: Prof ${ }^{a}$. Dr ${ }^{a}$. Marina Nielsen

Banca Examinadora:

Profa. Dra. Marina Nielsen (USP)

Prof. Dr. Marcelo Chiapparini (UERJ)

Prof. Dr. Tobias Frederico (ITA) 


\section{FICHA CATALOGRÁFICA}

Preparada pelo Serviço de Biblioteca e Informação do Instituto de Física da Universidade de São Paulo

Albuquerque, Raphael Moreira de

Estudo da estrutura dos novos mésons. São Paulo, 2009.

Dissertação (Mestrado) - Universidade de São Paulo.

Instituto de Física - Departamento de Física Experimental

Orientador: Profa. Dra. Marina Nielsen

Área de Concentração: Física de Partículas

Unitermos: 1. Cromodinâmica quântica; 2. Física de partículas; 3. Física nuclear; 4. Física experimental;

5. Detecção de partículas. 
"Não fiques em terreno plano.

Não subas muito alto.

O mais belo olhar sobre o mundo

Está a meia altura."

Friedrich Nietzsche 



\section{Agradecimentos}

Aos meus queridos pais, Hilda e Ademir, por sempre estarem ao meu lado para me apoiar.

À minha amada Natalia. Seu amor e sua amizade, tornaram a minha vida mais completa.

À minha família, a base de tudo.

Ao brilhante grupo GRHAFITE: Arthur, Celso Barros, Celso Lima, Daniela, David, Diogo, Érike, Fabiana, Gabriel, Marina, Navarra, Olga, Patrícia, Paulo, Ricardo, Robilotta, Sérgio e Simone.

À Marina Nielsen pela sua orientação enérgica, objetiva, empolgante e sobretudo descontraída.

Ao Ricardo por me ajudar a desbravar o mundo das Regras de Soma. Seus conselhos foram essenciais para o desenvolvimento deste trabalho.

À minha querida irmã Dany, pelo carinho e pelo amor que mal pude aproveitar. Muitas saudades. Tchau, beijos... inté.

Ao apoio financeiro do Conselho Nacional de Desenvolvimento Científico e Tecnológico (CNPq). 



\section{Resumo}

Nós utilizamos as Regras de Soma da QCD para estudar a natureza dos mésons observados recentemente $Y(4260), Y(4360)$ e $Y(4660)$, e que são candidatos a estados exóticos de tetraquark $(c \bar{c} q \bar{q})$ ou $(c \bar{c} s \bar{s})$ com números quânticos $J^{P C}=1^{--}$. Nós trabalhamos com o termo dominante em $\alpha_{s}$, com as contribuições dos condensados de dimensão mais alta e com termos lineares da massa do quark estranho $m_{s}$. Nós obtivemos uma massa $m_{Y}=(4.65 \pm 0.11) \mathrm{GeV}$ para o estado $(c \bar{c} s \bar{s})$ que é compatível com o valor experimental do $Y(4660)$, enquanto que para o estado $(c \bar{c} q \bar{q})$ nós obtivemos uma massa $m_{Y}=(4.49 \pm 0.11) \mathrm{GeV}$, que também é consistente com o valor experimental do $Y(4360)$. Com a estrutura de tetraquark não foi possível explicar o $Y(4260)$.

Nós também consideramos os estados moleculares $D_{s 0} \bar{D}_{s}^{*}$ e $D_{0} \bar{D}^{*}$. Para a molécula $D_{s 0} \bar{D}_{s}^{*}$ nós obtivemos uma massa $m_{D_{s 0} \bar{D}_{s}^{*}}=(4.42 \pm 0.10) \mathrm{GeV}$ que é consistente, considerando a incerteza, com a massa do méson $Y(4360)$ e para a molécula $D_{0} \bar{D}^{*}$ obtivemos $m_{D_{0} \bar{D}^{*}}=(4.27 \pm 0.10) \mathrm{GeV}$ em excelente acordo com a massa do méson $Y(4260)$. 



\begin{abstract}
We use QCD sum rules to test the nature of the recently observed mesons $Y(4260), Y(4360)$ and $Y(4660)$, assumed to be exotic four-quark $(c \bar{c} q \bar{q})$ or $(c \bar{c} s \bar{s})$ states with $J^{P C}=1^{--}$. We work at leading order in $\alpha_{s}$, consider the contributions of higher dimension condensates and keep terms which are linear in the strange quark mass $m_{s}$. We find for the $(c \bar{c} s \bar{s})$ state a mass $m_{Y}=(4.65 \pm 0.10) \mathrm{GeV}$ which is compatible with the experimental candidate $Y(4660)$, while for the $(c \bar{c} q \bar{q})$ state we find a mass $m_{Y}=(4.49 \pm 0.11) \mathrm{GeV}$, which is still consistent with the mass of the experimental candidate $Y(4360)$. With the tetraquark structure we are working we can not explain the $Y(4260)$ as a tetraquark state.

We also consider molecular $D_{s 0} \bar{D}_{s}^{*}$ and $D_{0} \bar{D}^{*}$ states. For the $D_{s 0} \bar{D}_{s}^{*}$ molecular state we get $m_{D_{s 0} \bar{D}_{s}^{*}}=(4.42 \pm 0.10) \mathrm{GeV}$ which is consistent, considering the errors, with the mass of the meson $Y(4360)$ and for the $D_{0} \bar{D}^{*}$ molecular state we get $m_{D_{0} \bar{D}^{*}}=(4.27 \pm 0.10) \mathrm{GeV}$ in excelent agreement with the mass of the meson $Y(4260)$.
\end{abstract}





\section{Sumário}

1 Introdução 1

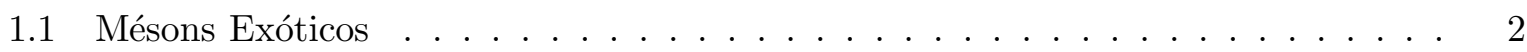

2 Regras de Soma da QCD (QCDSR) $\quad 7$

2.1 Função de Correlação . . . . . . . . . . . . . . . . . . . . . . 7

2.2 Lado da QCD . . . . . . . . . . . . . . . . . . . . . . . . . 9 9

2.2.1 Contribuições Perturbativas . . . . . . . . . . . . . . . . . 11

2.2.2 Contribuições não-Perturbativas para os Quarks Leves . . . . . . . . . . . 12

2.2.3 Contribuições não-Perturbativas para os Quarks Pesados . . . . . . . . . . 15

2.2 .4 Contribuições não-Fatorizáveis . . . . . . . . . . . . . . . . . 15

2.2.5 Resumo das Contribuições da OPE . . . . . . . . . . . . . . . . . . 17

2.3 Lado Fenomenológico . . . . . . . . . . . . . . . . . . . . . . . . . . . . 18

2.4 Relação de Dispersão . . . . . . . . . . . . . . . . . . . . . . . . . . . . . . 19

2.5 Transformada de Borel . . . . . . . . . . . . . . . . . . . . . . . . . . 21

2.5.1 Janela de Borel . . . . . . . . . . . . . . . . . . . . . . . . . . . 22

2.6 Princípio da Dualidade Quark-Hádron . . . . . . . . . . . . . . . . . 23

3 Família de Mésons Y em QCDSR $\quad 25$

3.1 Os Estados de Tetraquarks . . . . . . . . . . . . . . . . . 25

3.1.1 Transformações de Paridade e Conjugação de Carga . . . . . . . . . . . . 26

3.1 .2 Lado Fenomenológico . . . . . . . . . . . . . . . . . . . . 27

3.1 .3 Lado da QCD . . . . . . . . . . . . . . . . . . . . . 27

3.1.4 Dualidade Quark-Hadron . . . . . . . . . . . . . . . . . 34

3.1.5 Resultados Numéricos . . . . . . . . . . . . . . . . . . . . . 35

3.2 O Estado Molecular . . . . . . . . . . . . . . . . . . . . . . . . 39

3.2.1 Tranformações de Paridade e Conjugação de Carga . . . . . . . . . . . . . 39

3.2 .2 Lado Fenomenológico . . . . . . . . . . . . . . . . . . . . . . . . . 40

3.2 .3 Lado da QCD . . . . . . . . . . . . . . . . . . . . . . . . . . 41

3.2.4 Dualidade Quark-Hadron . . . . . . . . . . . . . . . . . . 44

3.2 .5 Resultados Numéricos . . . . . . . . . . . . . . . . . . . 45

$\begin{array}{lll}4 \text { Conclusões } & 49\end{array}$ 
A Propagadores Utilizados nas QCDSR $\quad \mathbf{5 1}$

A.1 Propagadores dos Quarks Leves . . . . . . . . . . . . . . . . . . 52

A.2 Propagadores dos Quarks Pesados . . . . . . . . . . . . . . . 53

B Fórmulas Úteis para as QCDSR

B.1 Transformadas de Fourier . . . . . . . . . . . . . . . . . . . 55

B.2 Álgebra das Matrizes de Gell-Mann . . . . . . . . . . . . . . . . . . 55

B.3 Álgebra das Matrizes de Dirac . . . . . . . . . . . . . . . . . . 55

B.4 Cálculo dos Traços . . . . . . . . . . . . . . . . . . . 56

B.5 Outras Relações Importantes . . . . . . . . . . . . . . . . . . . . . . . 57

B.6 Resolução das Integrais . . . . . . . . . . . . . . . . . . . . . . 58

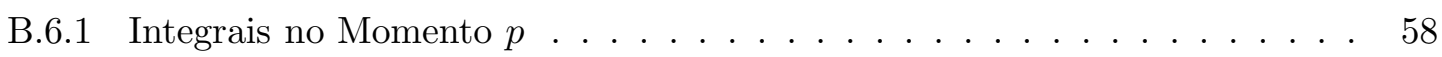

B.6.2 Integrais na Coordenada $x \ldots \ldots \ldots \ldots \ldots \ldots$

B.6.3 Integral $I_{n} \ldots \ldots \ldots \ldots \ldots \ldots \ldots \ldots$

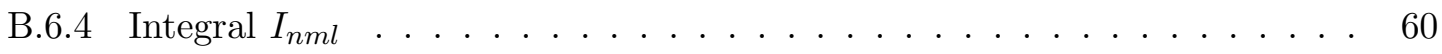

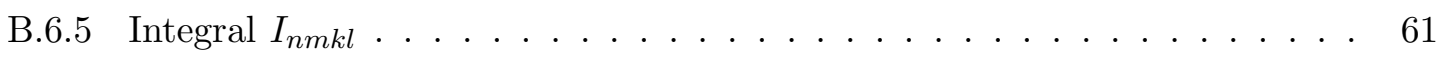

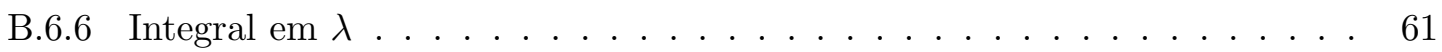

$\begin{array}{ll}\text { C Cálculo dos Diagramas de Tetraquarks } & 63\end{array}$

C.1 Contribuição Perturbativa . . . . . . . . . . . . . . . . . . 63

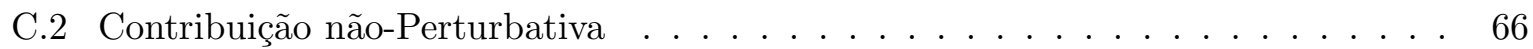

C.3 Contribuição não-Fatorizável . . . . . . . . . . . . . . . . . 68

$\begin{array}{ll}\text { D Cálculo dos Diagramas Moleculares } & \mathbf{7 1}\end{array}$

D.1 Contribuição Perturbativa . . . . . . . . . . . . . . . . . . . . . . 71

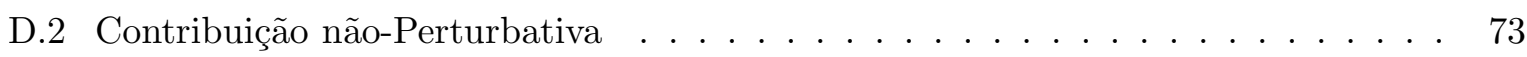

D.3 Contribuição não-Fatorizável . . . . . . . . . . . . . . . . . . . 75 


\section{Lista de Figuras}

1.1 Espectros de massa invariante: $J / \psi \pi^{+} \pi^{-}$e $\psi(2 S) \pi^{+} \pi^{-}$em uma produção ISR [10, 32]. 4

1.2 Distribuição da massa invariante do dipíon nos decaimentos: $Y(4260) \rightarrow J / \psi \pi^{+} \pi^{-}$ (esquerda), $Y(4360) \rightarrow \psi^{\prime} \pi^{+} \pi^{-}$(centro) e $Y(4660) \rightarrow \psi^{\prime} \pi^{+} \pi^{-}$(direita) [32]. . . . . . 4

2.1 Polarização do vácuo da QED. . . . . . . . . . . . . . . . . . . . . 8

2.2 Alguns diagramas de Feynman da QCD. . . . . . . . . . . . . . . . . . . . . . . 9

2.3 Contibuições não-perturbativas da QCD. . . . . . . . . . . . . . . . . . . . . 9

$2.4 \mathrm{O}$ contorno escolhido no plano complexo da variável $z=q^{2}$. O ponto aberto corresponde ao estado fundamental, para $q^{2}<0$. E as posições dos estados ressonantes, para $q^{2}>0$,

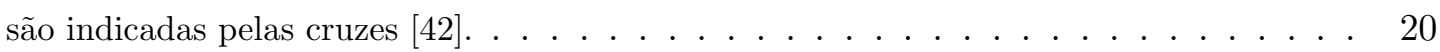

3.1 Relação Pólo x Contínuo, para $\sqrt{s_{0}}=5.1 \mathrm{GeV}$. . . . . . . . . . . . . . . . . 35

3.2 A convergência da OPE utilizando $\sqrt{s_{0}}=5.1 \mathrm{GeV}$. . . . . . . . . . . . . . . . . 36

3.3 A massa do tetraquark com estranheza em função do parâmetro $M^{2}$, para diferentes valores de $\sqrt{s_{0}}$. As setas delimitam os limites da janela de Borel das QCDSR. O limite inferior é dado pela condição de convergência da OPE e o limite superior é dado pela dominância da contribuição do pólo. . . . . . . . . . . . . . . . . . . . . 36

3.4 Relação Pólo x Contínuo, para $\sqrt{s_{0}}=4.9 \mathrm{GeV}$. . . . . . . . . . . . . . . . . . . 37

3.5 A convergência da OPE utilizando $\sqrt{s_{0}}=4.9 \mathrm{GeV}$. . . . . . . . . . . . . . . . . . . 38

3.6 A massa do tetraquark com quark leve em função do parâmetro $M^{2}$, para diferentes valores de $\sqrt{s_{0}}$. As setas delimitam a janela de Borel das QCDSR. . . . . . . . . . . 39

3.7 Relação Pólo x Contínuo, para $\sqrt{s_{0}}=4.9 \mathrm{GeV}$. . . . . . . . . . . . . . . . . . . . 45

3.8 A convergência da OPE utilizando $\sqrt{s_{0}}=4.9 \mathrm{GeV}$. . . . . . . . . . . . . . . . 46

3.9 A massa da Molécula $D_{s 0} \bar{D}_{s}^{*}$ em função do parâmetro $M^{2}$, para diferentes valores de $\sqrt{s_{0}}$. As setas delimitam a janela de Borel das QCDSR. . . . . . . . . . . . . . . . 46

3.10 Relação Pólo x Contínuo, para $\sqrt{s_{0}}=4.7 \mathrm{GeV}$. . . . . . . . . . . . . . . . . 47

3.11 A convergência da OPE utilizando $\sqrt{s_{0}}=4.7 \mathrm{GeV}$. . . . . . . . . . . . . . . 47

3.12 A massa da Molécula $D_{0} \bar{D}^{*}$ em função do parâmetro $M^{2}$, para diferentes valores de $\sqrt{s_{0}}$. As setas delimitam a janela de Borel das QCDSR. . . . . . . . . . . . . . . . . 48 



\section{Capítulo 1}

\section{Introdução}

As interações fortes, responsáveis por formarem os hádrons (mésons e bárions) a partir de estados ligados de quarks, são descritas por uma teoria de campo não-abeliana chamada Cromodinâmica Quântica (QCD, do inglês Quantum ChromoDynamics) [1]. Na QCD, as únicas partículas a sentirem os efeitos da interação forte são aquelas que possuem a chamada carga de cor. Com esta carga, as partículas tornam-se "coloridas" e interagem via troca de glúons. Note a analogia que há com a Eletrodinâmica Quântica (QED, do inglês Quantum ElectroDynamics), onde as partículas carregadas eletromagneticamente interagem por meio da troca de fótons [1]. No entanto, a QCD apresenta algumas propriedades fundamentais que a torna uma teoria muito mais complexa do que a QED. Destas propriedades podemos citar as mais importantes: a liberdade assintótica e o confinamento.

O princípio da liberdade assintótica nas interações fortes é caracterizada pelo comportamento da constante de acoplamento da QCD, $g_{s}$, em altas energias. Neste caso, a QCD se torna uma teoria fracamente acoplada $\left(g_{s} \longrightarrow 0\right)$ e pode ser avaliada através de métodos perturbativos que descrevem, com uma boa aproximação, os processos de espalhamento que envolvam as interações fortes. Entretanto, no limite de baixas energias, verificamos que a QCD se torna uma teoria fortemente acoplada $\left(g_{s}>>1\right)$ e seu regime de aplicabilidade é dominado por efeitos altamente não-perturbativos. Com isto, há grandes dificuldades em se determinar completamente o espectro hadrônico partindo-se da QCD. Espera-se que com o avanço computacional, os cálculos numéricos da QCD na Rede [2] possam confirmar os valores de observáveis tais como massas e constantes de decaimentos. Porém, como o progresso da QCD na Rede é feita de forma bastante gradual, ainda será necessário muito tempo para termos uma confirmação da validade de aplicabilidade da QCD.

Vários modelos, que são construídos incorporando as propriedades fundamentais da QCD, têm se mostrado muito úteis e confiáveis na descrição do espectro e das propriedades dos hádrons. Um deles, inspirado na proposta feita, independentemente, pelos físicos Murray Gell-Mann [3] e George Zweig [4], que é amplamente utilizado para classificar os hádrons e calcular suas massas e constantes de decaimentos [5], é o Modelo dos Quarks Constituintes (CQM, do inglês Constituent Quark Model). De acordo com o CQM, os mésons são compostos de um par quark-antiquark $(q \bar{q})$, enquanto que os bárions são compostos por um tripleto de quarks (qqq). Para serem observáveis físicos no CQM, as partículas devem ser estados singletos de cor (carga de cor nula). 
Desta forma, mésons e bárions são formados por combinações de quarks que neutralizam a carga de cor. Note que, os quarks são partículas fundamentais carregadas com cor e, desta forma não podem ser observados em estados livres. Este é o princípio do confinamento, que garante que os quarks ficam confinados no interior dos hádrons.

Vale ressaltar que, além das estruturas convencionais $q \bar{q}$ e $q q q$ do CQM, a QCD permite a existência das mais variadas estruturas internas para os hádrons como: glueballs ( $g g g)$ [6], estados híbridos $(q \bar{q} g)[7]$, moléculas hadrônicas $\left(D_{0}-\bar{D}^{*}\right)[8]$, estados de multiquarks $(q q \bar{q} \bar{q})$ [9], etc.

De fato, embora o quadro atual da Física de Partículas revele que a grande maioria dos hádrons observados sejam muito bem descritos pelo CQM, recentes descobertas sobre o espectro do charmonium (méson $c \bar{c}$ ), realizadas pelas colaborações BaBar [10] e Belle [11], têm indicado a possível existência de mésons com uma subestrutura muito mais complexa do que $q \bar{q}$. Como não se sabe se esses estados realmente existem convencionou-se chamá-los de mésons exóticos.

\subsection{Mésons Exóticos}

As recentes descobertas extraordinárias sobre a espectroscopia do charmonium foram obtidas pelas chamadas B-Factories: a PEPII no Centro do Acelerador Linear de Stanford (SLAC) [10], nos Estados Unidos, e a KEKB na Organização de Pesquisas em Aceleradores de Altas Energias (KEK) [11], no Japão. Originalmente, as $B$-Factories foram construídas para investigar a violação da simetria CP nos decaimentos dos mésons $B$. Essas $B$-Factories são "colliders" de elétrons e pósitrons, trabalhando numa energia de centro de massa em torno de $10.58 \mathrm{GeV}$. A aniquilação de elétrons e pósitrons nessas energias produzem um número muito grande de mésons $B$ e $\bar{B}$. Os pares $B \bar{B}$ produzidos são medidos pelas colaborações Belle (no KEKB) e BaBar (no PEPII). Um bônus inesperado dos experimentos das $B$-Factories tem sido a observação de novas ressonâncias, cujas massas não estão de acordo com as previsões dos modelos de quarks constituintes.

Tudo começou em 2003, quando a colaboração BaBar anunciou a descoberta do estado estreito, $D_{s J}^{+}(2317)$, decaindo em $D_{s}^{+} \pi^{0}$ [12]. Este processo logo foi confirmado pela colaboração CLEO da Universidade de Cornell que, em seguida, anunciou a descoberta de um outro estado estreito, $D_{s J}^{+}(2460)$, decaindo em $D_{s}^{*+} \pi^{0}$ [13]. Esses estados foram interpretados como os estados $D_{s}^{*}$ de spin, paridade e conjugação de carga $0^{++}$e $1^{++}$, respectivamente. Entretanto, estes novos estados são bem mais leves e estreitos do que a previsão do CQM. Devido a isso, a colaboração BaBar sugeriu que o estado $D_{s J}^{+}(2317)$ poderia ser um estado de tetraquark $q \bar{q} c \bar{s}$.

Seguindo estas descobertas, a colaboração Belle, anunciou sua descoberta do méson $X(3872)$, observado no decaimento:

$$
B^{+} \longrightarrow X(3872) K^{+} \longrightarrow J / \psi \pi^{+} \pi^{-} K^{+}
$$

O méson $X(3872)$ foi rapidamente confirmado pelas colaborações CDF II [14] e D0 [15] que o observaram em colisões à altas energias entre próton-antipróton, no Fermilab Tevatron. Sua produção também foi confirmada, nos decaimentos dos mésons B, pela colaboração BaBar [12]. Atualmente, o valor da sua massa é dada por $(3871.4 \pm 0.6) \mathrm{MeV}$, e sua largura total é menor 
do que $2.3 \mathrm{MeV}[16]$.

Ambas as colaborações, BaBar [17] e Belle [18], observaram o canal de decaimento $X(3872) \longrightarrow$ $\gamma J / \psi$, o que indica que o méson $X(3872)$ tenha conjugação de carga dada por $C=+$, em oposição a conjugação de carga dos candidatos convencionais do charmonium. Em [18] também foi relatada a observação do decaimento $X(3872) \longrightarrow J / \psi \pi^{+} \pi^{-} \pi^{0}$, com uma taxa comparável com a do modo $X(3872) \longrightarrow J / \psi \pi^{+} \pi^{-}$, que estabelece uma forte violação do isospin. Finalmente, uma análise da distribuição angular sugere os números quânticos $J^{P C}=1^{++}[19]$.

De acordo com CQM, o único estado do espectro do charmonium com uma massa em torno de $3900 \mathrm{MeV}$ e com números quânticos $J^{P C}=1^{++}$, corresponde ao $\chi_{1}^{\prime}(3925)$ [20]. Portanto, com esta diferença entre as massas $(\sim 50 \mathrm{MeV})$, o $X(3872)$ não pode ser identificado como um estado convencional do charmonium. Muitas propostas foram feitas acerca da estrutura do $X(3872)$ : tetraquark [9, 21, 22], cusp [23], híbrido [24], glueball [25] ou um estado molecular $D \bar{D}^{*}[26,27]$.

Em 2005, os experimentos que utilizavam processos com radiação do estado inicial (ISR, do inglês Initial State Radiation) realizados pela Colaboração BaBar, indicaram um pico de ressonância em torno de $4300 \mathrm{MeV}$, produzido na reação [28]:

$$
e^{+} e^{-} \longrightarrow \gamma_{I S R} Y(4260) \longrightarrow \gamma_{I S R} J / \psi \pi^{+} \pi^{-}
$$

A largura medida foi de aproximadamente $90 \mathrm{MeV}$.

Nesse tipo de processo ISR, o estado inicial, $e^{+}$ou $e^{-}$, irradia um raio- $\gamma$ muito energético, e o par $e^{+} e^{-}$subsequentemente se aniquila, numa energia reduzida. Quando a energia do centro de massa do fóton irradiado $\left(\gamma_{I S R}\right)$ está entre 4000 e $5000 \mathrm{MeV}$, a aniquilação $e^{+} e^{-}$ocorre numa energia que corresponde à região de massas dos estados do charmonium. Assim, esse processo ISR pode produzir estados do charmonium com os números quânticos: $J^{P C}=1^{--}$. Como o $Y(4260)$ é produzido via processos ISR, trata-se de um méson vetorial. O méson $Y(4260)$ foi confirmado também pela colaboração CLEO [29].

Nesta região de massa $(4000-4500 \mathrm{MeV})$, os estados $c \bar{c}$ convencionais do charmonium são bem estabelecidos: $\psi(4040)$ : estado $3^{3} S_{1}, \psi(4160)$ : estado $2^{3} D_{1}$ e $\psi(4415)$ : estado $4^{3} S_{1}$, cujas massas estão em excelente acordo com os modelos de potencial [20]. Esta observação levou alguns autores a considerar o $Y(4260)$ como um bom candidato a um estado de tetraquarks [30].

Recentemente, a colaboração Belle não só confirmou a existência do estado $Y(4260)$ [31], como também, anunciou a descoberta de mais dois outros mésons vetoriais [11], o $Y(4360)$ e o $Y$ (4660), cujos picos de ressonância estão em torno de $4360 \mathrm{MeV}$ e $4660 \mathrm{MeV}$, respectivamente. Ambos possuem os números quânticos $J^{P C}=1^{--}$e são produzidos na seguinte reação:

$$
e^{+} e^{-} \longrightarrow \gamma_{I S R} \psi^{\prime} \pi^{+} \pi^{-}
$$

As larguras do $Y(4360)$ e do $Y(4660)$ foram medidas e seus valores aproximados são, respectivamente, $74 \mathrm{MeV}$ e $48 \mathrm{MeV}$ [11]. Com estes valores para as massas e larguras, verificou-se que tambem os estados, $Y(4360)$ e $Y(4660)$, não são consistentes com estados convencionais $1^{--}$ do charmonium na região de massa $4000-5000 \mathrm{MeV}$. Os espectros de massa obtidos pelas colaborações BaBar e Belle são reproduzidos na figura (1.1). 

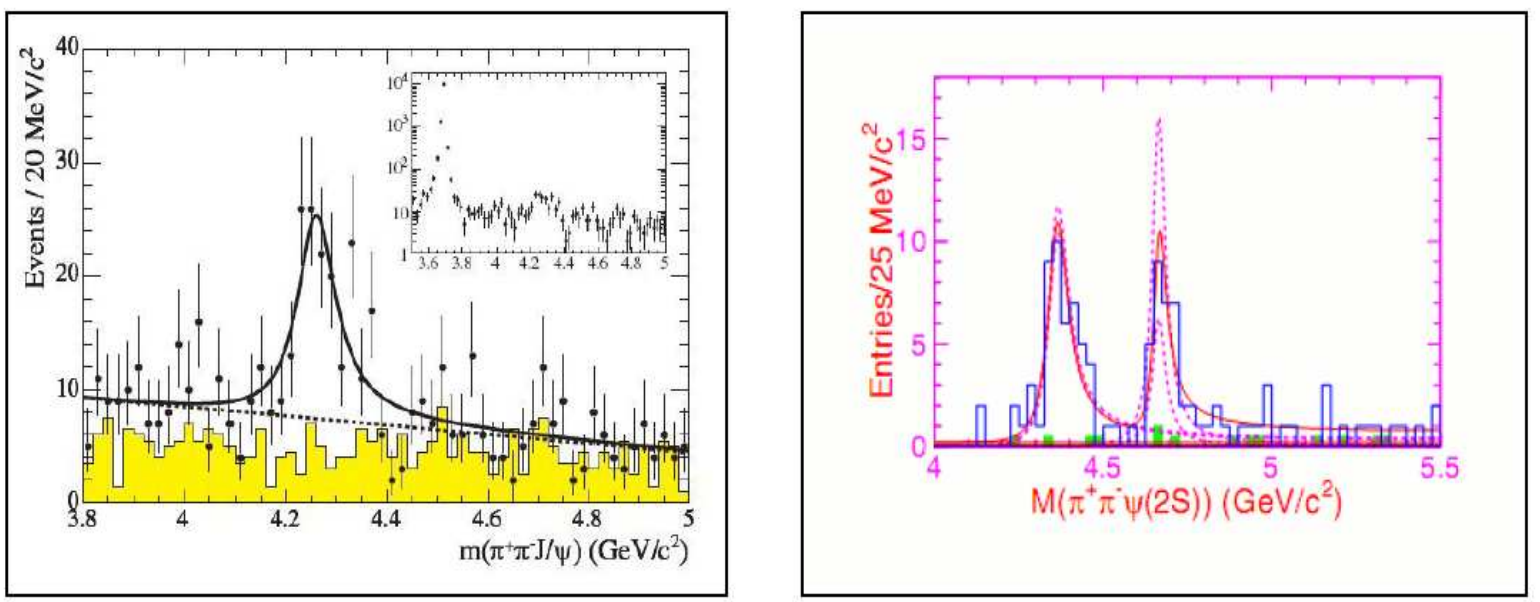

Figura 1.1: Espectros de massa invariante: $J / \psi \pi^{+} \pi^{-}$e $\psi(2 S) \pi^{+} \pi^{-}$em uma produção ISR [10,32].

Uma informação importante para entendermos a estrutura destes estados é se o par de píons, nos seus decaimentos, são originários de um estado ressonante intermediário. Do espectro de massa invariante do dipíon, mostrado na ref.[32] e aqui reproduzido na figura (1.2), verifica-se que apenas o $Y(4660)$ tem um estado intermediário bem definido e consistente com $f_{0}(980)$ [32]. Devido a este fato e a proximidade da massa do sistema $\psi^{\prime}-f_{0}(980)$ com a massa do méson $Y(4660)$, na ref. [33], o $Y(4660)$ foi considerado como um estado ligado $f_{0}(980) \psi^{\prime}$. Há também outras interpretações para o $Y(4660)$ como a que o considera um estado bariônico [34] ou um estado canônico $5^{3} S_{1} c \bar{c}$ [35]. Para os outros dois mésons, $Y(4260)$ e $Y(4360)$, a figura (1.2) indica que eles são mais consistentes com o méson escalar $\sigma[36,32]$ do que com o $f_{0}(980)$. Há também a proposta de Maiani et al [30], em que o estado de tetraquark [csc̄s] corresponderia ao méson $Y(4260)$.
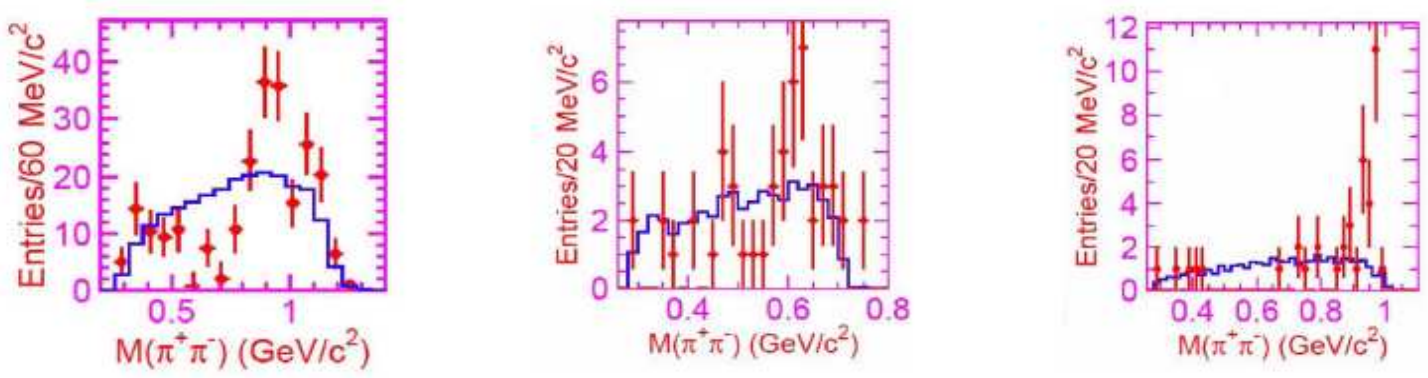

Figura 1.2: Distribuição da massa invariante do dipíon nos decaimentos: $Y(4260) \rightarrow J / \psi \pi^{+} \pi^{-}$(esquerda), $Y(4360) \rightarrow \psi^{\prime} \pi^{+} \pi^{-}$(centro) e $Y(4660) \rightarrow \psi^{\prime} \pi^{+} \pi^{-}$(direita) [32].

Nas seções seguintes pretendemos investigar a possível estrutura de quarks dos mésons vetoriais charmosos $Y(4260)$, $Y(4360)$ e $Y(4660)$ utilizando as Regras de Soma da QCD (QCDSR, 
do inglês QCD Sum Rules) [37]. As QCDSR já foram empregadas, por exemplo, no estudo das partículas $X(3872)$ [38] e $Z^{+}$(4430) [39], e os resultados obtidos estão em bom acordo com o valor experimental.

Usaremos as QCDSR para calcular as massas dos seguintes estados tetraquarks e moleculares:

- tetraquark com quarks leves: $[c q \bar{c} \bar{q}]$, onde $q=u, d$.

- tetraquark com estranheza: $[c s \bar{c} \bar{s}]$.

- molécula com quarks leves: $[c \bar{q}][q \bar{c}]$.

- molécula com estranheza: $[c \bar{s}][s \bar{c}]$.

Comparando os resultados obtidos com os valores experimentais das massas dos mésons $Y(4260)$, $Y(4360)$ e $Y(4660)$, tentaremos relacioná-los com a correspondente estrutura de tetraquark ou molécula. 



\section{Capítulo 2}

\section{Regras de Soma da QCD (QCDSR)}

O método das QCDSR, originalmente proposto por Shifman, Vainshtein e Zakharov [37], é baseado na comparação entre duas descrições das funções de correlação hadrônicas: no lado teórico das regras de soma a função de correlação é avaliada usando a expansão dos operadores de Wilson (OPE) em termos dos campos de quarks e glúons; já no lado fenomenológico, a representação espectral da função de correlação é parametrizada em termos de propriedades hadrônicas. Os valores dos observáveis hadrônicos são obtidos através da comparação entre estas duas descrições que, segundo o princípio da dualidade, são equivalentes .

Os efeitos não perturbativos, devido ao vácuo físico da QCD, são introduzidos através dos condensados de quarks e glúons. A introdução destes efeitos e o uso da Transformada de Borel é o que irá permitir estender o domínio padrão da QCD perturbativa para valores de $Q^{2}$ da ordem da massa do núcleon.

A precisão deste método é limitada por aproximações da função de correlação nos dois lados (QCD e Fenomenológico). Assim, as Regras de Soma devem ser estudadas caso a caso para determinar seus limites de validade e margens de erro.

As seções seguintes mostrarão com mais detalhes as características das Regras de Soma mais relevantes para este trabalho, identificando os passos necessários para a sua aplicação na determinação das massas dos hádrons.

\subsection{Função de Correlação}

A função de correlação desempenha um papel fundamental nas QCDSR. A partir dela, é que conseguimos extrair os parâmetros do estado fundamental do hádron a ser estudado, como por exemplo, a sua massa. Ao estudarmos um hádron vetorial, utilizamos a função de correlação de dois pontos definida por:

$$
\Pi_{\mu \nu}(q)=i \int d^{4} x e^{i q \cdot x}\left\langle 0\left|T\left[j_{\mu}(x) j_{\nu}^{\dagger}(0)\right]\right| 0\right\rangle
$$

onde $j_{\mu}(x)$ é a corrente hadrônica que deve reproduzir os mesmos números quânticos do hádron em questão e $q$ é o seu quadrimomento total com $q^{2}<0$.

Ao lidarmos com correntes hadrônicas conservadas, podemos escrever (2.1) com a seguinte 
estrutura de Lorentz:

$$
\Pi_{\mu \nu}(q)=\left(q_{\mu} q_{\nu}-q^{2} g_{\mu \nu}\right) \Pi\left(q^{2}\right)
$$

onde $\Pi\left(q^{2}\right)$ é uma função analítica que contém todos os efeitos dinâmicos do hádron. Contraindo os índices de Lorentz, obtemos a relação entre a função de correlação e a função analítica $\Pi\left(q^{2}\right)$ :

$$
\Pi_{\mu}^{\mu}(q)=-3 q^{2} \Pi\left(q^{2}\right)
$$

Para nos familiarizar com este objeto, vamos considerar a QED. Neste caso, $j_{\mu}(x)$ é identificada com a corrente vetorial de Dirac: $\bar{\Psi}(x) \gamma_{\mu} \Psi(x)$ que se acopla com o campo eletromagnético $A^{\mu}(x)$.

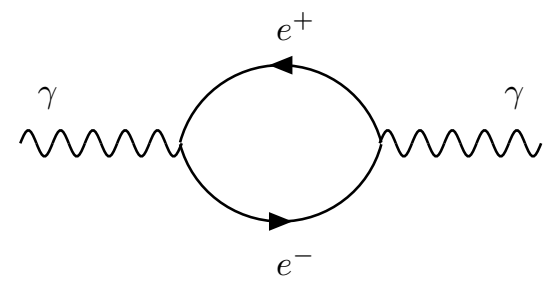

Figura 2.1: Polarização do vácuo da QED.

O diagrama de polarização do vácuo da QED [1] ilustra o processo calculado em (2.1). Uma corrente da forma $\bar{\Psi} \gamma_{\mu} \Psi$, mas criando e aniquilando quarks, deve ser conveniente para o estudo dos mésons, desde que o estado intermediário $\bar{q} q$ seja visto como acoplado com uma série completa de estados mesônicos. Assim, queremos criar um par quark-antiquark no ponto 0 do espaço-tempo, propagá-lo no vácuo da QCD e aniquilá-lo em $x$.

Nas QCDSR a descrição da função de correlação é feita de duas formas distintas. De um lado calcula-se a função de correlação utilizando a corrente $j_{\mu}(x)$ em termos dos campos de quarks e glúons (Lado da QCD). Por outro lado, o cálculo é feito utilizando-se parâmetros hadrônicos (Lado Fenomenológico) como massa e a constante de acoplamento à corrente $j_{\mu}(x)$. No Lado da QCD, a estrutura complexa do vácuo leva-nos a empregar a técnica da Expansão em Produto de Operadores, ou OPE de Wilson. Essa expansão, além de permitir o uso das técnicas da QCD perturbativa, leva naturalmente à parametrização do vácuo da QCD em termos dos chamados condensados do vácuo, além disso, é possível manipular (2.1) de forma a obter uma expressão dependente, explicitamente, dos propagadores dos quarks contidos em $j_{\mu}(x)$. No Lado Fenomenológico, quantificamos o conteúdo hadrônico de (2.1) inserindo um conjunto completo de estados hadrônicos intermediários acoplados à corrente $j_{\mu}(x)$.

É na corrente $j_{\mu}(x)$ que inserimos as características do hádron a ser estudado, tais como números quânticos, conteúdo de quarks, parâmetros fenomenológicos, etc. Logo, sua construção torna-se o primeiro passo para lidarmos analiticamente com a função de correlação (2.1).

Por fim, para extraírmos os valores dos observáveis físicos, utilizando as QCDSR, comparamos os dois lados da teoria. Esta comparação é feita usando o Princípio da Dualidade QuarkHádron, que postula a existência de um intervalo onde um hádron pode ser equivalentemente descrito - tanto no Lado da QCD como no Lado Fenomenológico. 


\section{$2.2 \quad$ Lado da QCD}

Ao considerarmos valores de $x$ suficientemente pequenos (regime perturbativo), a Eq. (2.1) pode ser calculada pela soma dos diagramas de Feynman contidas na QCD perturbativa.

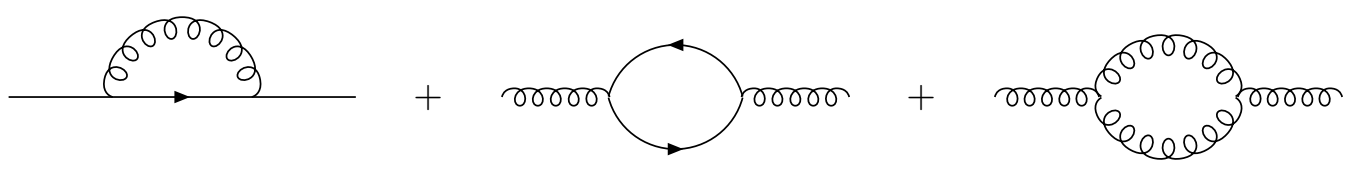

Figura 2.2: Alguns diagramas de Feynman da QCD.

A Fig.(2.2) ilustra as linhas cheia e ondulada que representam, respectivamente, os propagadores do quark e do glúon. Do princípio de incerteza de Heisenberg, pequenos valores de $x$ implicam que nós temos que considerar grandes momentos. Assim, se trabalharmos na região assintótica $\left(Q^{2} \rightarrow \infty\right)$ podemos usar expressões nuas para os propagadores de quarks e glúons. Entretanto, este limite assintótico não é numericamente razoável e temos que trabalhar com momentos da ordem de $Q^{2} \approx 1 \mathrm{GeV}^{2}$. Note que, essa ordem de momentos ainda é completamente razoável para o regime perturbativo:

$$
\frac{\alpha_{s}\left(1 \mathrm{GeV}^{2}\right)}{\pi} \approx 0.1-0.7
$$

Para sistemas cujo momento, $k$, é inferior ao ponto de renormalização, $\mu$, da QCD (onde $\mu \approx 0.5 \mathrm{GeV}$ ), estaremos cometendo erros grandes se usarmos os propagadores nus no cálculo dos diagramas de Feynman. Portanto, para $|k|>\mu$ nós podemos usar os propagadores nus, mas para $|k|<\mu$ nós não sabemos o que usar. Para tentar resolver este problema, podemos considerar que o momento que flui através de uma das linhas é grande se aproximarmos $|k|<\mu$ por $k=0$ na linha soft. Este procedimento é uma maneira de lidarmos com as contribuições de origem não-perturbativa, que graficamente podemos representá-las por:

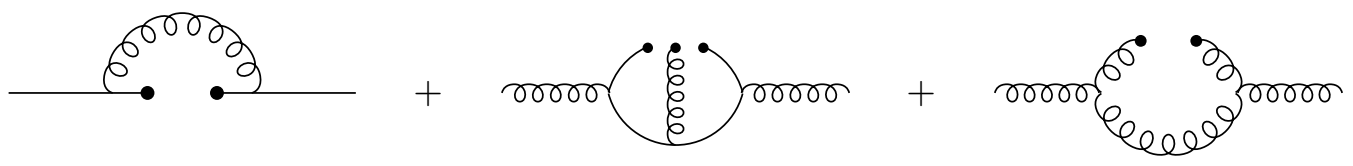

Figura 2.3: Contibuições não-perturbativas da QCD.

Dessa forma os condensados de quarks, glúons e quarks-glúons aparecem. Em geral, qualquer diagrama de Feynman pode ser preparado de forma a incorporar os condensados. Para este fim, é realizada a expansão da função de correlação em uma série de operadores locais, ou operadores de Wilson (OPE):

$$
\int d^{4} x e^{i q \cdot x}\left\langle 0\left|T\left[j_{\mu}(x) j^{\dagger \mu}(0)\right]\right| 0\right\rangle=\sum_{d} C_{d}\left(q^{2}\right) \hat{O}_{d}(0) .
$$

Os coeficientes $C_{d}$, chamados de coeficientes de Wilson, contêm os efeitos perturbativos da QCD 
à altas energias $(|k|>>\mu)$, enquanto que os operadores $\hat{O}_{d}$ são operadores locais compostos, construídos a partir de campos de quarks e glúons, e contêm os efeitos não-pertubativos da QCD à baixas energias $(|k|<\mu)$. O índice $d$ representa a dimensão do operador local.

Nas QCDSR, o operador de dimensão zero é dado por $\hat{O}_{0}=\hat{1}$ e, com isto, o seu coeficiente $C_{0}$ representa as contribuições de origem pertubativa. Os operadores locais de mais baixa ordem são dados por:

$$
\begin{aligned}
& \hat{O}_{3}=\bar{q} q \\
& \hat{O}_{4}=G_{\mu \nu}^{a} G_{a}^{\mu \nu} \\
& \hat{O}_{5}=\bar{q} \sigma_{\mu \nu} \frac{\lambda^{a}}{2} G_{a}^{\mu \nu} q \\
& \hat{O}_{6}=\left(\bar{q} \Gamma_{r} q\right)\left(\bar{q} \Gamma_{s} q\right)
\end{aligned}
$$

onde $q$ representa o campo dos quarks, $G_{\mu \nu}^{a}$ representa o campo dos glúons, $\lambda^{a}$ são as matrizes de Gell-Mann, $\sigma_{\mu \nu}=\frac{i}{2}\left[\gamma_{\mu}, \gamma_{\nu}\right]$ e $\Gamma_{r, s}$ é uma combinação de matrizes de Lorentz e de cor. Os valores esperados destes operadores locais, no vácuo da QCD, fornecem as expressões analíticas do condensado de quarks (2.5), do condensado de glúons (2.6), do condensado de quark-glúon (2.7) e do duplo condendado de quarks (2.8). Em geral, o uso dos operadores até dimensão 6 é suficiente para descrever a maioria dos processos físicos observáveis utilizando-se as QCDSR. A lista de operadores de dimensão mais altas $(d>6)$ e sua aplicabilidade pode ser encontrada em diversas referências [40, 42].

Os condensados introduzidos na OPE são parâmetros não-perturbativos, logo seus valores numéricos não podem ser calculados diretamente e têm que ser determinados por outros métodos. O valor numérico de $\langle\bar{q} q\rangle$ pode ser determinado, por exemplo, usando-se a hipótese de PCAC (Corrente Axial Parcialmente Conservada) [43]:

$$
\langle\bar{q} q\rangle=-\frac{m_{\pi}^{2} f_{\pi}^{2}}{2\left(m_{u}+m_{d}\right)}
$$

onde $m_{u}$ e $m_{d}$ são, respectivamente, as massas dos quarks $u$ e $d, m_{\pi}$ e $f_{\pi}$ são a massa e a constante de decaimento do píon. Utilizando os valores numéricos $m_{\pi}=138 \mathrm{MeV}, f_{\pi}=132 \mathrm{MeV}$ e tomando os valores usuais para as massas dos quarks, $m_{u}+m_{d} \cong 14 \mathrm{MeV}$, obtemos

$$
\langle\bar{q} q\rangle \cong-(0.228 \mathrm{GeV})^{3}
$$

O condensado de glúons $\left(\hat{O}_{4}\right)$ e o condensado de quarks-glúons $\left(\hat{O}_{5}\right)$ podem ser obtidos de forma análoga [41].

Neste trabalho os condensados são obtidos pela aplicação do teorema de Wick. Este procedimento expande um produto temporalmente ordenado de operadores de campo em função dos propagadores associados a estes operadores. Como exemplo, dado o produto ordenado no tempo de dois campos fermiônicos, $q_{i}(x)$ e $\bar{q}_{j}(0)$, temos que:

$$
\left\langle 0\left|T\left[q_{i}(x) \bar{q}_{j}(0)\right]\right| 0\right\rangle=\left\langle 0_{p}\left|T\left[q_{i}(x) \bar{q}_{j}(0)\right]\right| 0_{p}\right\rangle+\left\langle 0\left|: q_{i}(x) \bar{q}_{j}(0):\right| 0\right\rangle
$$


onde $\left|0_{p}\right\rangle$ representa o vácuo perturbativo, $|0\rangle$ o vácuo da $\mathrm{QCD}$ e $i, j=1,2,3$ são indíces de cor. O segundo termo representa a ordem normal dos operadores de campo de quarks. Note que, este termo seria zero no vácuo perturbativo, mas é justamente ele quem introduz contribuições não-perturbativas e não-fatorizáveis do vácuo da QCD no método das QCDSR.

Da definição $S_{a b}^{q}(x)=\left\langle 0\left|T\left[q_{a}(x) \bar{q}_{b}(0)\right]\right| 0\right\rangle$, logo a Eq. (2.11) pode ser reescrita como:

$$
S_{a b}^{q}(x)=S_{a b}^{0}(x)+\left\langle 0\left|: q_{a}(x) \bar{q}_{b}(0):\right| 0\right\rangle
$$

que é o propagador completo utilizado nas QCDSR.

\subsubsection{Contribuições Perturbativas}

O primeiro termo da Eq.(2.12) é o propagador perturbativo da QCD. Sua expressão - bem conhecida em Teoria Quântica de Campos (QFT) - é dada pela fórmula de Gell-Mann Low [1]:

$$
S_{a b}^{0}(x)=\left\langle 0_{p}\left|T\left[q_{a}(x) \bar{q}_{b}(0) e^{i \int d^{4} z \mathcal{L}_{i n t}(z)}\right]\right| 0_{p}\right\rangle
$$

onde a lagrangiana de interação entre quarks e glúons é dada por

$$
\mathcal{L}_{\text {int }}(z)=g_{s} \bar{q}^{a}(z) \gamma^{\mu} \tau_{a b}^{n} A_{\mu}^{n} q^{b}(z)
$$

e $g_{s}$ é a constante de acoplamento forte da QCD, $A_{\mu}^{n}$ representa o campo dos glúons, $\tau_{a b}^{n}$ são os geradores do grupo $S U(3)$ e $a=1,2, \ldots, 8$. Assim, expandindo a exponencial até a primeira ordem:

$$
S_{a b}^{0}(x)=\left\langle 0_{p}\left|T\left[q_{a}(x) \bar{q}_{b}(0)\right]\right| 0_{p}\right\rangle+i \int d^{4} z\left\langle 0_{p}\left|T\left[q_{a}(x) \bar{q}_{b}(0) \mathcal{L}_{\text {int }}(z)\right]\right| 0_{p}\right\rangle+\cdots
$$

que pode ser reescrita como

$$
S_{a b}^{0}(x) \equiv S_{a b}^{(0)}(x)+S_{a b}^{(1)}(x)+\cdots
$$

O primeiro termo desta equação é dado pelo propagador dos quarks livres:

$$
S_{a b}^{(0)}(x)=i \delta_{a b} \int \frac{d^{4} p}{(2 \pi)^{4}}\left(\frac{\not p+m_{q}}{p^{2}-m_{q}^{2}+i \epsilon}\right) e^{-i p \cdot x} .
$$

Já o segundo termo, representa o vértice de interação entre quarks e glúons e, de acordo com [44], é dado por:

$$
S_{a b}^{(1)}(x)=-\frac{i}{4} g_{s} \tau_{a b}^{n} G_{\mu \nu}^{n}(0) \int \frac{d^{4} p}{(2 \pi)^{4}}\left[\frac{\sigma^{\mu \nu} \not p+\not p \sigma^{\mu \nu}+2 m_{q} \sigma^{\mu \nu}}{\left(p^{2}-m_{q}^{2}+i \epsilon\right)^{2}}\right] e^{-i p \cdot x}
$$

onde $G_{\mu \nu}^{n}=\partial_{\mu} A_{\nu}^{n}-\partial_{\nu} A_{\mu}^{n}+g_{s} f_{j k}^{n} A_{\mu}^{j} A_{\nu}^{k}$ é o campo tensorial antissimétrico dos glúons.

Quando os propagadores contém apenas quarks leves $(u, d$ e $s)$, podemos fazer aproximações no limite em que $m_{q} \rightarrow 0$. Neste caso, é conveniente escrever as equações (2.17) e (2.18) no 
espaço das coordenadas. Utilizando as relações (B.2) e considerando termos em $1 \underline{a}$ ordem de expansão na massa $m_{q}$, obtemos:

\section{Quarks Leves:}

$$
\begin{aligned}
S_{a b}^{(0)}(x) & =\left(\frac{i \not x}{2 \pi^{2} x^{4}}-\frac{m_{q}}{4 \pi^{2} x^{2}}\right) \delta_{a b} \\
S_{a b}^{(1)}(x) & =-\frac{i g_{s} \tau_{a b}^{n} G_{\mu \nu}^{n}(0)}{2^{5} \pi^{2} x^{2}}\left[\sigma^{\mu \nu} \not x+\not 火 \sigma^{\mu \nu}\right]
\end{aligned}
$$

Por outro lado, ao considerarmos o quark pesado $c$ - onde sua massa é da ordem de $1 \mathrm{GeV}$ - estas aproximações tornam-se impraticáveis. Logo, as expressões para os propagadores de quarks pesados, além de permanecerem no espaço dos momentos, são resolvidas de acordo com [44]:

\section{Quarks Pesados:}

$$
\begin{aligned}
& S_{a b}^{(0)}(p)=i \delta_{a b}\left(\frac{\not p+m_{c}}{p^{2}-m_{c}^{2}}\right) \\
& S_{a b}^{(1)}(p)=-\frac{i}{4} g_{s} \tau_{a b}^{n} G_{\mu \nu}^{n}(0)\left[\frac{\sigma^{\mu \nu} \not p+\not p \sigma^{\mu \nu}+2 m_{c} \sigma^{\mu \nu}}{\left(p^{2}-m_{c}^{2}\right)^{2}}\right]
\end{aligned}
$$

Convenientemente, para todo tipo de contribuição advinda de (2.12) mantemos a seguinte regra: propagadores de quarks leves devem ser descritos no espaço das coordenadas, enquanto que os propagadores de quarks pesados são descritos no espaço dos momentos. Nas seções seguintes analisaremos as contribuições não-perturbativas e as chamadas contribuições não-fatorizáveis, que irão acrescentar novidades à QCD perturbativa.

\subsubsection{Contribuições não-Perturbativas para os Quarks Leves}

Para os quarks leves $(u, d, s)$, estas contribuições têm sua origem no segundo termo de (2.12):

$$
\left\langle 0\left|: q_{a}(x) \bar{q}_{b}(0):\right| 0\right\rangle
$$

A OPE de Wilson consiste em transformar um produto não local de operadores, $q_{a}(x) \bar{q}_{b}(0)$, em uma soma de operadores locais $\hat{O}_{d}(0)$. Para isto, basta expandirmos em série de Taylor o operador $q_{a}(x)$, em torno de $x=0$ :

$$
q_{a}(x)=q_{a}(0)+\left.x_{\mu} \partial^{\mu} q_{a}(x)\right|_{x=0}+\left.\frac{1}{2} x_{\mu} x_{\nu} \partial^{\mu} \partial^{\nu} q_{a}(x)\right|_{x=0}+\cdots
$$

No gauge do ponto fixo: $x_{\mu} A^{\mu}=0$, as derivadas ordinárias dessa equação podem ser substituídas por derivadas covariantes.

$$
x_{\mu} \partial^{\mu} \longrightarrow x_{\mu} D^{\mu}
$$

onde $D_{\mu}=\partial_{\mu}-i g_{s} A_{\mu}$. Assim, o campo dos quarks torna-se:

$$
q_{a}(x)=q_{a}(0)+\left.x_{\mu} D^{\mu} q_{a}(x)\right|_{x=0}+\left.\frac{1}{2} x_{\mu} x_{\nu} D^{\mu} D^{\nu} q_{a}(x)\right|_{x=0}+\cdots
$$


Inserindo esta expansão em (2.23), obtemos:

$$
\left\langle 0\left|: q_{a}(x) \bar{q}_{b}(0):\right| 0\right\rangle=S_{a b}+x_{\mu} S_{a b}^{\mu}+\frac{1}{2} x_{\mu} x_{\nu} S_{a b}^{\mu \nu}+\cdots
$$

onde

$$
\begin{aligned}
S_{a b} & =\left\langle 0\left|: q_{a}(0) \bar{q}_{b}(0):\right| 0\right\rangle \\
S_{a b}^{\mu} & =\left\langle 0\left|: D^{\mu} q_{a}(x)\right|_{x=0} \bar{q}_{b}(0): \mid 0\right\rangle, \\
S_{a b}^{\mu \nu} & =\left\langle 0\left|: D^{\mu} D^{\nu} q_{a}(x)\right|_{x=0} \bar{q}_{b}(0): \mid 0\right\rangle .
\end{aligned}
$$

Logo, resolvendo cada termo da expressão (2.24) obteremos um tipo de contribuição nãoperturbativa.

(a) Condensado de Quarks: analisando o termo dominante da expansão (2.24), obtemos os condensados de quarks. Sua expressão é dada por:

$$
S_{a b}^{\alpha \beta}=\left\langle 0\left|: q_{a}^{\alpha}(0) \bar{q}_{b}^{\beta}(0):\right| 0\right\rangle=N \delta_{a b} \delta^{\alpha \beta}
$$

onde explicitamos os índices spinoriais $\alpha \beta$ e $N$ é um fator de normalização. Para determinar este fator, multiplicamos ambos os lados desta expressão por $\delta_{a b} \delta^{\alpha \beta}$ e reordenando os campos dos quarks obtemos:

$$
N=-\frac{1}{12}\langle 0|: \bar{q}(0) q(0):| 0\rangle \equiv-\frac{1}{12}\langle\bar{q} q\rangle
$$

portanto,

$$
S_{a b}=-\frac{\delta_{a b}}{12}\langle\bar{q} q\rangle
$$

onde $\langle\bar{q} q\rangle$ é o condensado de quarks e, usualmente, seu valor numérico é dado por [41]:

$$
\langle\bar{q} q\rangle=-(0.23 \pm 0.03)^{3} \mathrm{GeV}
$$

Podemos admitir correções na massa dos quarks que pertecem ao condensado. Para isto, basta considerar o segundo termo da expansão (2.24):

$$
S_{a b}^{\mu \alpha \beta}=\left\langle 0\left|: D^{\mu} q_{a}^{\alpha}(x)\right|_{x=0} \bar{q}_{b}^{\beta}(0): \mid 0\right\rangle=N \delta_{a b}\left(\gamma^{\mu}\right)^{\alpha \beta}
$$

multiplicando ambos os lados por $\delta_{a b}\left(\gamma_{\mu}\right)^{\beta \alpha}$, obtemos:

$$
N=-\frac{1}{48}\langle\bar{q} \not D q\rangle
$$

e usando a equação de $\operatorname{Dirac} \not D q_{a}^{\alpha}=-i m_{q} q_{a}^{\alpha}$, temos:

$$
N=i \frac{m_{q}}{48}\langle\bar{q} q\rangle
$$


Portanto,

$$
S_{a b}^{\mu}=i \frac{\delta_{a b} m_{q}}{48}\langle\bar{q} q\rangle \gamma^{\mu}
$$

e este termo não contribui no caso limite em que $m_{q} \rightarrow 0$.

(b) Condensado Misto: é importante ressaltar que esta contribuição vem, essencialmente, dos condensados mistos formados por quarks leves $(u, d, s)$. Considerando agora o terceiro termo da expansão (2.24):

$$
S_{a b}^{\mu \nu \alpha \beta}=\left\langle 0\left|: D^{\mu} D^{\nu} q_{a}^{\alpha}(x)\right|_{x=0} \bar{q}_{b}^{\beta}(0): \mid 0\right\rangle=N \delta_{a b} \delta^{\alpha \beta} g^{\mu \nu}
$$

multiplicando ambos os lados por $\delta_{a b} \delta^{\alpha \beta} g_{\mu \nu}$, obtemos:

$$
N=-\frac{1}{48}\left\langle\bar{q} D^{2} q\right\rangle
$$

Para calcular a expressão $D^{2} q$, podemos ver que da definição $D^{\mu}=\partial^{\mu}-i g_{s} A_{n}^{\mu} t^{n}$, podemos escrever

$$
\left[D^{\mu}, D^{\nu}\right]=-i g_{s} G^{\mu \nu}
$$

onde $G^{\mu \nu}=G_{n}^{\mu \nu} t^{n}$. Após algumas manipulações algébricas, é fácil demonstrar que

$$
\sigma_{\mu \nu}\left[D^{\mu}, D^{\nu}\right] q=2 i\left(\not D \not D-D^{2}\right) q=-i g_{s} \sigma \cdot G q
$$

mas $\not D \not D q=-m_{q}^{2} q$, então no caso de propagadores de quarks leves podemos desprezar a contribuição deste termo, visto que não contribui em $1^{\underline{a}}$ ordem em $m_{q}$. Logo

$$
D^{2} q=\frac{1}{2}\left(g_{s} \sigma \cdot G q\right) .
$$

Assim, o fator de normalização será dado por

$$
N=-\frac{1}{96}\left\langle\bar{q} g_{s} \sigma G q\right\rangle
$$

E, portanto, obtemos:

$$
S_{a b}^{\mu \nu}=-\frac{\delta_{a b} g^{\mu \nu}}{96}\left\langle\bar{q} g_{s} \sigma G q\right\rangle
$$

Usualmente, o condensado misto é expresso em termos do condensado de quarks através da relação:

$$
\left\langle\bar{q} g_{s} \sigma G q\right\rangle=m_{0}^{2}\langle\bar{q} q\rangle
$$

onde $m_{0}^{2}=0.8 \mathrm{GeV}^{2}[41]$.

Uma importante contribuição para os condensados mistos vem do quarto termo da expansão (2.24). Nele está presente a correção, em primeira ordem, para a massa do quark $m_{q}$. Sua expressão é dada por [44]:

$$
S_{a b}^{\mu \nu \rho}=\left\langle 0\left|: D^{\mu} D^{\nu} D^{\rho} q_{a}(x)\right|_{x=0} \bar{q}_{b}(0): \mid 0\right\rangle=\frac{i \delta_{a b} g^{\mu \nu} m_{q}}{3 \cdot 2^{6}}\left(\left\langle\bar{q} g_{s} \sigma G q\right\rangle\right) \gamma^{\rho}
$$


Embora possamos continuar a lidar com os demais termos da expansão (2.24), é preciso levar em conta a relevância e a complexidade que envolvem tais termos. Logo, sabendo que nas QCDSR os condensados de quarks, glúons e o misto representam a parte mais importante das contribuições dos condensados do vácuo, exploraremos neste trabalho apenas os termos proporcionais à eles. Ao inserir os termos (2.26), (2.29), (2.33) e (2.34) em (2.24), obtemos:

$$
\begin{aligned}
\left\langle 0\left|: q_{a}(x) \bar{q}_{b}(0):\right| 0\right\rangle=i \delta_{a b} & {\left[\left(\frac{\not x}{2 \pi^{2} x^{4}}+\frac{i m_{q}}{4 \pi^{2} x^{2}}\right)+\left(\frac{i}{12}+\frac{m_{q} \not x}{48}\right)\langle\bar{q} q\rangle+\right.} \\
& \left.+\left(\frac{i x^{2}}{3 \cdot 2^{6}}+\frac{m_{q} x^{2} \not x}{3^{2} 2^{7}}\right)\left\langle\bar{q} g_{s} \sigma G q\right\rangle\right]+\cdots
\end{aligned}
$$

\subsubsection{Contribuições não-Perturbativas para os Quarks Pesados}

Há ainda um efeito que está relacionado ao fato de que quarks emitem glúons. Nestes casos, para podermos continuar usando os propagadores nus dos quarks é necessário considerar que os glúons emitidos não possuem grandes momentos. Desta forma, os glúons irão interagir no regime de baixas energias e com isto obtemos os efeitos não-perturbativos. Estes efeitos são parametrizados pelos chamados condensados de glúons e somente são relevantes para os propagadores dos quarks pesados $(c)$ [40].

(a) Condensado de Glúons: Em diversos trabalhos [40, 41, 42] sobre QCDSR, foi verificado que os condensados de glúons desempenham um papel importante quando são formados por dois (ou mais) glúons emitidos de quarks pesados. Portanto, a contribuição nãoperturbativa dos condensados de glúons, de acordo com [44], é dada por:

$$
S_{a b}^{\left\langle G^{2}\right\rangle}(p)=\frac{i \delta_{a b} m_{c}\left\langle g_{s}^{2} G^{2}\right\rangle}{12\left(p^{2}-m_{c}^{2}\right)^{3}}\left[1+\frac{m_{c}\left(\not p+m_{c}\right)}{\left(p^{2}-m_{c}^{2}\right)}\right] .
$$

O condensado de glúons foi primeiramente estimado em análises de decaimento leptônico dos mésons, $\rho$ e $\phi$, e de análises do espectro do charmonium através das QCDSR. Seu valor numérico é estimado em [40, 41]:

$$
\left\langle g_{s}^{2} G^{2}\right\rangle=0.88 \mathrm{GeV}^{4}
$$

\subsubsection{Contribuições não-Fatorizáveis}

Para os quarks leves, ainda existe um outro tipo de contribuição a considerar na OPE. As chamadas contribuições não-fatorizáveis, surgem sempre que inserimos um glúon emitido por um quark externo $q^{\prime}$ nos propagadores (perturbativos ou não) de um quark $q$. Neste trabalho, o quark $q^{\prime}$ é simplesmente o quark pesado $c$. Estas contribuições são chamadas de não-fatorizáveis 
porque elas possuem índices, advindos do quark de origem $q^{\prime}$, que precisam ser contraídos com os do quark $q$. Existem dois tipos de condensados que devemos considerar:

(a) Condensado Misto Inserindo um glúon externo ao valor esperado no vácuo dos campos de quarks leves, temos a seguinte expressão:

$$
\left\langle 0\left|: q_{a}(x) g_{s} G_{\mu \nu}^{n} \bar{q}_{a}(0):\right| 0\right\rangle
$$

O fator $g_{s} G_{\mu \nu}^{n}$ aparece devido ao glúon emitido pelo quark externo $q^{\prime}$ e que interage com os campos do quark leve $q$. Se este glúon interagir no regime não-perturbativo, teremos de parametrizar os efeitos em termos do condensado misto. Para obter a expressão do operador local, façamos a expansão em série de Taylor do campo $q_{a}(x)$ em torno de $x=0$. Em primeira ordem, a equação (2.38) é dada por:

$$
\left\langle 0\left|: q_{a}^{\alpha}(0) g_{s} G_{\mu \nu}^{n} \bar{q}_{b}^{\beta}(0):\right| 0\right\rangle=N t_{a b}^{n}\left(\sigma_{\mu \nu}\right)^{\alpha \beta}
$$

multiplicando ambos os lados por $t_{b a}^{n}\left(\sigma_{\mu \nu}\right)^{\beta \alpha}$ e reordenado o condensado, obtemos:

$$
N=-\frac{1}{3 \cdot 2^{6}}\left\langle\bar{q} g_{s} \sigma G q\right\rangle
$$

portanto,

$$
\left\langle 0\left|: q_{a}(0) g_{s} G_{\mu \nu}^{n} \bar{q}_{b}(0):\right| 0\right\rangle=-\frac{t_{a b}^{n}}{3 \cdot 2^{6}}\left\langle\bar{q} g_{s} \sigma G q\right\rangle \sigma_{\mu \nu} .
$$

O termo de segunda ordem na expansão pode ser tratado de forma análoga e o resultado é dado por:

$$
\left\langle 0\left|: D^{\rho} q_{a}(x)\right|_{x=0} g_{s} G_{\mu \nu}^{n} \bar{q}_{b}(0): \mid 0\right\rangle=\frac{i m_{q} t_{a b}^{n}}{3 \cdot 2^{8}}\left\langle\bar{q} g_{s} \sigma G q\right\rangle\left(\gamma^{\rho} \sigma_{\mu \nu}+\sigma_{\mu \nu} \gamma^{\rho}\right)
$$

De tal forma que a equação (2.38), até segunda ordem de expansão, fica:

$$
\left\langle 0\left|: q_{a}(x) g_{s} G_{\mu \nu}^{n} \bar{q}_{b}(0):\right| 0\right\rangle=i t_{a b}^{n}\left[\frac{i \sigma_{\mu \nu}}{3 \cdot 2^{6}}+\frac{m_{q}}{3 \cdot 2^{8}}\left(\not \sigma_{\mu \nu}+\sigma_{\mu \nu} \not x\right)\right]\left\langle\bar{q} g_{s} \sigma G q\right\rangle \text {. }
$$

(b) Condensado de Glúons Há também um termo não-fatorizável para o condensado de glúon. Ele surge quando o glúon emitido por um quark leve $q$ interage, no regime de baixas energias, com um glúon emitido por um quark $q^{\prime}$. De acordo com [44], a expressão do propagador para este processo é dada por:

$$
\left.S_{a b}^{\left\langle G^{2}\right\rangle}(x)=-\frac{i t_{a b}^{n}}{3 \cdot 2^{9} \pi^{2} x^{2}}\left[\not \sigma_{\mu \nu}+\sigma_{\mu \nu} \not\right)\right]\left\langle g_{s}^{2} G^{2}\right\rangle .
$$

Obviamente, todas as expressões não-fatorizáveis deverão sempre estar associadas com a emissão de um glúon por um quark externo $q^{\prime}$. 


\subsubsection{Resumo das Contribuições da OPE}

Por fim, podemos expressar o propagador total (2.12) que contém as contribuições pertubativas, não-perturbativas e não-fatorizáveis, considerando os condensados de até dimensão 5 . Uma forma bastante útil de representá-lo é por meio de diagramas. Abaixo, seguem as expressões do propagador total para os quarks leves e pesados:

Quarks Leves: (espaço das coordenadas)

$$
\begin{aligned}
& S_{a b}^{q}(x)=\longrightarrow+\frac{x}{x}+\cdots+\cdots \cdot+\cdots \\
& +\longrightarrow \frac{\varepsilon^{3}}{\xi^{3}}+\longrightarrow \\
& S_{a b}^{q}(x)=i \delta_{a b}\left[\left(\frac{\not x}{2 \pi^{2} x^{4}}+\frac{i m_{q}}{4 \pi^{2} x^{2}}\right)+\left(\frac{i}{12}+\frac{m_{q} \not x}{32^{4}}\right)\langle\bar{q} q\rangle+\left(\frac{i x^{2}}{32^{6}}+\frac{m_{q} x^{2} \not x}{3^{2} 2^{7}}\right)\left\langle\bar{q} g_{s} \sigma G q\right\rangle\right]+ \\
& -i t_{a b}^{n}\left[\frac{\left(\not \partial \sigma_{\mu \nu}+\sigma_{\mu \nu} \not\right)}{32^{9} \pi^{2} x^{2}}\right]\left\langle g_{s}^{2} G^{2}\right\rangle+i t_{a b}^{n}\left[\frac{i \sigma_{\mu \nu}}{32^{6}}+\frac{m_{q}}{32^{8}}\left(\not \sigma_{\mu \nu}+\sigma_{\mu \nu} \not\right)\right]\left\langle\bar{q} g_{s} \sigma G q\right\rangle
\end{aligned}
$$

Quarks Pesados: (espaço dos momentos)

$$
\begin{gathered}
S_{a b}^{c}(p)=\longrightarrow+\longrightarrow \frac{\vec{c}}{\longrightarrow}+\longrightarrow \frac{i t_{a b}^{n}}{4\left(p^{2}-m_{c}^{2}\right)^{2}}\left[\frac{\sigma^{\mu \nu} \not p+\not p \sigma^{\mu \nu}+2 m_{c} \sigma^{\mu \nu}}{\left(p^{2}-m_{c}^{2}\right)^{2}}\right]+ \\
S_{a b}^{c}(p)=\frac{i \delta_{a b}}{p^{2}-m_{c}^{2}}\left(\not p+m_{c}\right)-\frac{i \delta_{a b} m_{c}\left\langle g_{s}^{2} G^{2}\right\rangle}{32^{2}\left(p^{2}-m_{c}^{2}\right)^{3}}\left[1+\frac{m_{c}\left(\not p+m_{c}\right)}{\left(p^{2}-m_{c}^{2}\right)}\right]
\end{gathered}
$$


Uma vez calculada a OPE até a dimensão desejada e obtida a expressão analítica de (2.1), o próximo passo é escrevê-la na forma de uma relação de dispersão:

$$
\Pi^{o p e}\left(q^{2}\right)=\frac{1}{\pi} \int_{s_{\min }}^{\infty} d s \frac{\operatorname{Im}[\Pi(s)]}{s-q^{2}}+\cdots \equiv \int_{s_{\min }}^{\infty} d s \frac{\rho^{o p e}(s)}{s-q^{2}}+\cdots
$$

onde $\cdots$ são termos de subtração e

$$
\rho^{\text {ope }}(s)=\frac{1}{\pi} \operatorname{Im}[\Pi(s)]
$$

é a densidade espectral no lado da OPE.

A vantagem deste formalismo é possibilitar uma melhor comparação do lado da QCD com o lado fenomenológico e também poder separar as contribuições do pólo e do contínuo.

\subsection{Lado Fenomenológico}

A função de correlação (2.1) também pode ser avaliada em termos de hádrons. Para isto, considere novamente o tensor de polarização

$$
\Pi_{\mu \nu}(q)=i \int d^{4} x e^{i q \cdot x}\left\langle 0\left|T\left[j_{\mu}(x) j_{\nu}^{\dagger}(0)\right]\right| 0\right\rangle \equiv \int d^{4} x e^{i q \cdot x} \Pi_{\mu \nu}(x)
$$

onde definimos $\Pi_{\mu \nu}(x)=i\left\langle 0\left|T\left[j_{\mu}(x) j_{\nu}^{\dagger}(0)\right]\right| 0\right\rangle$. Assim, vemos que $\Pi_{\mu \nu}(x)$ é a Transformada de Fourier de $\Pi_{\mu \nu}(q)$.

Inserindo um conjunto completo de estados hadrônicos entre as correntes vetoriais:

$$
\sum_{H} \frac{1}{(2 \pi)^{3}} \int \frac{d^{3} \vec{q}}{2 q_{0}}|H(q)\rangle\langle H(q)|=\hat{1}
$$

onde a soma envolve o estado fundamental e todas as ressonâncias do hádron estudado. Utilizando os operadores de translação definidos por:

$$
j_{\mu}(x)=e^{i q \cdot x} j_{\mu}(0) e^{-i q \cdot x}
$$

No que segue, substituíremos $j_{\mu}(0)$ por $j_{\mu}$. Da definição do produto temporalmente ordenado, obtemos:

$$
\Pi_{\mu \nu}(x)=i \sum_{H} \frac{1}{(2 \pi)^{3}} \int \frac{d^{3} \vec{q}}{2 q_{0}}\left[e^{-i q \cdot x} \theta\left(x_{0}\right)+e^{i q \cdot x} \theta\left(-x_{0}\right)\right]\left\langle 0\left|j_{\mu}\right| H(q)\right\rangle\left\langle H(q)\left|j_{\nu}^{\dagger}\right| 0\right\rangle
$$

Usando a relação

$$
\frac{1}{(2 \pi)^{3}} \int \frac{d^{3} \vec{q}}{2 q_{0}}\left[e^{-i q \cdot x} \theta\left(x_{0}\right)+e^{i q \cdot x} \theta\left(-x_{0}\right)\right] F(q)=i \int \frac{d^{4} q}{(2 \pi)^{4}} \frac{e^{-i q \cdot x}}{q^{2}-m^{2}+i \epsilon} F(q)
$$


a equação (2.50) pode ser reescrita como:

$$
\Pi_{\mu \nu}(x)=-\sum_{H} \int \frac{d^{4} q}{(2 \pi)^{4}} \frac{e^{-i q \cdot x}}{q^{2}-m^{2}}\left\langle 0\left|j_{\mu}\right| H(q)\right\rangle\left\langle H(q)\left|j_{\nu}^{\dagger}\right| 0\right\rangle
$$

que é a transformada inversa de Fourier com a função de correlação, no espaço dos momentos, dada por:

$$
\Pi_{\mu \nu}(q)=-\sum_{H} \frac{1}{q^{2}-m^{2}}\left\langle 0\left|j_{\mu}\right| H(q)\right\rangle\left\langle H(q)\left|j_{\nu}^{\dagger}\right| 0\right\rangle
$$

Contraindo os índices de Lorentz, obtemos a função de correlação fenomenológica $\Pi^{\text {fen }}(q)$ :

$$
\Pi^{f e n}(q) \equiv \Pi_{\mu}^{\mu}(q)=-\sum_{H} \frac{1}{\left(q^{2}-m^{2}\right)}\left\langle 0\left|j_{\mu}\right| H(q)\right\rangle\left\langle H(q)\left|j^{\dagger \mu}\right| 0\right\rangle
$$

Em geral, o acoplamento do estado fundamental de um hádron vetorial $H_{0}(q)$ à uma corrente $j_{\mu}$ é parametrizado por:

$$
\left\langle 0\left|j_{\mu}\right| H_{0}(q)\right\rangle=\lambda m \epsilon_{\mu}
$$

onde $\lambda$ é um parâmetro fenomenológico associado à constante de decaimento do hádron e $\epsilon_{\mu}$ é o vetor de polarização. Logo, separando o estado fundamental dos estados ressonantes em (2.54) e inserindo o acoplamento (2.55), obtemos:

$$
\Pi^{f e n}(q)=-\frac{\lambda^{2} m^{2}}{\left(q^{2}-m^{2}\right)} \sum_{p o l} \epsilon^{\mu} \epsilon_{\mu}-\sum_{H_{i}} \frac{1}{\left(q^{2}-m^{2}\right)}\left\langle 0\left|j_{\mu}\right| H_{i}(q)\right\rangle\left\langle H_{i}(q)\left|j^{\dagger \mu}\right| 0\right\rangle
$$

onde $H_{i}(q)$ representa os estados ressonantes do hádron e $\sum_{p o l} \epsilon_{\mu} \epsilon_{\nu}=\left(-g_{\mu \nu}+\frac{q_{\mu} q_{\nu}}{m^{2}}\right)$, logo:

$$
\Pi^{f e n}(q)=\lambda^{2} m^{2}\left[\frac{3 q^{2}}{m^{2}\left(q^{2}-m^{2}\right)}-\frac{4}{m^{2}}\right]-\sum_{H_{i}} \frac{1}{\left(q^{2}-m^{2}\right)}\left\langle 0\left|j_{\mu}\right| H_{i}(q)\right\rangle\left\langle H_{i}(q)\left|j^{\dagger \mu}\right| 0\right\rangle
$$

Provaremos adiante, que ao aplicarmos a Transformada de Borel em termos constantes o resultado será nulo. Por esta razão, eliminamos o segundo termo do estado fundamental, e o resultado obtido para a função de correlação fenomenológica é:

$$
\Pi^{f e n}(q)=\frac{3 \lambda^{2} q^{2}}{q^{2}-m^{2}}-\sum_{H_{i}} \frac{1}{\left(q^{2}-m^{2}\right)}\left\langle 0\left|j_{\mu}\right| H_{i}(q)\right\rangle\left\langle H_{i}(q)\left|j^{\dagger \mu}\right| 0\right\rangle
$$

Utilizando a relação (2.3), obtemos a função analítica $\Pi^{f e n}\left(q^{2}\right)$ a partir da função de correlação:

$$
\Pi^{f e n}\left(q^{2}\right)=\frac{\lambda^{2}}{m^{2}-q^{2}}+\sum_{H_{i}} \frac{1}{3 q^{2}\left(q^{2}-m^{2}\right)}\left\langle 0\left|j_{\mu}\right| H_{i}(q)\right\rangle\left\langle H_{i}(q)\left|j^{\dagger \mu}\right| 0\right\rangle
$$

\subsection{Relação de Dispersão}

Na seção anterior, vimos como a função de correlação pode ser descrita tanto em termos de quarks (Lado da QCD) como em termos de hádrons (Lado Fenomenológico). Muitas vezes, as 
funções de correlação apresentam valores reais para $q^{2}$ abaixo de algum valor limite qualquer (que chamamos convenientemente de $s_{\text {min }}$ ) e exibem um corte de ramificação para $q^{2}$ maior que tal limite. Formalmente, é possível tratar as funções de correlação como funções analíticas complexas.

Dada uma função complexa, $\Pi(z)$, considere os seguintes pontos:

- $\Pi(z)$ é real para valores reais de $z$, tal que $z<s_{\text {min }}$;

- $\Pi(z)$ tem cortes de ramificação para valores reais de $z$, quando $z>s_{\min }$;

- $\Pi(z)$ é analítica para valores complexos de $z$ (exceto ao longo dos cortes de ramificação).

É comum representar $\Pi(z)$ no plano complexo através da figura (2.4).

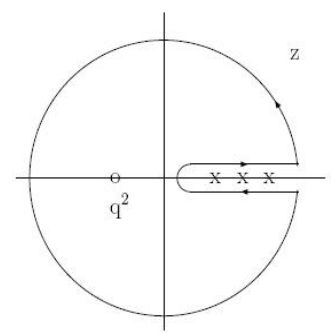

Figura 2.4: O contorno escolhido no plano complexo da variável $z=q^{2}$. O ponto aberto corresponde ao estado fundamental, para $q^{2}<0$. E as posições dos estados ressonantes, para $q^{2}>0$, são indicadas pelas cruzes [42].

Logo, empregando a fórmula de Cauchy para a função analítica $\Pi(z)$, em que $z=q^{2}$, e escolhendo o contorno indicado, obtemos:

$$
\begin{aligned}
\Pi\left(q^{2}\right) & =\frac{1}{2 \pi i} \oint_{C} d z \frac{\Pi(z)}{z-q^{2}} \\
& =\frac{1}{2 \pi i} \oint_{|z|=R} d z \frac{\Pi(z)}{z-q^{2}}+\frac{1}{2 \pi i} \int_{0}^{R} d z \frac{\Pi(z+i \epsilon)-\Pi(z-i \epsilon)}{z-q^{2}} .
\end{aligned}
$$

Fazendo $R \rightarrow \infty$ e admitindo que a função $\Pi(z)$ vai a zero no infinito, então a primeira integral desaparece. Para resolver a segunda integral, utilizamos o princípio de reflexão de Schwartz [42]:

$$
\Pi(z+i \epsilon)-\Pi(z-i \epsilon)=2 i \operatorname{Im}[\Pi(z)] \quad, \text { tal que } z>s_{\min }
$$

Inserindo este resultado em (2.60), obtemos a relação de dispersão:

$$
\Pi\left(q^{2}\right)=\frac{1}{\pi} \int_{s_{\min }}^{\infty} d s \frac{\operatorname{Im}[\Pi(s)]}{s-q^{2}-i \epsilon} \equiv \int_{s_{\min }}^{\infty} d s \frac{\rho(s)}{s-q^{2}-i \epsilon}
$$

onde fizemos a troca de variável $z=s$ e

$$
\rho(s)=\frac{1}{\pi} \operatorname{Im}[\Pi(s)]
$$


é a densidade espectral fenomenológica.

Usualmente, a densidade espectral $\rho(s)$ pode ser descrita por um pólo bem definido, representado pela partícula no estado fundamental e mais um contínuo, representado pelo seus estados ressonantes, de tal forma que:

$$
\rho(s)=\lambda^{2} \delta\left(s-m^{2}\right)+\rho^{c o n t}(s)
$$

Fenomenologicamente, só há contribuição das ressonâncias quando estamos acima de um valor limiar (ou threshold) do contínuo, indicado por $s_{0}$. Parametrizamos então $\rho^{\text {cont }}$ na forma [45]:

$$
\rho^{\text {cont }}(s)=\rho^{\text {ope }}(s) \Theta\left(s-s_{0}\right),
$$

$\operatorname{com} \rho^{\text {ope }}(s)$ dado por (2.46). Portanto,

$$
\rho(s)=\lambda^{2} \delta\left(s-m^{2}\right)+\rho^{o p e}(s) \Theta\left(s-s_{0}\right)
$$

Inserindo (2.65) em (2.61), obtemos:

$$
\Pi\left(q^{2}\right)=\frac{\lambda^{2}}{m^{2}-q^{2}}+\int_{s_{0}}^{\infty} d s \frac{\rho^{\text {ope }}(s)}{s-q^{2}}
$$

Note que o primeiro termo desta equação é exatamente igual ao primeiro termo de (2.59). Com isto, obtemos uma expressão mais elegante para as contribuições dos estados ressonantes:

$$
\sum_{H_{i}} \frac{1}{3 q^{2}\left(q^{2}-m^{2}\right)}\left\langle 0\left|j_{\mu}\right| H_{i}(q)\right\rangle\left\langle H_{i}(q)\left|j^{\dagger \mu}\right| 0\right\rangle \equiv \int_{s_{0}}^{\infty} d s \frac{\rho^{o p e}(s)}{s-q^{2}}
$$

De fato, (2.66) é a que será utilizada na comparação de ambos os lados da teoria.

\subsection{Transformada de Borel}

Embora a comparação dos lados da QCD e Fenomenológico seja garantida pelo Princípio da Dualidade Quark-Hádron, o uso das aproximações que fizemos em ambos os lados introduzem algumas complicações. No lado da QCD, ao lidarmos com contribuições não-perturbativas, à rigor, deveríamos calcular os infinitos termos da OPE. Contudo, a impraticabilidade deste procedimento nos leva a utilizar técnicas que garantam o truncamento da série e permitam calcular grandezas físicas com um certo grau de confiança. No lado Fenomenológico, a aproximação (2.64) nos permite calcular a contribuição do estado fundamental do hádron isoladamente da contribuição do contínuo. Essas são as dificuldades a serem resolvidas no método das QCDSR.

Uma maneira prática de suprimir as contribuições de ordem mais alta da OPE é o uso da Transformada de Borel, cuja definição é dada por [42]:

$$
\mathcal{B}\left[\Pi\left(Q^{2}\right)\right] \equiv \hat{\Pi}\left(M^{2}\right)=\lim _{\substack{Q^{2}, n \rightarrow \infty \\ Q^{2} / n=M^{2}}} \frac{\left(Q^{2}\right)^{n+1}}{n !}\left(\frac{\partial}{\partial Q^{2}}\right)^{n} \Pi\left(Q^{2}\right)
$$


onde $Q^{2}$ é o momento da partícula no espaço euclideano $\left(Q^{2}=-q^{2}\right)$. O parâmetro $M$ é chamado massa de Borel. Alguns importantes exemplos de transformadas são:

$$
\begin{aligned}
\mathcal{B}\left[\left(Q^{2}\right)^{k}\right] & = \begin{cases}0 & \text { se } k \geq 0 ; \\
\frac{1}{(k-1) !}\left(\frac{1}{M^{2}}\right)^{k-1} & \text { se } k<0 .\end{cases} \\
\mathcal{B}\left[\frac{1}{s+Q^{2}}\right] & =e^{-s / M^{2}} .
\end{aligned}
$$

A transformada de Borel ajuda a convergência da OPE suprimindo a contribuição dos operadores de dimensão mais alta. Os coeficientes destes operadores são proporcionais às potências negativas de $\left(Q^{2}\right)^{k}$, onde $k$ cresce com a dimensão do operador. Logo, segundo as transformadas de Borel acima, a inserção do fator $1 /(k-1)$ ! reduz a importância dos operadores de ordem superior.

Do lado Fenomenológico, a função de correlação assume a forma geral:

$$
\Pi\left(Q^{2}\right)=\sum_{i=0}^{\infty} \frac{K_{i}}{m_{i}^{2}+Q^{2}}
$$

onde $m_{0}$ é massa do hádron no estado fundamental, $m_{i}$ as massas dos estados ressonantes e $K_{i}$ são constantes. Usando a equação (2.70), temos:

$$
\mathcal{B}\left[\Pi\left(Q^{2}\right)\right]=\sum_{i=0}^{\infty} K_{i} e^{-m_{i}^{2} / M^{2}} .
$$

Como $m_{i+1}>m_{i}$ a transformada de Borel suprime os estados ressonantes.

Logo, se for possível encontrar uma região, no espaço da massa de Borel, em que as contribuições dos operadores de ordem mais altas da OPE e as ressonâncias de massas mais altas sejam igualmente suprimidas, as descrições hadrônica e da QCD se igualarão e poderemos extrair as propriedades hadrônicas de interesse para o estado fundamental dos hádrons envolvidos. Então, devemos determinar um intervalo em que a massa de Borel seja adequada para a efetiva comparação do lado da QCD e do lado Fenomenológico. Este intervalo é geralmente chamado de Janela de Borel.

\subsubsection{Janela de Borel}

Para determinarmos uma Janela de Borel, precisamos analisar os seguintes pontos:

\section{Dominância do Pólo sobre o Contínuo}

Como estamos interessados em estudar o estado fundamental dos hádrons, devemos trabalhar numa região de $M^{2}$ onde a contribuição do pólo seja maior que a contribuição do contínuo. Dessa forma, tentamos garantir que as contribuições que dão origem à massa, venham diretamente do estado fundamental. Como vimos, os estados ressonantes começam a surgir a partir de um limiar do contínuo, $s_{0}$. Logo, da equação (2.75) temos separadamente as contribuições do pólo (P) e as 
do contínuo (C) e a contribuição total (T) é dada pela soma de ambas. Desta forma, temos:

$$
\begin{aligned}
& \mathrm{P}=\frac{\lambda^{2}}{m^{2}-q^{2}} \\
& \mathrm{C}=\int_{s_{0}}^{\infty} d s \frac{\rho^{o p e}(s)}{s-q^{2}} \\
& \mathrm{~T}=\mathrm{P}+\mathrm{C} .
\end{aligned}
$$

Para valores baixos da massa de Borel, a contribuição de C é muito pequena, de forma que temos a condição $\mathrm{P}>\mathrm{C}$. Porém, a medida que aumentamos o valor de $M^{2}$, C vai se tornando cada vez mais importante e para um dado valor de $M^{2}$ temos que a contribuição P e C são iguais. E é neste ponto que definimos o limite superior para a massa de Borel, $M_{\max }^{2}$.

\section{Convergência da OPE}

A transformada de Borel faz com que termos que antes dependiam de potências cada vez mais altas de $1 / q^{2}$, agora dependam de potências crescentes do parâmetro livre $1 / M^{2}$. Com isto, conforme aumentamos o valor de $M^{2}$ os termos superiores da OPE tendem a contribuir menos, melhorando a convergência da OPE. Logo, é possível identificar o valor de $M^{2}$ no qual a série passa a convergir fornecendo o limite inferior da massa de Borel, $M_{m i n}^{2}$. Portanto, se a seguinte condição for satisfeita: $M_{\text {min }}^{2}<M_{\text {max }}^{2}$, então teremos uma região em que os resultados das QCDSR são confiáveis. Esta região é a chamada Janela de Borel. Caso, não exista esta região, os resultados obtidos não são confiáveis. Na maioria dos casos, a convergência da OPE já é obtida com a contribuição dos condensados com dimensão até 6 , bastando apenas analisar se os valores de $M_{\text {min }}^{2}$ e $M_{\text {max }}^{2}$, são suficientes para garantir uma Janela de Borel para as QCDSR.

\section{Estabilidade de $M^{2}$}

Espera-se que a massa do hádron possua uma certa estabilidade em seu valor, dentro da Janela de Borel. Isto porque, teoricamente a massa independe do parâmetro $M^{2}$. Portanto, quando há uma grande dependência de $m$ com relação à $M^{2}$, esta é uma forte indicação de que devemos reavaliar as QCDSR, ou incluindo mais condensados ou considerando outras aproximações.

\subsection{Princípio da Dualidade Quark-Hádron}

Aplicando a Transformada de Borel em (2.45) e (2.66) obtemos, respectivamente:

$$
\Pi^{o p e}\left(M^{2}\right)=\int_{s_{\text {min }}}^{\infty} d s \rho^{o p e}(s) e^{-s / M^{2}}
$$

e

$$
\Pi^{f e n}\left(M^{2}\right)=\lambda^{2} e^{-m^{2} / M^{2}}+\int_{s_{0}}^{\infty} d s \rho^{o p e}(s) e^{-s / M^{2}}
$$


Assim, com a supressão dos operadores de dimensão mais altas no lado da QCD e a supressão das constribuições dos estados ressonantes no lado Fenomenológico, as funções de correlação (2.74) e (2.75) podem ser comparadas:

$$
\lambda^{2} e^{-m^{2} / M^{2}}+\int_{s_{0}}^{\infty} d s \rho^{o p e}(s) e^{-s / M^{2}}=\int_{s_{\min }}^{\infty} d s \rho^{o p e}(s) e^{-s / M^{2}}
$$

considerando que $\rho^{o p e}(s)$ é contínuo em todo o intervalo de integração, então

$$
\begin{aligned}
\lambda^{2} e^{-m^{2} / M^{2}}+\int_{s_{0}}^{\infty} d s \rho \rho p e(s) e^{-s / M^{2}} & =\int_{s_{\min }}^{s_{0}} d s \rho^{\text {ope }}(s) e^{-s / M^{2}}+\int_{s_{0}}^{\infty} d s \rho \rho p e(s) e^{-s / M^{2}} \\
\lambda^{2} e^{-m^{2} / M^{2}} & =\int_{s_{\min }}^{s_{0}} d s \rho^{\text {ope }}(s) e^{-s / M^{2}}
\end{aligned}
$$

Derivando (2.76) com relação ao termo $1 / M^{2}$, temos:

$$
\lambda^{2} m^{2} e^{-m^{2} / M^{2}}=\int_{s_{\min }}^{s_{0}} d s s \rho^{o p e}(s) e^{-s / M^{2}} .
$$

Dividindo (2.77) por (2.76), obtemos:

$$
m^{2}=\frac{\int_{s_{\min }}^{s_{0}} d s s \rho^{o p e}(s) e^{-s / M^{2}}}{\int_{s_{\min }}^{s_{0}} d s \rho^{o p e}(s) e^{-s / M^{2}}}
$$

que é a equação das QCDSR que determina a massa do hádron estudado. 


\section{Capítulo 3}

\section{Família de Mésons Y em QCDSR}

As partículas que queremos analisar foram observadas nos seguintes processos:

$$
\begin{array}{cl}
\operatorname{BaBar}[\mathbf{1 0 ]}: & e^{+} e^{-} \longrightarrow Y(4260) \longrightarrow \gamma_{I S R} J / \psi \pi^{+} \pi^{-} \\
\text {Belle [11]: } & e^{+} e^{-} \longrightarrow Y(4360) \longrightarrow \gamma_{I S R} \psi^{\prime} \pi^{+} \pi^{-} \\
& e^{+} e^{-} \longrightarrow Y(4660) \longrightarrow \gamma_{I S R} \psi^{\prime} \pi^{+} \pi^{-}
\end{array}
$$

Entre parênteses é indicada as suas respectivas massas, em MeV. Como discutido na Introdução, os estados intermediários: $Y(4260), Y(4360)$ e $Y(4660)$ possuem números quânticos dados por $J^{P C}=1^{--}$e em seus canais de decaimento há a presença do charmonium $J / \psi$ ou de seu estado ressonante $\psi^{\prime}$. Portanto, o conteúdo mínimo de quarks desses estados é $c \bar{c}$. Como tais estados não se ajustam ao espectro do charmonium, uma nova análise acerca de suas estruturas faz-se necessária.

Neste trabalho, construiremos estados que possam ser descritos por estados de tetraquarks: $[c q \bar{c} \bar{q}]$ e $[c s \bar{c} \bar{s}]$; ou por estados moleculares: $D_{s 0} \bar{D}_{s}^{*}$ e $D_{0} \bar{D}^{*}$. Através das QCDSR, calculamos as suas respectivas massas e comparamos com os valores experimentais das massas dos mésons $Y(4260), Y(4360)$ e $Y(4660)$.

O primeiro passo das QCDSR é a construção correta da corrente mesônica a ser inserida em (2.1). É na corrente que inserimos a suposição acerca da estrutura interna da partícula estudada (tetraquark ou molécula) e também seus números quânticos.

\subsection{Os Estados de Tetraquarks}

Uma possível corrente que descreve um estado de tetraquark com distribuição simétrica do spin: $[c q]_{S=0}[\bar{c} \bar{q}]_{S=1}+[c q]_{S=1}[\bar{c} \bar{q}]_{S=0}$, é dada por:

$$
j_{\mu}=\frac{\epsilon_{a b c} \epsilon_{d e c}}{\sqrt{2}}\left[\left(q_{a}^{T} C \gamma_{5} c_{b}\right)\left(\bar{q}_{d} \gamma_{\mu} \gamma_{5} C \bar{c}_{e}^{T}\right)+\left(q_{a}^{T} C \gamma_{5} \gamma_{\mu} c_{b}\right)\left(\bar{q}_{d} \gamma_{5} C \bar{c}_{e}^{T}\right)\right]
$$

onde $a, b, c, \ldots$ são índices de cor, $\gamma_{\mu}$ as matrizes de Dirac, $q$ e $c$ são os campos fermiônicos dos quarks: $q=$ (up, down, strange) e $c=$ charm; $C$ é a matriz de conjugação de carga; e $\epsilon_{a b c}$ é um tensor totalmente antissimétrico. 


\subsubsection{Transformações de Paridade e Conjugação de Carga}

Antes de prosseguirmos, é necessário verificar que (3.1) possui os números quânticos dos mésons vetoriais Y. Para isto, consideremos as seguintes transformações para os campos fermiônicos, $\psi$, dos quarks:

$$
\begin{aligned}
\mathcal{P} \psi \mathcal{P}^{-1} & =\gamma_{0} \psi \\
\mathcal{C} \psi \mathcal{C}^{-1} & =C \bar{\psi}^{T}
\end{aligned}
$$

onde $\mathcal{P}$ é o operador paridade e $\mathcal{C}$ é o operador de conjugação de carga.

Logo, para calcular a paridade da corrente (3.1), inserimos as transformações (3.2) nos campos de quarks contidos nela:

$$
\begin{aligned}
\mathcal{P} j_{\mu} \mathcal{P}^{-1} & =\frac{\epsilon_{a b c} \epsilon_{d e c}}{\sqrt{2}}\left[\left(q_{a}^{T} \gamma_{0}^{T} C \gamma_{5} \gamma_{0} c_{b}\right)\left(\bar{q}_{d} \gamma_{0} \gamma_{\mu} \gamma_{5} C \gamma_{0}^{T} \bar{c}_{e}^{T}\right)+\left(q_{a}^{T} \gamma_{0}^{T} C \gamma_{5} \gamma_{\mu} \gamma_{0} c_{b}\right)\left(\bar{q}_{d} \gamma_{0} \gamma_{5} C \gamma_{0}^{T} \bar{c}_{e}^{T}\right)\right] \\
& =\frac{\epsilon_{a b c} \epsilon_{d e c}}{\sqrt{2}}\left[\left(q_{a}^{T} C \gamma_{0}^{T} \gamma_{0} \gamma_{5} c_{b}\right)\left(\bar{q}_{d} \gamma_{0} \gamma_{\mu} \gamma_{0}^{T} \gamma_{5} C \bar{c}_{e}^{T}\right)+\left(q_{a}^{T} C \gamma_{5} \gamma_{0}^{T} \gamma_{\mu} \gamma_{0} c_{b}\right)\left(\bar{q}_{d} \gamma_{0} \gamma_{0}^{T} \gamma_{5} C \bar{c}_{e}^{T}\right)\right] \\
& =\frac{\epsilon_{a b c} \epsilon_{d e c}}{\sqrt{2}}\left[\left(q_{a}^{T} C \gamma_{5} c_{b}\right)\left(\bar{q}_{d} \gamma_{\mu}^{\dagger} \gamma_{5} C \bar{c}_{e}^{T}\right)+\left(q_{a}^{T} C \gamma_{5} \gamma_{\mu}^{\dagger} c_{b}\right)\left(\bar{q}_{d} \gamma_{5} C \bar{c}_{e}^{T}\right)\right]
\end{aligned}
$$

Para o desenvolvimento de (3.3), foram utilizadas as relações (B.7), (B.12), (B.13) e (B.19). Sabendo que as matrizes de Dirac satisfazem as seguintes relações:

$$
\begin{aligned}
& \gamma_{0}=\gamma_{0}^{\dagger} \\
& \gamma_{i}=-\gamma_{i}^{\dagger}
\end{aligned}
$$

onde $i=1,2,3$. Então:

$$
\begin{aligned}
& \mathcal{P} j_{0} \mathcal{P}^{-1}=j_{0} \\
& \mathcal{P} j_{i} \mathcal{P}^{-1}=-j_{i} .
\end{aligned}
$$

Portanto, concluímos que a corrente (3.1) é ímpar por transformações de paridade.

Resta agora determinarmos a conjugação de carga de (3.1):

$$
\begin{aligned}
\mathcal{C} j_{\mu} \mathcal{C}^{-1}= & \frac{\epsilon_{a b c} \epsilon_{d e c}}{\sqrt{2}}\left[\left(\bar{q}_{a} C^{-1} C \gamma_{5} C \bar{c}_{b}^{T}\right)\left(\left(-q_{d}^{T} C^{-1}\right) \gamma_{\mu} \gamma_{5} C\left(-C c_{e}\right)\right)+\right. \\
& \left.+\left(\bar{q}_{a} C^{-1} C \gamma_{5} \gamma_{\mu} C \bar{c}_{b}^{T}\right)\left(\left(-q_{d}^{T} C^{-1}\right) \gamma_{5} C\left(-C c_{e}\right)\right)\right] \\
= & \frac{\epsilon_{a b c} \epsilon_{d e c}}{\sqrt{2}}\left[\left(\bar{q}_{a} \gamma_{5} C \bar{c}_{b}^{T}\right)\left(q_{d}^{T} C \gamma_{\mu} \gamma_{5} c_{e}\right)+\left(\bar{q}_{a} \gamma_{5} \gamma_{\mu} C \bar{c}_{b}^{T}\right)\left(q_{d}^{T} C \gamma_{5} c_{e}\right)\right] .
\end{aligned}
$$

Note que, fazendo a troca de índices: $a \leftrightarrow d$ e $b \leftrightarrow e$; e utilizando as relações $\left[C, \gamma_{5}\right]=0$ e $\left\{\gamma_{\mu}, \gamma_{5}\right\}=0$, a equação (3.5) torna-se:

$$
\begin{aligned}
\mathcal{C} j_{\mu} \mathcal{C}^{-1} & =\frac{\epsilon_{a b c} \epsilon_{d e c}}{\sqrt{2}}\left[-\left(q_{a}^{T} C \gamma_{5} c_{b}\right)\left(\bar{q}_{d} \gamma_{\mu} \gamma_{5} C \bar{c}_{e}^{T}\right)-\left(q_{a}^{T} C \gamma_{5} \gamma_{\mu} c_{b}\right)\left(\bar{q}_{d} \gamma_{5} C \bar{c}_{e}^{T}\right)\right] \\
& =-j_{\mu} .
\end{aligned}
$$


Isto demonstra que a corrente (3.1) é ímpar por conjugação de carga.

Fica provado, portanto, que os números quânticos que a corrente (3.1) reproduz são dados por $J^{P C}=1^{--}$, que são os mesmos números quânticos da família de mésons $Y$.

\subsubsection{Lado Fenomenológico}

Para construir o lado fenomenológico das regras de soma nós parametrizamos o acoplamento do méson vetorial $Y$ com a corrente na Eq.(3.1) através da relação:

$$
\left\langle 0\left|j_{\mu}\right| Y\right\rangle=f_{Y} m_{Y}^{4} \epsilon_{\mu} \equiv \lambda \epsilon_{\mu}
$$

onde $f_{Y}$ é a constante de decaimento do méson e definimos $\lambda=f_{Y} m_{Y}^{4}$.

Assim, a função de correlação no lado fenomenológico (2.75) pode ser reescrita como:

$$
\Pi^{f e n}\left(q^{2}\right)=f_{Y}^{2} m_{Y}^{6} e^{-m^{2} / M^{2}}+\int_{s_{0}}^{\infty} d s \rho^{o p e}(s) e^{-m^{2} / M^{2}} .
$$

Logo, resta calcular a função de correlação no lado da QCD.

\subsubsection{Lado da QCD}

A função de correlação de dois pontos, no caso da corrente vetorial, é dada por:

$$
\Pi_{\mu \nu}(q)=i \int d^{4} x e^{i q \cdot x}\left\langle 0\left|T\left[j_{\mu}(x) j_{\nu}^{\dagger}(0)\right]\right| 0\right\rangle .
$$

Como a corrente vetorial é conservada, a Eq. (3.9) pode ser reescrita como:

$$
\Pi_{\mu \nu}(q)=-\Pi\left(q^{2}\right)\left(g_{\mu \nu} q^{2}-q_{\mu} q_{\nu}\right)
$$

de onde obtemos

$$
\Pi_{\mu}^{\mu}(q)=-3 q^{2} \Pi\left(q^{2}\right)
$$

Inserindo a corrente mesônica, Eq. (3.1), na função de correlação (3.9), obtemos:

$$
\begin{aligned}
& \Pi\left(q^{2}\right)=-\frac{i \epsilon_{a b c} \epsilon_{d e c} \epsilon_{i j k} \epsilon_{l m k}}{6 q^{2}} \int d^{4} x e^{i q \cdot x} \\
& \times\left\{\begin{array}{rlc} 
& \operatorname{Tr}\left[S_{b i}^{c}(x) \gamma_{5} C S_{a j}^{q^{T}}(x) C \gamma_{5}\right] \cdot \operatorname{Tr}\left[S_{m d}^{q}(-x) \gamma_{\mu} \gamma_{5} C S_{l e}^{c^{T}}(-x) C \gamma_{5} \gamma^{\mu}\right] & + \\
+ & \operatorname{Tr}\left[S_{b i}^{c}(x) \gamma^{\mu} \gamma_{5} C S_{a j}^{q^{T}}(x) C \gamma_{5}\right] \cdot \operatorname{Tr}\left[S_{m d}^{q}(-x) \gamma_{\mu} \gamma_{5} C S_{l e}^{c^{T}}(-x) C \gamma_{5}\right] & + \\
+ & \operatorname{Tr}\left[S_{b i}^{c}(x) \gamma_{5} C S_{a j}^{q^{T}}(x) C \gamma_{5} \gamma^{\mu}\right] \cdot \operatorname{Tr}\left[S_{m d}^{q}(-x) \gamma_{5} C S_{l e}^{c^{T}}(-x) C \gamma_{5} \gamma_{\mu}\right] & + \\
+ & \operatorname{Tr}\left[S_{b i}^{c}(x) \gamma^{\mu} \gamma_{5} C S_{a j}^{q^{T}}(x) C \gamma_{5} \gamma_{\mu}\right] \cdot \operatorname{Tr}\left[S_{m d}^{q}(-x) \gamma_{5} C S_{l e}^{c^{T}}(-x) C \gamma_{5}\right]
\end{array}\right\}
\end{aligned}
$$

onde $S^{c}(x)$ e $S^{q}(x)$ são os propagadores dos quarks pesados (charm) e leves $(q=u p$, down, strange), respectivamente. Note que, os antiquarks podem ser interpretados como quarks que se propagam "para trás" no espaço-tempo. Isso explica o uso do sinal negativo na coordenada $x$ dos propagadores. 
Nós trabalhamos com os propagadores dos quarks leves no espaço das coordenadas:

$$
S_{i j}^{q}(x)=\left\langle 0\left|T\left[q_{a}(x) \bar{q}_{b}(0)\right]\right| 0\right\rangle .
$$

Enquanto que, os propagadores dos quarks pesados são levados para o espaço dos momentos, via Transformada de Fourier:

$$
S_{i j}^{c}(x)=\int \frac{d^{4} p_{1}}{(2 \pi)^{4}} e^{-i p_{1} \cdot x} S_{i j}^{c}\left(p_{1}\right) .
$$

Com isto, a Eq. (3.12) é reescrita como:

$$
\begin{aligned}
& \Pi\left(q^{2}\right)=-\frac{i \epsilon_{a b c} \epsilon_{d e c} \epsilon_{i j k} \epsilon_{l m k}}{6 q^{2}} \int \frac{d^{4} x d^{4} p_{1} d^{4} p_{2}}{(2 \pi)^{8}} e^{i q \cdot x} e^{-i p_{1} \cdot x} e^{-i p_{2} \cdot x}
\end{aligned}
$$

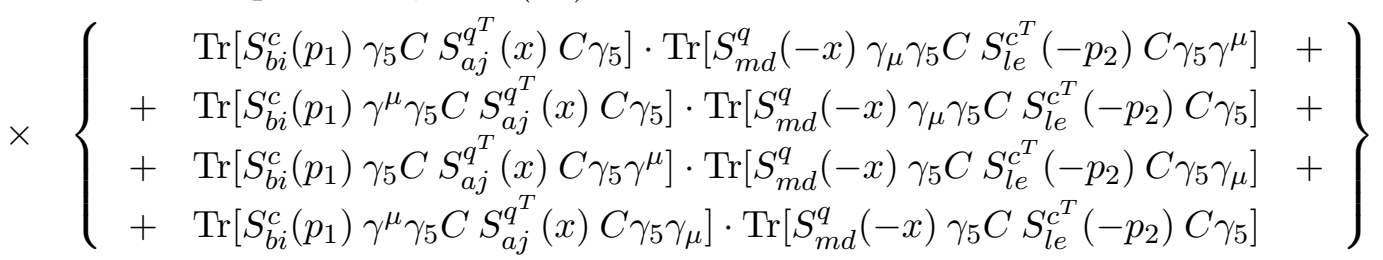

Por simplicidade, a representação dos diagramas de um tetraquark foi construído de tal forma que as duas linhas superiores representassem os propagadores dos quarks e as duas linhas inferiores representassem os propagadores dos antiquarks. Assim, por exemplo, o diagrama perturbativo de um tetraquark é representado por:

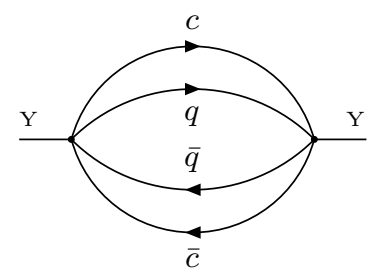

Para calcular todas as contribuições advindas da função de correlação, precisamos das expressões dos propagadores dos quarks. Uma lista dos propagadores utilizados neste trabalho pode ser encontrada em diversos trabalhos $[44,40]$ e um resumo é apresentado no Apêndice A.

Nesta seção, calculamos as integrais contidas em $\Pi\left(q^{2}\right)$, seguindo alguns procedimentos esquematizados abaixo:

- definimos os diagramas que contribuem para a OPE;

- inserimos os propagadores necessários conforme a construção de cada diagrama;

- utilizamos as relações das seções 5 e 6 do Apêndice B para: calcular os traços e resolver as integrais nas variáveis $x, p_{1}$ e $p_{2}$;

- escrevemos $\Pi\left(q^{2}\right)$ como uma relação de dispersão;

- a partir da relação (B.34), determinamos a densidade espectral no Lado da QCD.

Para esta QCDSR, consideramos as contribuições do diagrama perturbativo $(D=0)$ e a contribuição dos diagramas não-perturbativos e não-fatorizáveis (com dimensão até $D=8$ ). 
Para cada dimensão da OPE, calculamos a contribuição do termo dominante e, quando necessário, admitimos correções lineares com relação a massa do quark leve $s$. Por fim, para cada dimensão determinamos uma densidade espectral correspondente, de forma que a expressão para a densidade espectral total, $\rho^{\text {ope }}(s)$, fica dada por:

$$
\rho^{\text {ope }}(s)=\rho^{\text {pert }}(s)+\rho^{\langle\bar{q} q\rangle}(s)+\rho^{\left\langle G^{2}\right\rangle}(s)+\rho_{1}^{\text {mix }}(s)+\rho_{2}^{\text {mix }}(s)+\rho^{\langle\bar{q} q\rangle^{2}}(s)+\rho^{\text {mix }\langle\bar{q} q\rangle}(s)
$$

Apesar do condensado de dimensão 8 desempenhar, em geral, um papel secundário nos cálculos de regras de soma, vamos incluí-lo no nosso cálculo para verificar se este comportamento é verificado nos estudos da família de mésons $Y$.

Os detalhes do cálculo da função de correlação (3.15) são mostrados no Apêndice C.

(a) Perturbativos $D=0$

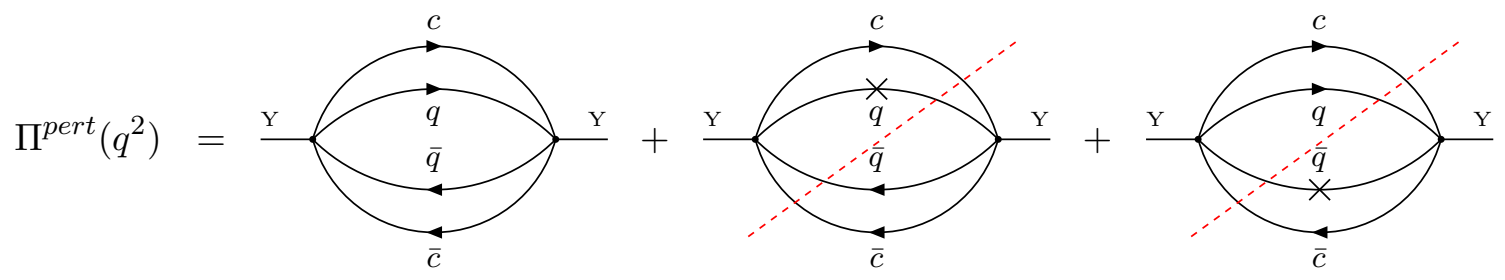

$$
\begin{aligned}
\Pi^{\text {pert }}\left(q^{2}\right)=\frac{1}{3 \cdot 2^{8} \pi^{6} q^{2}} \int_{0}^{1} \frac{d \alpha d \beta}{\alpha^{3} \beta^{3}}(1-\alpha-\beta)\left[m_{c}^{2}(\alpha+\beta)-\alpha \beta q^{2}\right]^{3} \times \\
\quad \times\left[m_{c}^{2}(1-2 \alpha-2 \beta)+\alpha \beta q^{2}\right] \ln \left[m_{c}^{2}(\alpha+\beta)-\alpha \beta q^{2}\right]
\end{aligned}
$$

Foram consideradas as correções nas massas dos quarks leves, representadas pelos diagramas que possuem o símbolo $\times$ sobre o propagador do quark $q$ e do seu respectivo antiquark. A contribuição desses diagramas, entretanto, se cancelam e isto é representado pela linha tracejada na figura acima.

A contribuição perturbativa para a densidade espectral é dada por $\left(s=q^{2}\right)$ :

$\rho^{p e r t}(s)=-\frac{1}{3 \cdot 2^{8} \pi^{6} s} \int_{\alpha_{\min }}^{\alpha_{\max }} \frac{d \alpha}{\alpha^{3}} \int_{\beta_{\min }}^{1-\alpha} \frac{d \beta}{\beta^{3}}(1-\alpha-\beta)\left[m_{c}^{2}(\alpha+\beta)-\alpha \beta s\right]^{3}\left[m_{c}^{2}(1-2 \alpha-2 \beta)+\alpha \beta s\right]$.

Os limites de integração que aparecem na densidade espectral $\rho^{\text {pert }}(s)$, e que serão os mesmos para as demais densidades espectrais (ver Apêndice C), são dados por:

$$
\begin{aligned}
\alpha_{\text {min }} & =\left(1-\sqrt{1-4 m_{c}^{2} / s}\right) / 2 \\
\alpha_{\max } & =\left(1+\sqrt{1-4 m_{c}^{2} / s}\right) / 2 \\
\beta_{\min } & =\alpha m_{c}^{2} /\left(s \alpha-m_{c}^{2}\right) .
\end{aligned}
$$


(b) Condensados de Quarks $D=3$
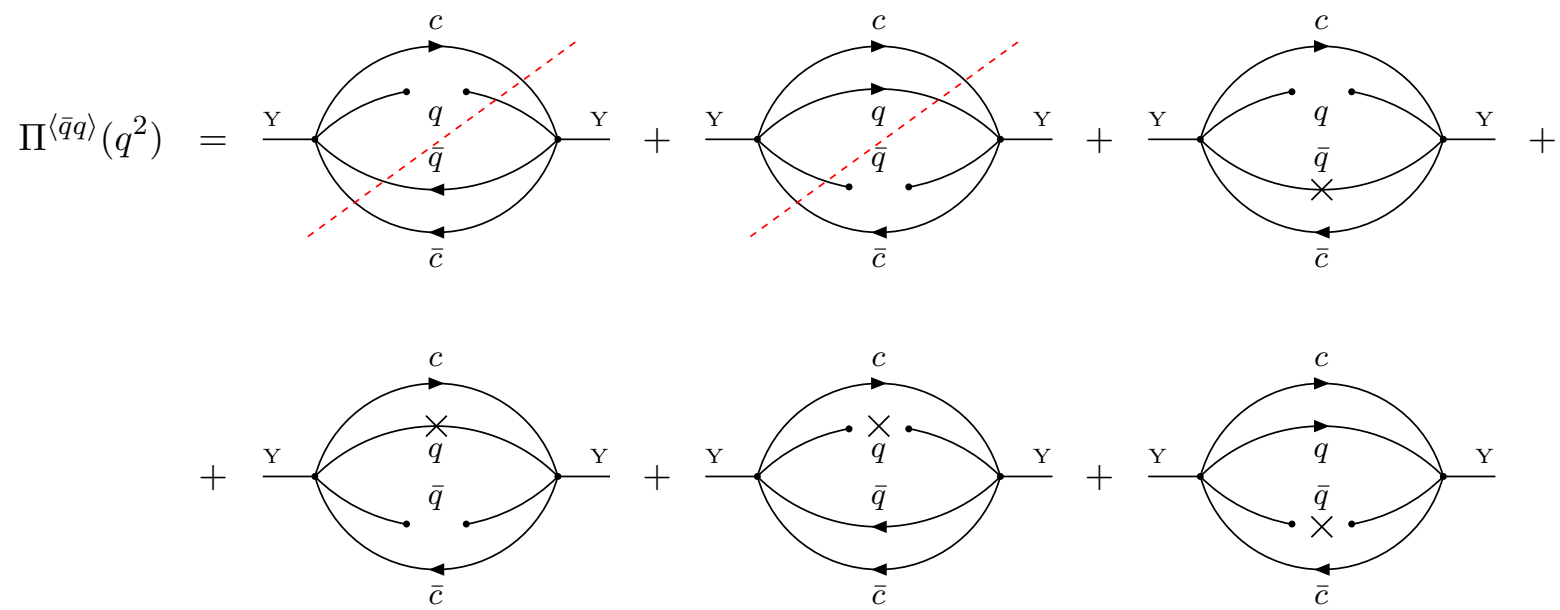

$\Pi^{\langle\bar{q} q\rangle}\left(q^{2}\right)=\frac{3 m_{q} m_{c}^{2}\langle\bar{q} q\rangle}{2^{4} \pi^{4} q^{2}} \int_{0}^{1} \frac{d \alpha d \beta}{\alpha \beta}\left[m_{c}^{2}(\alpha+\beta)-\alpha \beta q^{2}\right] \ln \left[m_{c}^{2}(\alpha+\beta)-\alpha \beta q^{2}\right]$

Aqui vemos que a contribuição do termo dominante é nula e, portanto, a contribuição dos condensados de quarks vem diretamente dos diagramas proporcionais à massa do quark leve $q$. Logo, no limite $m_{q} \rightarrow 0$, não há contribuição dos condensados de quarks. Este limite é razoável quando lidamos com os quarks leves $u\left(m_{u}=2.3 \mathrm{MeV}\right)$ e $d\left(m_{d}=6.4 \mathrm{MeV}\right)$ [16].

A contribuição dos condensados de quarks para a densidade espectral é dada por:

$$
\rho^{\langle\bar{q} q\rangle}(s)=-\frac{3 m_{q} m_{c}^{2}\langle\bar{q} q\rangle}{2^{4} \pi^{4} s} \int_{\alpha_{\min }}^{\alpha_{\max }} \frac{d \alpha}{\alpha} \int_{\beta_{\min }}^{1-\alpha} \frac{d \beta}{\beta}\left[m_{c}^{2}(\alpha+\beta)-\alpha \beta s\right] .
$$

(c) Condensados de Glúons $D=4$

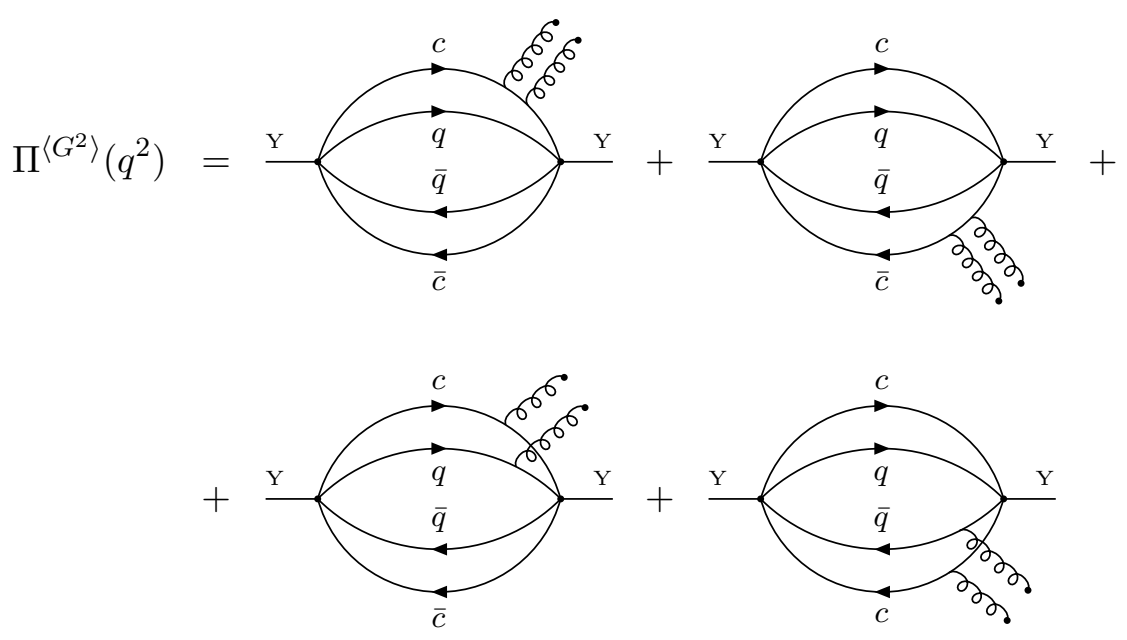

$\Pi^{\left\langle G^{2}\right\rangle}\left(q^{2}\right)=-\frac{m_{c}^{2}\left\langle g_{s}^{2} G^{2}\right\rangle}{3^{2} 2^{9} \pi^{6} q^{2}} \int_{0}^{1} \frac{d \alpha d \beta}{\beta^{3}}(1-\alpha-\beta)\left\{(7 \alpha-3)\left[m_{c}^{2}(\alpha+\beta)-\alpha \beta q^{2}\right]-m_{c}^{2} \alpha(1-\alpha-\beta)\right\} \ln \left[m_{c}^{2}(\alpha+\beta)-\alpha \beta q^{2}\right]$. 
A soma das contribuições dos diagramas não-perturbativos e não-fatorizáveis dos condensados de glúons para a densidade espectral nos fornece (ver Apêndice C):

$$
\rho^{\left\langle G^{2}\right\rangle}(s)=\frac{m_{c}^{2}\left\langle g_{s}^{2} G^{2}\right\rangle}{3^{2} 2^{9} \pi^{6} s} \int_{\alpha_{\min }}^{\alpha_{\max }} d \alpha \int_{\beta_{\min }}^{1-\alpha} \frac{d \beta}{\beta^{3}}(1-\alpha-\beta)\left\{(7 \alpha-3)\left[m_{c}^{2}(\alpha+\beta)-\alpha \beta s\right]-m_{c}^{2} \alpha(1-\alpha-\beta)\right\} .
$$

\section{(d.1) Condensado Misto $1 \quad D=5$}

Os condensados mistos podem ser formados de duas formas distintas: o glúon do condensado é emitido pelo quark leve $q$ (ou antiquark $\bar{q}$ ), ou pode ser emitido pelo quark pesado $c$ (ou antiquark $\bar{c})$.
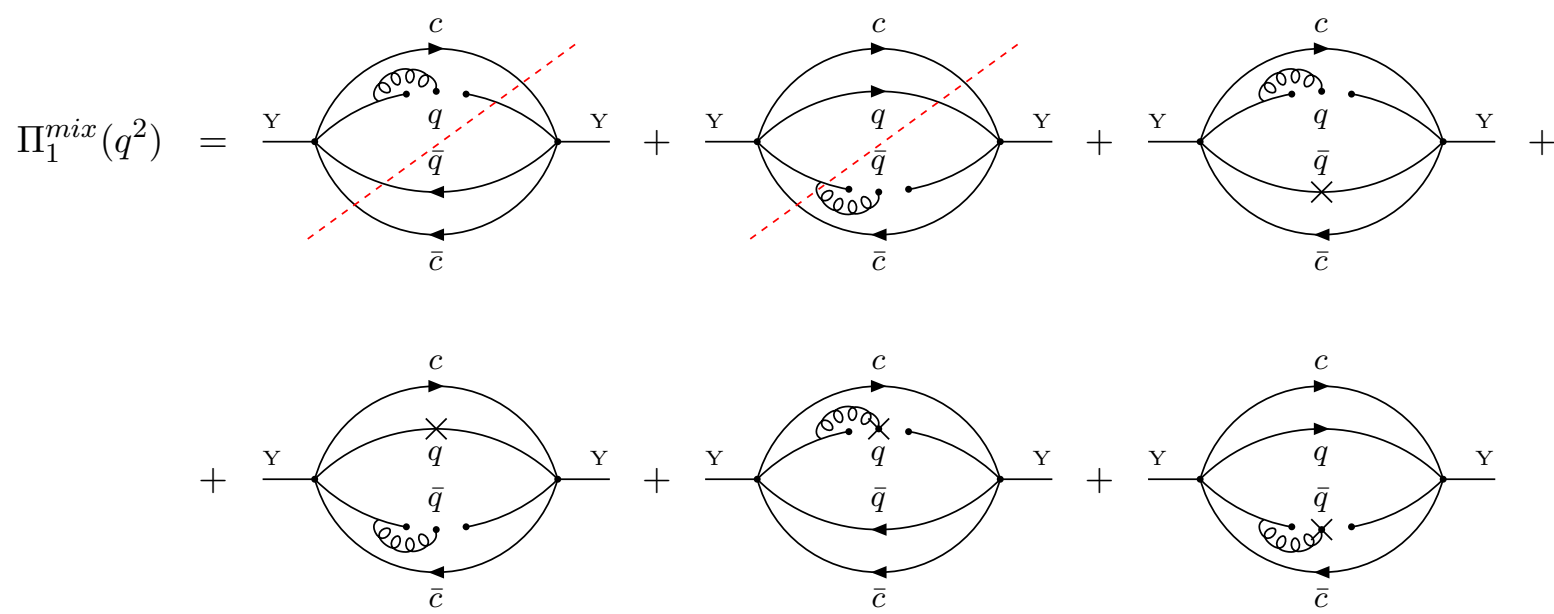

$\Pi_{1}^{m i x}\left(q^{2}\right)=\frac{m_{q}\left\langle\bar{q} g_{s} \sigma G q\right\rangle}{3 \cdot 2^{5} \pi^{4} q^{2}} \int_{0}^{1} d \alpha\left[5 m_{c}^{2}-\alpha(1-\alpha) q^{2}\right] \ln \left[m_{c}^{2}-\alpha(1-\alpha) q^{2}\right]$.

Novamente, observamos que os diagramas do termo principal se cancelam, fazendo com que apenas os termos proporcionais a $m_{q}$ contribuam para o condensado misto.

A contribuição do condensado misto 1 para a densidade espectral é dada por:

$$
\rho_{1}^{m i x}(s)=-\frac{m_{q}\left\langle\bar{q} g_{s} \sigma G q\right\rangle}{3 \cdot 2^{5} \pi^{4} s} \int_{\alpha_{\min }}^{\alpha_{\max }} d \alpha\left[5 m_{c}^{2}-\alpha(1-\alpha) s\right]
$$


(d.2) Condensado Misto $2 \quad D=5$
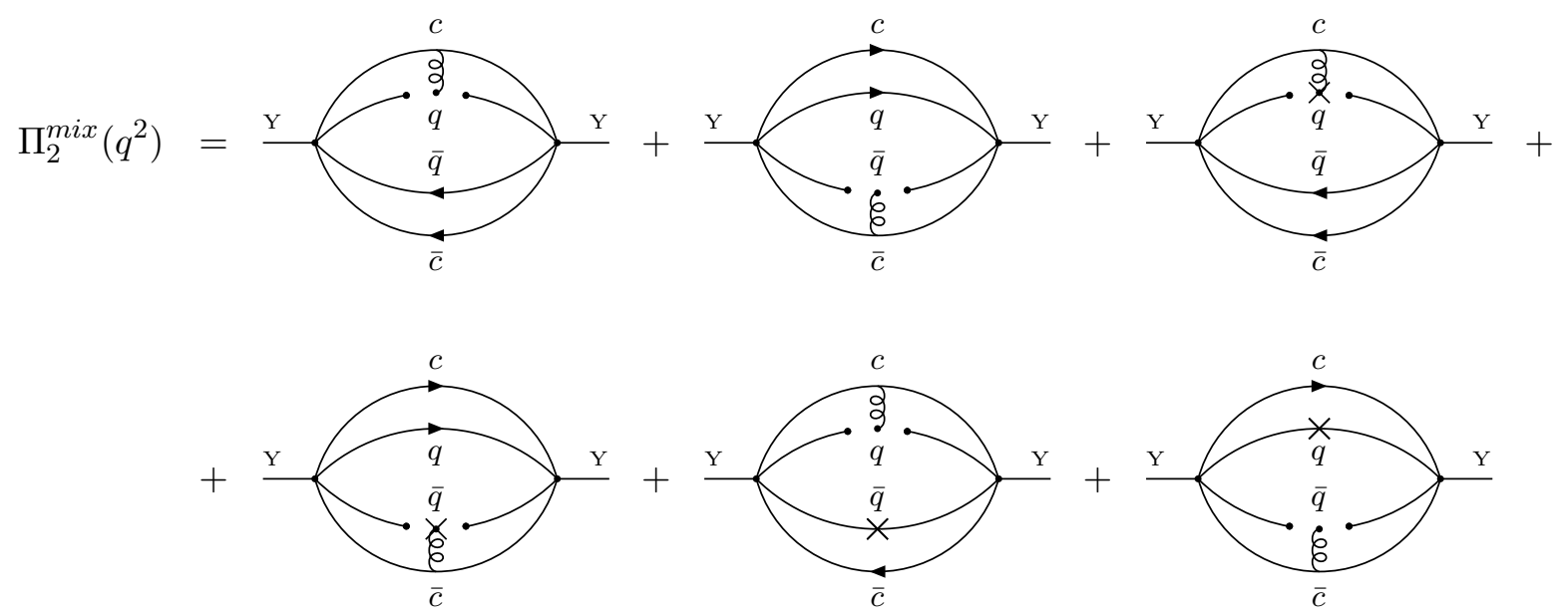

$$
\begin{aligned}
\Pi_{2}^{m i x}\left(q^{2}\right) & =-\frac{\left\langle\bar{q} g_{s} \sigma G q\right\rangle}{3 \cdot 2^{6} \pi^{4} q^{2}}\left\{\int_{0}^{1} \frac{d \alpha d \beta}{\alpha \beta^{2}}\left[m_{c}(2 \alpha+\beta)\left(m_{c}^{2}(\alpha+\beta)-\alpha \beta q^{2}\right)+5 m_{q} m_{c}^{2} \alpha \beta\right] \ln \left[m_{c}^{2}(\alpha+\beta)-\alpha \beta q^{2}\right]\right. \\
& \left.+m_{q} \int_{0}^{1} \frac{d \alpha}{(1-\alpha)}\left[m_{c}^{2}-\alpha(1-\alpha) q^{2}\right] \ln \left[m_{c}^{2}-\alpha(1-\alpha) q^{2}\right]\right\} .
\end{aligned}
$$

A contribuição do condensado misto não-fatorizável para a densidade espectral é dada por:

$$
\begin{array}{r}
\rho_{2}^{\operatorname{mix}}(s)=\frac{\left\langle\bar{q} g_{s} \sigma G q\right\rangle}{3 \cdot 2^{6} \pi^{4} s}\left\{\int_{\alpha_{\min }}^{\alpha_{\max }} \frac{d \alpha}{\alpha} \int_{\beta_{\min }}^{1-\alpha} \frac{d \beta}{\beta^{2}}\left[m_{c}(2 \alpha+\beta)\left(m_{c}^{2}(\alpha+\beta)-\alpha \beta s\right)+5 m_{q} m_{c}^{2} \alpha \beta\right]+\right. \\
\left.\quad+m_{q} \int_{\alpha_{\min }}^{\alpha_{\max }} \frac{d \alpha}{(1-\alpha)}\left[m_{c}^{2}-\alpha(1-\alpha) s\right]\right\}
\end{array}
$$

É interessante notar que no limite em que $m_{q} \rightarrow 0$, temos:

$$
\begin{gathered}
\rho_{1}^{\operatorname{mix}}(s) \rightarrow 0 \\
\rho_{2}^{\operatorname{mix}}(s) \rightarrow \frac{m_{c}\left\langle\bar{q} g_{s} \sigma G q\right\rangle}{3 \cdot 2^{6} \pi^{4} s} \int_{\alpha_{\min }}^{\alpha_{\max }} \frac{d \alpha}{\alpha} \int_{\beta_{\min }}^{1-\alpha} \frac{d \beta}{\beta^{2}}\left\{(2 \alpha+\beta)\left[m_{c}^{2}(\alpha+\beta)-\alpha \beta s\right]\right\} .
\end{gathered}
$$

Portanto, a contribuição dos condensados mistos neste limite é dada apenas pelos termos nãofatorizáveis presentes em $\rho_{2}^{\operatorname{mix}}(s)$. 
(e) Condensado de 4-Quarks $D=6$

$$
\begin{aligned}
& \langle\bar{q} q\rangle^{2}\left(q^{2}\right)= \\
& \Pi^{\langle\bar{q} q\rangle^{2}}\left(q^{2}\right)=\frac{\langle\bar{q} q\rangle^{2}}{3 \cdot 2^{2} \pi^{2} q^{2}} \int_{0}^{1} d \alpha\left[2 m_{c}^{2}+\alpha(1-\alpha) q^{2}\right] \ln \left[m_{c}^{2}-\alpha(1-\alpha) q^{2}\right] .
\end{aligned}
$$

A contribuição do condensado triplo de glúons, que possui $D=6$, foi desprezada por ser suprimida por um fator de loop $1 /\left(16 \pi^{2}\right)$.

A contribuição do condensado de 4-Quarks para a densidade espectral é dada por:

$$
\rho^{\langle\bar{q} q\rangle^{2}}(s)=-\frac{\langle\bar{q} q\rangle^{2}}{3^{2} \cdot 2^{2} \pi^{2}}\left(\frac{5 m_{c}^{2}}{s}-\frac{1}{2}\right) \sqrt{1-4 m_{c}^{2} / s} .
$$

(f) Condensado de Quarks Misto $D=8$
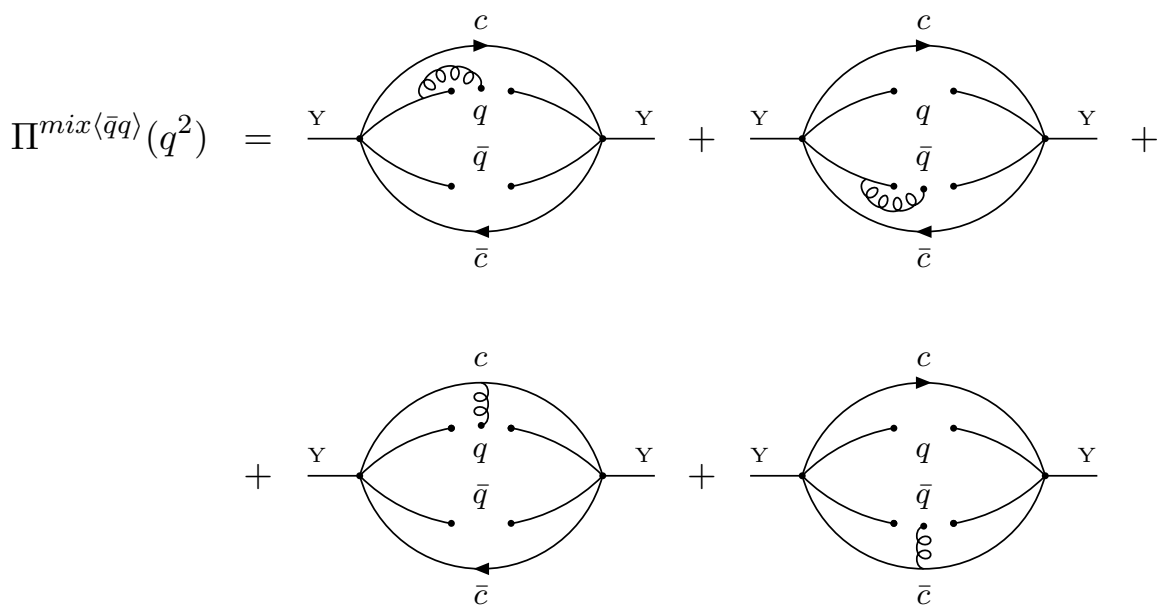

$$
\begin{array}{r}
\Pi^{m i x\langle\bar{q} q\rangle}\left(q^{2}\right)=\frac{\langle\bar{q} q\rangle\left\langle\bar{q} g_{s} \sigma G q\right\rangle}{3^{2} 2^{4} \pi^{2} q^{2}}\left[\frac{7}{6}-3 m_{c}^{2} \int_{0}^{1} d \alpha\left(\frac{\alpha}{m_{c}^{2}-\alpha(1-\alpha) q^{2}}-\frac{2 \alpha(1-\alpha) m_{c}^{2}}{\left[m_{c}^{2}-\alpha(1-\alpha) q^{2}\right]^{2}}\right)-\right. \\
\left.-2 \int_{0}^{1} d \alpha \alpha(6 \alpha-5) \ln \left[m_{c}^{2}-\alpha(1-\alpha) q^{2}\right]\right] .
\end{array}
$$

Devemos notar que há mais diagramas de dimensão 8 que poderiam ser considerados. Entretanto, a inclusão desses diagramas requer uma análise mais elaborada com relação às aproximações de fatorização que utilizamos até aqui [46], e que vão além dos objetivos deste projeto. 
Outro ponto importante é que os diagramas de dimensão 8 possuem uma parte imaginária proporcional a uma função delta de Dirac e que pode, portanto, ser facilmente integrada. Assim, além do termo proporcional a densidade espectral $\rho^{\operatorname{mix}\langle\bar{q} q\rangle}(s)$, temos outro termo que foi integrado diretamente da relação de dispersão e que é dado por (após a Transformada de Borel):

$$
\Pi^{m i x\langle\bar{q} q\rangle}\left(M^{2}\right)=-\frac{\langle\bar{q} q\rangle\left\langle\bar{q} g_{s} \sigma G q\right\rangle}{3^{2} 2^{4} \pi^{2}}\left(\frac{2}{3}-3 \int_{0}^{1} d \alpha\left[\alpha-2 \alpha^{2}+\frac{2 m_{c}^{2}}{M^{2}}\right] e^{-\frac{m_{c}^{2}}{\alpha(1-\alpha) M^{2}}}\right) .
$$

Já a contribuição dos condensados de quarks misto para a densidade espectral é dada por:

$$
\rho^{m i x\langle\bar{q} q\rangle}(s)=-\frac{\langle\bar{q} q\rangle\left\langle\bar{q} g_{s} \sigma G q\right\rangle}{3^{2} 2^{4} \pi^{2} s}\left(1+4 m_{c}^{2} / s\right) \sqrt{1-4 m_{c}^{2} / s} .
$$

Obtidas as expressões das densidades espectrais, podemos realizar a comparação entre os lados da teoria para extrair a massa do hádron descrito pela corrente (3.1).

\subsubsection{Dualidade Quark-Hadron}

Uma informação importante que surge como consequência direta do cálculo da densidade espectral é a seguinte imposição à variável $s$ :

$$
s_{\min }=4 m_{c}^{2} .
$$

Considerando que os condensados de dimensão 8 dão origem aos termos integrados diretamente da relação de dispersão, a equação (2.76) é ligeiramente modificada da seguinte forma:

$$
f_{Y}^{2} m_{Y}^{6} e^{-m^{2} / M^{2}}=\int_{4 m_{c}^{2}}^{s_{0}} d s \rho^{o p e}(s) e^{-s / M^{2}}+\Pi^{m i x\langle\bar{q} q\rangle}\left(M^{2}\right) .
$$

Derivando a expressão acima, com relação à $\left(1 / M^{2}\right)$, obtemos:

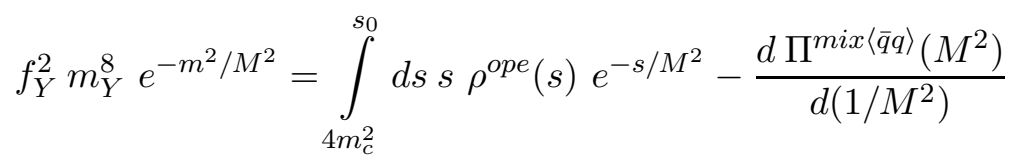

onde a derivada de $\Pi^{m i x\langle\bar{q} q}\left(M^{2}\right)$ é dada por:

$$
\frac{d \Pi^{\operatorname{mix}\langle\bar{q} q\rangle}\left(M^{2}\right)}{d\left(1 / M^{2}\right)}=\frac{m_{c}^{2}\langle\bar{q} q\rangle\left\langle\bar{q} g_{s} \sigma G q\right\rangle}{3 \cdot 2^{4} \pi^{2}} \int_{0}^{1} d \alpha\left[\frac{\alpha-2 m_{c}^{2} / M^{2}}{\alpha(1-\alpha)}\right] e^{-\frac{m_{c}^{2}}{\alpha(1-\alpha) M^{2}}} .
$$

Assim, a equação (2.78) para obtenção da massa do tetraquark fica:

$$
m_{Y}^{2}=\frac{\int_{4 m_{c}^{2}}^{s_{0}} d s s \rho^{o p e}(s) e^{-s / M^{2}}-\frac{d \Pi^{m i x\langle\bar{q} q\rangle}\left(M^{2}\right)}{d\left(1 / M^{2}\right)}}{\int_{4 m_{c}^{2}}^{s_{0}} d s \rho^{o p e}(s) e^{-s / M^{2}}+\Pi^{m i x\langle\bar{q} q\rangle}\left(M^{2}\right)} .
$$




\subsubsection{Resultados Numéricos}

Para determinar o limiar do contínuo, $\sqrt{s_{0}}$, procedemos da seguinte forma:

- fixamos um valor arbitrário para $\sqrt{s_{0}} \mathrm{e}$,

- em seguida, calculamos o valor da massa através da Eq. (3.29);

- se a diferença entre o valor de $\sqrt{s_{0}}$ e a massa experimental do hádron for em torno de $0.5 \mathrm{MeV}$, então o valor fixado inicialmente para $\sqrt{s_{0}}$ é aceito.

Para o cálculo numérico utilizamos os seguintes valores [41, 44, 47]:

$$
\begin{array}{rlrl}
m_{c}\left(m_{c}\right) & =(1.23 \pm 0.05) \mathrm{MeV} & m_{u} & =2.3 \mathrm{MeV} \\
m_{d} & =6.4 \mathrm{MeV} & m_{q} & \equiv\left(m_{u}+m_{d}\right) / 2=4.3 \mathrm{MeV} \\
m_{s} & =(0.13 \pm 0.03) \mathrm{GeV} & \langle\bar{q} q\rangle & =-(0.23 \pm 0.03)^{3} \mathrm{GeV}^{3} \\
\left\langle g_{s}^{2} G^{2}\right\rangle & =0.88 \mathrm{GeV}^{4} & \left\langle\bar{q} g_{s} \sigma G q\right\rangle & =m_{0}^{2}\langle\bar{q} q\rangle \\
m_{0}^{2} & =0.8 \mathrm{GeV}^{2} & \langle\bar{s} s\rangle & =0.8\langle\bar{q} q\rangle \\
\left\langle\bar{s} g_{s} \sigma G s\right\rangle & =m_{0}^{2}\langle\bar{s} s\rangle &
\end{array}
$$

Analisaremos os dois possíveis tetraquarks que podem ser descritos pela corrente (3.1).

\section{(a) Tetraquark com Estranheza}

Considerando o quark estranho na corrente da Eq. (3.1), seu conteúdo de quarks fica dado por: $c s \bar{c} \bar{s}$.

O limite superior para $M^{2}$ é obtido identificando a região onde a contribuição do pólo é maior do que a do contínuo. Para isto, a Figura (3.1) mostra as contribuições relativas do contínuo (linha cheia) e do pólo (linha pontilhada), para $\sqrt{s_{0}}=5.1 \mathrm{GeV}$. Neste caso, temos $M_{\max }^{2}=4.1 \mathrm{GeV}^{2}$.

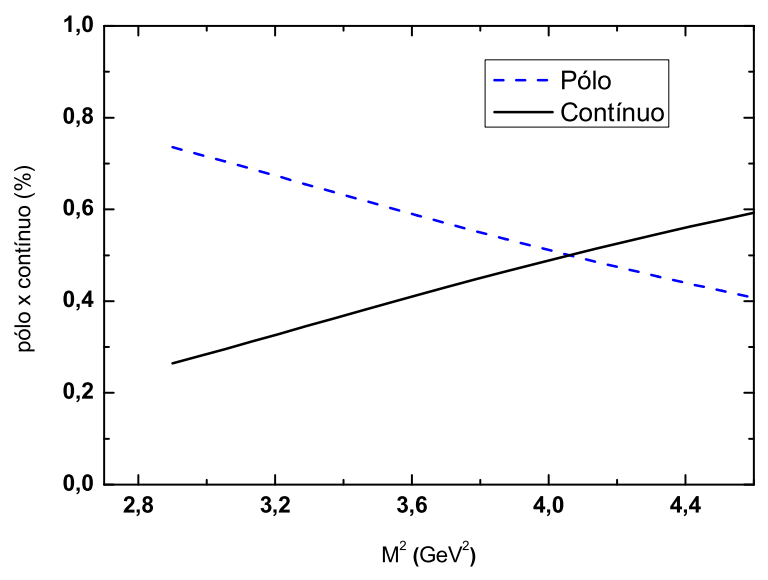

Figura 3.1: Relação Pólo x Contínuo, para $\sqrt{s_{0}}=5.1 \mathrm{GeV}$.

Foram realizados cálculos com três valores distintos para $\sqrt{s_{0}}$. Os respectivos limites superiores de $M^{2}$ são listados na tabela abaixo: 
Tabela I: Limites superiores da Janela de Borel para as QCDSR.

\begin{tabular}{|c|c|}
\hline$\sqrt{s_{0}}(\mathrm{GeV})$ & $M_{\max }^{2}\left(\mathrm{GeV}^{2}\right)$ \\
\hline 5.0 & 3.8 \\
\hline 5.1 & 4.1 \\
\hline 5.2 & 4.2 \\
\hline
\end{tabular}

Para determinarmos o limite inferior de $M^{2}$ devemos observar a convergência da OPE. Na Figura (3.2), obtemos uma boa convergência para $M_{m i n}^{2}=3.2 \mathrm{GeV}^{2}$. Ainda nesta figura, podemos observar que a contribuição do condensado de dimensão 8 é muito pequena. Devido ao fato de não estarmos considerando toda a classe possível de condensados de dimensão 8, sua contribuição será desconsiderada no decorrer deste trabalho.

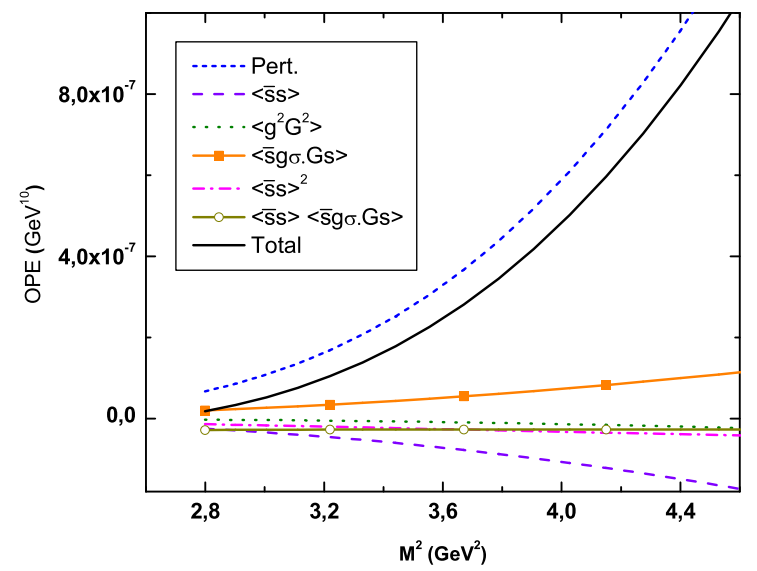

Figura 3.2: A convergência da OPE utilizando $\sqrt{s_{0}}=5.1 \mathrm{GeV}$.

Assim, a janela de Borel desta QCDSR é dada por: $3.2 \leq M^{2} \leq 4.2 \mathrm{GeV}^{2}$, para o intervalo de $s_{0}: 5.0 \leq \sqrt{s_{0}} \leq 5.2 \mathrm{GeV}$.

Na Figura (3.3), é mostrada a massa do tetraquark para diferentes valores de $s_{0}$. Observamos que há uma estabilidade razoável de $m_{Y}$ dentro da janela de Borel considerada.

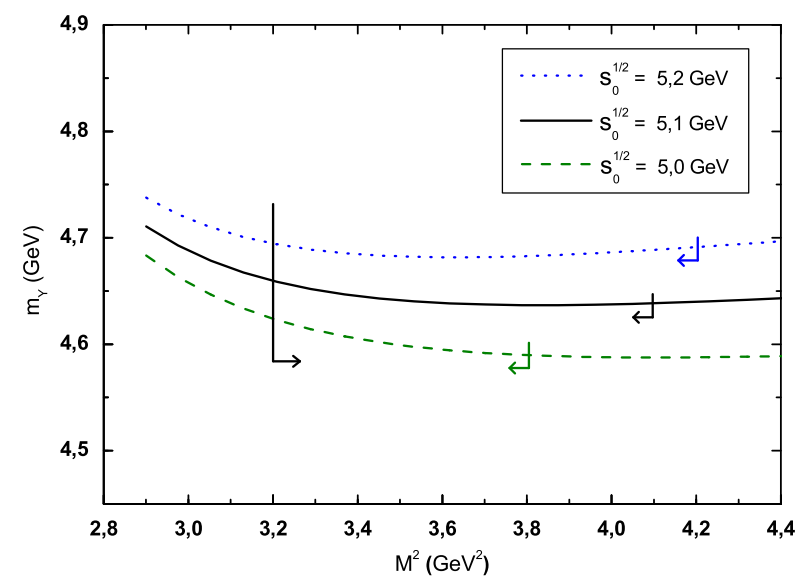

Figura 3.3: A massa do tetraquark com estranheza em função do parâmetro $M^{2}$, para diferentes valores de $\sqrt{s_{0}}$. As setas delimitam os limites da janela de Borel das QCDSR. O limite inferior é dado pela condição de convergência da OPE e o limite superior é dado pela dominância da contribuição do pólo.

Para checar a dependência dos nossos resultados com o valor da massa dos quarks e dos condensados, nós fixamos $\sqrt{s_{0}}=5.1 \mathrm{GeV}$ e variamos os seus valores de acordo com a suas 
respectivas incertezas. Dessa forma, foi possível verificar que a principal fonte de incerteza no valor obtido para a massa do tetraquark advém do limiar do contínuo. Adicionando os erros em quadratura, a massa obtida para a partícula correspondente ao tetraquark com estranheza é:

$$
m_{Y}=(4.65 \pm 0.10) \mathrm{GeV}
$$

que está em excelente acordo com a massa do méson $Y$ (4660). Portanto, concluímos que o méson $Y(4660)$ pode ser descrito por um tetraquark com estranheza, com uma configuração de spin dada por um diquark escalar e um diquark vetorial. Esse conteúdo de quarks é consistente com espectro de massa invariante do dipíon mostrado na ref. [32], que mostra que há uma indicação de que o canal de decaimento do méson $Y(4660)$ contenha um estado intermediário bem definido para o dipíon consistente com $f_{0}(980)$. É também muito interessante notar que o méson $f_{0}(980)$ é considerado como um estado de tetraquark [48]. Esse aspecto pode desempenhar um papel importante no decaimento do $Y$ (4660) desde que, como mostrado na ref. [49], é muito difícil explicar uma largura tão pequena para um estado inicial de quatro quarks decaindo em dois pares de quarks.

\section{(b) Tetraquark com Quarks Leves}

Considerando os quarks leves $q=u, d$ na Eq. (3.1), o conteúdo de quarks do tetraquark fica: $c q \bar{c} \bar{q}$. A corrente então fica dada por:

$$
j_{\mu}^{(q)}=\frac{\epsilon_{a b c} \epsilon_{d e c}}{\sqrt{2}}\left[\left(q_{a}^{T} C \gamma_{5} c_{b}\right)\left(\bar{q}_{d} \gamma_{\mu} \gamma_{5} C \bar{c}_{e}^{T}\right)+\left(q_{a}^{T} C \gamma_{5} \gamma_{\mu} c_{b}\right)\left(\bar{q}_{d} \gamma_{5} C \bar{c}_{e}^{T}\right)\right]
$$

O estado de isospin zero é descrito pela corrente:

$$
j_{\mu}^{I=0}=\frac{j_{\mu}^{(u)}+j_{\mu}^{(d)}}{\sqrt{2}}
$$

No entanto, no nível de aproximação que estamos trabalhando, correntes com diferentes isospin levam ao mesmo resultado. Nesta QCDSR, utilizamos o valor $m_{q}=7.0 \mathrm{MeV}$, que é o valor da média aritmética das massas dos quarks $u$ e $d$.

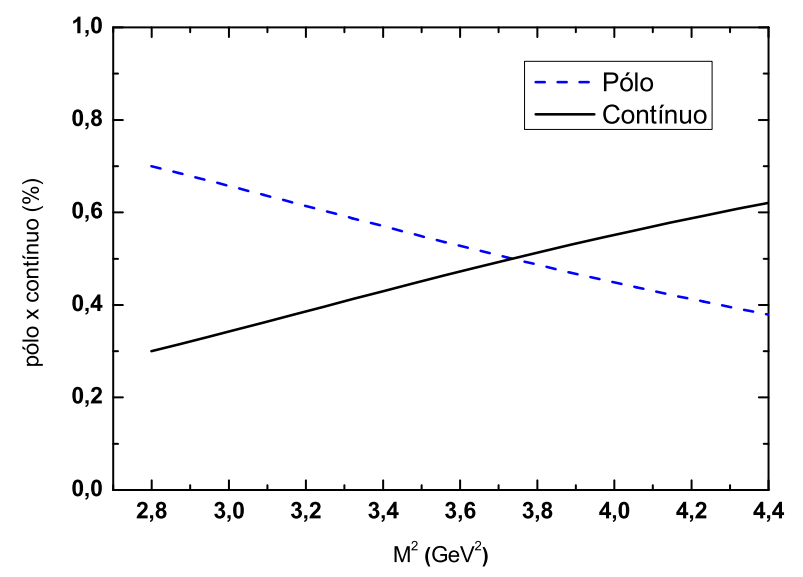

Figura 3.4: Relação Pólo x Contínuo, para $\sqrt{s_{0}}=4.9 \mathrm{GeV}$. 
Novamente, é necessário definirmos a janela de Borel para esta QCDSR. O limite superior para $M^{2}$ é obtido a partir da Figura (3.4), que mostra as contribuições relativas do contínuo (linha cheia) e do pólo (linha pontilhada), para $\sqrt{s_{0}}=4.9 \mathrm{GeV}$. Neste caso, temos $M_{\max }^{2}=$ $3.8 \mathrm{GeV}^{2}$.

Realizamos os cálculos com três valores distintos para $\sqrt{s_{0}}$. Os respectivos limites superiores de $M^{2}$ são listados na tabela abaixo:

Tabela II: Limites superiores da Janela de Borel para as QCDSR.

\begin{tabular}{|c|c|}
\hline$\sqrt{s_{0}}(\mathrm{GeV})$ & $M_{\max }^{2}\left(\mathrm{GeV}^{2}\right)$ \\
\hline 4.8 & 3.5 \\
\hline 4.9 & 3.8 \\
\hline 5.0 & 4.0 \\
\hline
\end{tabular}

Para determinarmos o limite inferior de $M^{2}$ devemos observar a convergência da OPE. Na Figura (3.5), obtemos uma boa convergência para $M_{\text {min }}^{2} \geq 3.2 \mathrm{GeV}^{2}$.

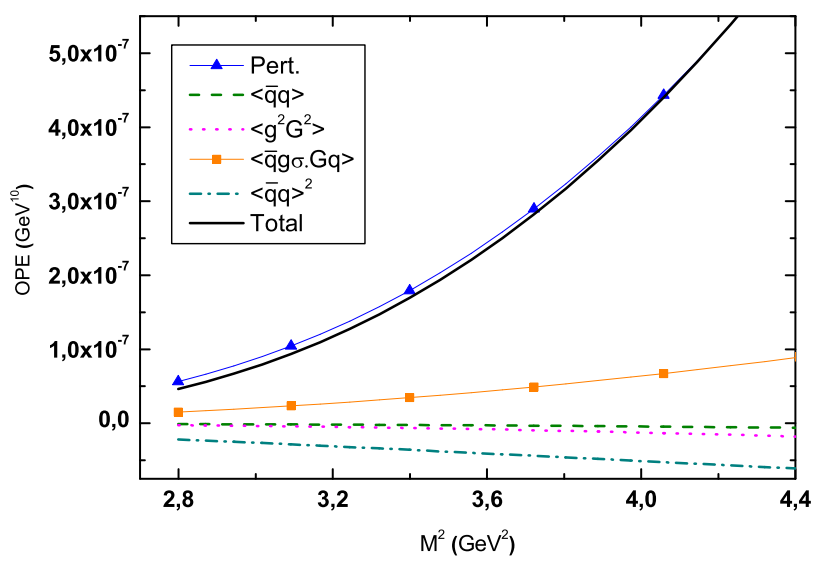

Figura 3.5: A convergência da OPE utilizando $\sqrt{s_{0}}=4.9 \mathrm{GeV}$.

Portanto, a janela de Borel desta QCDSR é dada por: $3.5 \leq M^{2} \leq 4.0 \mathrm{GeV}^{2}$, no intervalo de $s_{0}: 4.8 \leq \sqrt{s_{0}} \leq 5.0 \mathrm{GeV}$.

Na Figura (3.6), observamos uma certa instabilidade de $m_{Y}$ dentro da janela de Borel (indicada pelas setas). No entanto, ainda é possível estimar o valor obtido para a massa desse estado.

Levando em conta as variações em $M^{2}, s_{0},\langle\bar{q} q\rangle$ e $m_{c}$ dentro da janela de Borel, a massa da partícula correspondente ao tetraquark com quarks leves é:

$$
m_{Y}=(4.49 \pm 0.11) \mathrm{GeV}
$$

que é maior do que a massa do méson $Y(4360)$, mas é consistente com ela se considerarmos as incertezas.

Podemos novamente tentar extrair mais informações acerca do méson $Y(4360)$, analisando o espectro de massa invariante do dipíon [32]. Vemos que para o canal de decaimento do méson $Y(4360)$ o estado intermediário para o dipíon deve ter uma massa em torno de $600 \mathrm{MeV}$, que é consistente com o méson escalar $\sigma$ [36]. Neste caso, a estrutura interna do méson $Y(4360)$ é 


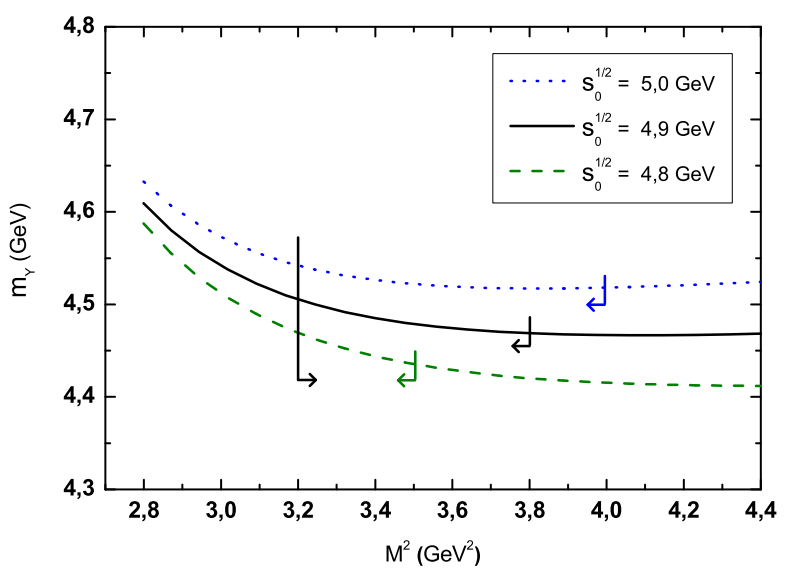

Figura 3.6: A massa do tetraquark com quark leve em função do parâmetro $M^{2}$, para diferentes valores de $\sqrt{s_{0}}$. As setas delimitam a janela de Borel das QCDSR.

mais compatível com uma corrente que não contenha quarks estranhos. Isto favorece o uso da corrente (3.32) para descrever o méson $Y(4360)$.

\subsection{O Estado Molecular}

Como discutido na referência [50], se o $X(3872)$ e $Z^{+}(4430)$ são de fato estados moleculares, então muitas outras moléculas podem existir. Em particular, as moléculas $D_{s 0}(2317) \bar{D}_{s}^{*}(2110)$ e $D_{0}(2352) \bar{D}^{*}(2010)$, com $J^{P C}=1^{--}$, poderiam também descrever os mésons Y. Logo, nesta seção consideramos as seguintes correntes méson-méson:

$$
\begin{gathered}
\tilde{j}_{s \mu}^{ \pm}=\frac{1}{\sqrt{2}}\left(D_{s \mu}^{*} \bar{D}_{s 0} \pm \bar{D}_{s \mu}^{*} D_{s 0}\right) \\
\tilde{j}_{q \mu}^{ \pm}=\frac{1}{\sqrt{2}}\left(D_{\mu}^{*} \bar{D}_{0} \pm \bar{D}_{\mu}^{*} D_{0}\right)
\end{gathered}
$$

onde o índice $s$ é empregado na corrente da molécula com estranheza e o sinal $( \pm)$ deve ser determinado pela conjugação de carga. Em termos de quarks, estas duas correntes podem ser condensadas em uma única expressão:

$$
\tilde{j}_{\mu}^{ \pm}=\frac{1}{\sqrt{2}}\left[\left(\bar{q} \gamma_{\mu} c\right)(\bar{c} q) \pm\left(\bar{c} \gamma_{\mu} q\right)(\bar{q} c)\right]
$$

quando $q=s$ obtemos a corrente (3.35) e quando $q=u, d$ obtemos a corrente (3.36).

Vamos então, à determinação dos sinais corretos destas correntes.

\subsubsection{Tranformações de Paridade e Conjugação de Carga}

Para determinar qual sinal devemos utilizar nas correntes, é necessário verificar qual escolha irá reproduzir os números quânticos $J^{P C}=1^{--}$. Fazendo a transformação de conjugação de 
carga da corrente (3.37), temos:

$$
\begin{aligned}
\mathcal{C} \tilde{j}_{\mu}^{ \pm} \mathcal{C}^{-1} & =\frac{1}{\sqrt{2}}\left[\left(-s^{T} C^{-1} \gamma_{\mu} C \bar{c}^{T}\right)\left(-c^{T} C^{-1} C \bar{s}^{T}\right) \pm\left(-c^{T} C^{-1} \gamma_{\mu} C \bar{s}^{T}\right)\left(-s^{T} C^{-1} C \bar{c}^{T}\right)\right] \\
& =\frac{1}{\sqrt{2}}\left[\left(s^{T} \gamma_{\mu}^{T} \bar{c}^{T}\right)\left(-c^{T} \bar{s}^{T}\right) \pm\left(c^{T} \gamma_{\mu}^{T} \bar{s}^{T}\right)\left(-s^{T} \bar{c}^{T}\right)\right] \\
& =\frac{1}{\sqrt{2}}\left[-\left(\bar{c} \gamma_{\mu} s\right)^{T}(\bar{s} c)^{T} \mp\left(\bar{s} \gamma_{\mu} c\right)^{T}(\bar{c} s)^{T}\right]
\end{aligned}
$$

como os diquarks são números, temos

$$
\mathcal{C} \tilde{j}_{\mu}^{ \pm} \mathcal{C}^{-1}=-\frac{1}{\sqrt{2}}\left[\left(\bar{c} \gamma_{\mu} s\right)(\bar{s} c) \pm\left(\bar{s} \gamma_{\mu} c\right)(\bar{c} s)\right]
$$

de tal forma que:

$$
\begin{aligned}
\mathcal{C} \tilde{j}_{\mu}^{+} \mathcal{C}^{-1} & =-\tilde{j}_{\mu}^{+} \\
\mathcal{C} \tilde{j}_{\mu}^{-} \mathcal{C}^{-1} & =\tilde{j}_{\mu}^{-}
\end{aligned}
$$

ou seja, a combinação (+) tem conjugação de carga negativa e a combinação $(-)$ tem conjugação de carga positiva. Logo, a corrente que apresenta o número quântico de conjugação de carga correto é:

$$
\tilde{j}_{\mu} \equiv \tilde{j}_{\mu}^{+}=\frac{1}{\sqrt{2}}\left[\left(\bar{s} \gamma_{\mu} c\right)(\bar{c} s)+\left(\bar{c} \gamma_{\mu} s\right)(\bar{s} c)\right]
$$

Quanto à paridade desta corrente, podemos avaliá-la da seguinte forma: os mésons escalares têm paridade positiva e os mésons vetoriais têm paridade negativa, por exemplo,

$$
\begin{aligned}
\mathcal{P} \bar{D}_{s 0} \mathcal{P}^{-1} & =\bar{D}_{s 0} \\
\mathcal{P} D_{s \mu}^{*} \mathcal{P}^{-1} & =-D_{s \mu}^{*}
\end{aligned}
$$

logo, (3.40) se transforma por paridade como:

$$
\mathcal{P} \tilde{j}_{\mu} \mathcal{P}^{-1}=-\tilde{j}_{\mu}
$$

e, portanto, reproduz todos os números quânticos para descrever a família de mésons $Y$.

\subsubsection{Lado Fenomenológico}

No lado fenomenológico não importa se os estados são compostos por tetraquarks ou moléculas. Logo, o tratamento da função de correlação dos estados moleculares é o mesmo que o da seção 3.1. Assim, o acoplamento do méson vetorial $Y$ à corrente (3.40) é dado pela mesma parametrização:

$$
\left\langle 0\left|j_{\mu}\right| Y\right\rangle=f_{Y} m_{Y}^{4} \epsilon_{\mu} \equiv \lambda \epsilon_{\mu}
$$

Portanto, para os estados moleculares, a função de correlação também será equivalente à 
(3.8):

$$
\Pi^{f e n}\left(q^{2}\right)=f_{Y}^{2} m_{Y}^{6} e^{-m^{2} / M^{2}}+\int_{s_{0}}^{\infty} d s \rho^{o p e}(s) e^{-m^{2} / M^{2}}
$$

\subsubsection{Lado da QCD}

Inserindo a corrente (3.40) na função de correlação (2.1), obtemos:

$$
\begin{aligned}
& \tilde{\Pi}\left(q^{2}\right)=-\frac{i}{6 q^{2}} \int \frac{d^{4} x d^{4} p_{1} d^{4} p_{2}}{(2 \pi)^{8}} e^{i q \cdot x} e^{-i p_{1} \cdot x} e^{-i p_{2} \cdot x} \\
& \times\left\{\begin{array}{rcc}
\operatorname{Tr}\left[\gamma_{\mu} S_{a i}^{c}\left(p_{1}\right) S_{i a}^{q}(-x)\right] \cdot \operatorname{Tr}\left[S_{b j}^{q}(x) \gamma^{\mu} S_{j b}^{c}\left(-p_{2}\right)\right] & + \\
+\operatorname{Tr}\left[S_{a i}^{c}\left(p_{1}\right) \gamma^{\mu} S_{i a}^{q}(-x)\right] \cdot \operatorname{Tr}\left[\gamma_{\mu} S_{b j}^{q}(x) S_{j b}^{c}\left(-p_{2}\right)\right] & + \\
+\operatorname{Tr}\left[\gamma_{\mu} S_{a i}^{c}\left(p_{1}\right) \gamma^{\mu} S_{i a}^{q}(-x)\right] \cdot \operatorname{Tr}\left[S_{b j}^{q}(x) S_{j b}^{c}\left(-p_{2}\right)\right] & + \\
+\operatorname{Tr}\left[S_{a i}^{c}\left(p_{1}\right) S_{i a}^{q}(-x)\right] \cdot \operatorname{Tr}\left[\gamma_{\mu} S_{b j}^{q}(x) \gamma^{\mu} S_{j b}^{c}\left(-p_{2}\right)\right] . &
\end{array}\right\}
\end{aligned}
$$

Na representação dos diagramas para os estados moleculares colocamos as duas linhas superiores (linha cheia) representando o primeiro méson e duas linhas inferiores (linha pontilhada) representando o segundo méson. Por exemplo, o diagrama perturbativo de uma molécula dado por (3.40) é:

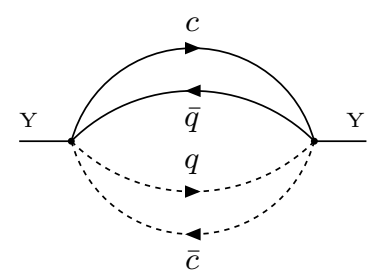

Utilizando novamente as expressões dos propagadores dos quarks e antiquarks, contidas no Apêndice A, podemos calcular as contribuições para a função de correlação.

No Lado da QCD, serão consideradas as contribuições dos diagramas perturbativos de primeira ordem em $m_{s}$ e as contribuições dos diagramas não-perturbativos e não-fatorizáveis com dimensão até $D=6$. A densidade espectral total, $\tilde{\rho}(s)$, é dada por:

$$
\tilde{\rho}(s)=\tilde{\rho}^{\text {pert }}(s)+\tilde{\rho}^{\langle\bar{q} q\rangle}(s)+\tilde{\rho}^{\left\langle G^{2}\right\rangle}(s)+\tilde{\rho}_{1}^{\text {mix }}(s)+\tilde{\rho}_{2}^{\text {mix }}(s)+\tilde{\rho}^{\langle\bar{q} q\rangle^{2}}(s) .
$$

O resultado obtido para a função de correlação dos estados moleculares, contido no Apêndice $\mathrm{D}$, demonstra as seguintes relações entre as densidades espectrais dos estados de tetraquarks, $\rho(s)$, e as dos estados moleculares, $\tilde{\rho}(s)$ :

- para as contribuições perturbativas ( $\left.\tilde{\rho}^{\text {pert }}\right)$ e não-perturbativas $\left(\tilde{\rho}^{(\bar{q} q\rangle}, \tilde{\rho}^{\left\langle G^{2}\right\rangle}, \tilde{\rho}_{1}^{m i x}, \tilde{\rho}^{\langle\bar{q} q\rangle^{2}}\right)$ :

$$
\tilde{\rho}(s)=\frac{3}{4} \rho(s)
$$

- para as contribuições não-fatorizáveis $\left(\rho^{\left\langle G^{2}\right\rangle}, \rho_{2}^{\text {mix }}\right)$ :

$$
\tilde{\rho}(s)=\frac{3}{2} \rho(s)
$$

A seguir, são listados os diagramas e as densidades espectrais para cada dimensão conside- 
rada nesta QCDSR.

(a) Perturbativos $D=0$

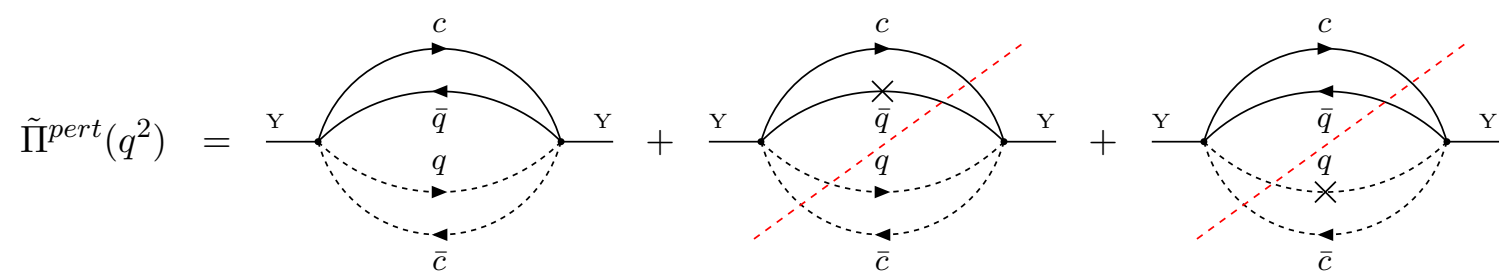

A contribuição perturbativa para a densidade espectral é dada por:

$$
\begin{aligned}
& \tilde{\rho}^{\text {pert }}(s)=\frac{3}{4} \rho^{\text {pert }}(s) \\
& \tilde{\rho}^{\text {pert }}(s)=-\frac{1}{2^{10} \pi^{6} s} \int_{\alpha_{\min }}^{\alpha_{\max }} \frac{d \alpha}{\alpha^{3}} \int_{\beta_{\text {min }}}^{1-\alpha} \frac{d \beta}{\beta^{3}}(1-\alpha-\beta)\left[m_{c}^{2}(\alpha+\beta)-\alpha \beta s\right]^{3}\left[m_{c}^{2}(1-2 \alpha-2 \beta)+\alpha \beta s\right]
\end{aligned}
$$

(b) Condensados de Quarks $D=3$
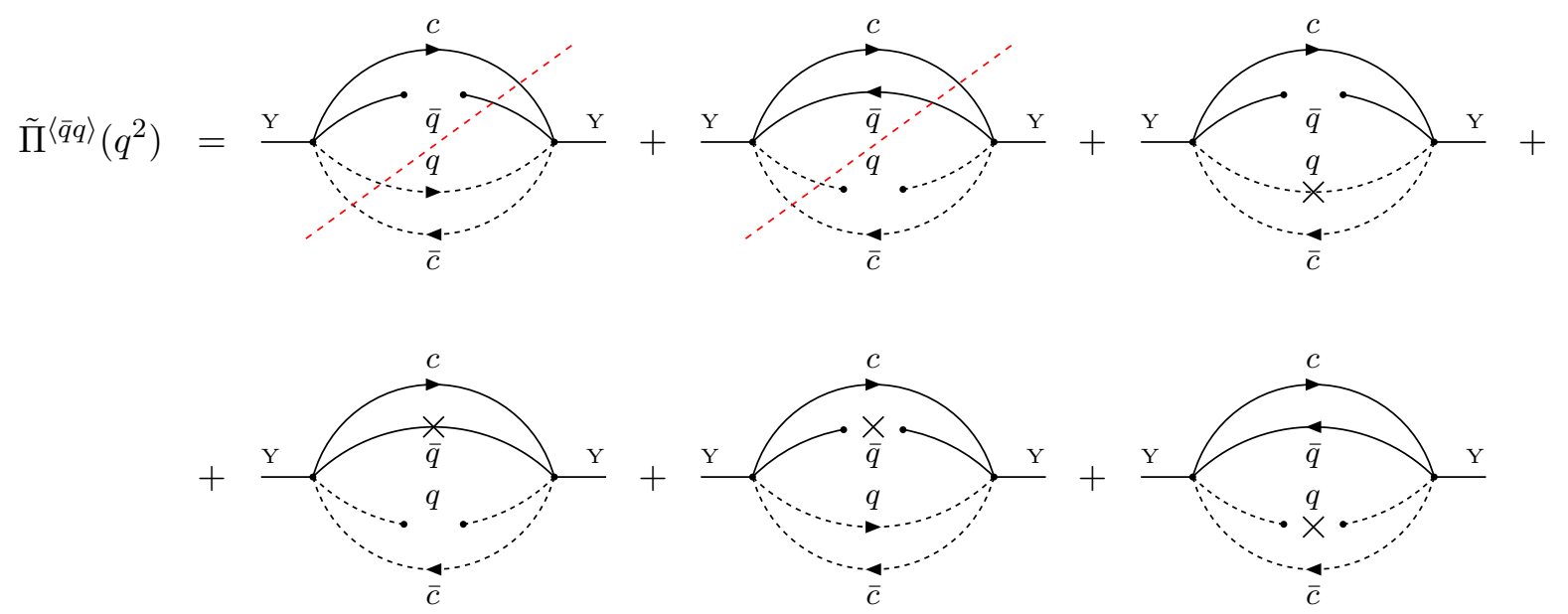

A contribuição dos condensados de quarks para a densidade espectral é dada por:

$$
\begin{aligned}
& \tilde{\rho}^{\langle\bar{q} q\rangle}(s)=\frac{3}{4} \rho^{\langle\bar{q} q\rangle}(s) \\
& \tilde{\rho}^{\langle\bar{q} q\rangle}(s)=-\frac{9 m_{q} m_{c}^{2}\langle\bar{q} q\rangle}{2^{6} \pi^{4} s} \int_{\alpha_{\min }}^{\alpha_{\max }} \frac{d \alpha}{\alpha} \int_{\beta_{\min }}^{1-\alpha} \frac{d \beta}{\beta}\left[m_{c}^{2}(\alpha+\beta)-\alpha \beta s\right] .
\end{aligned}
$$


(c) Condensados de Glúons $D=4$

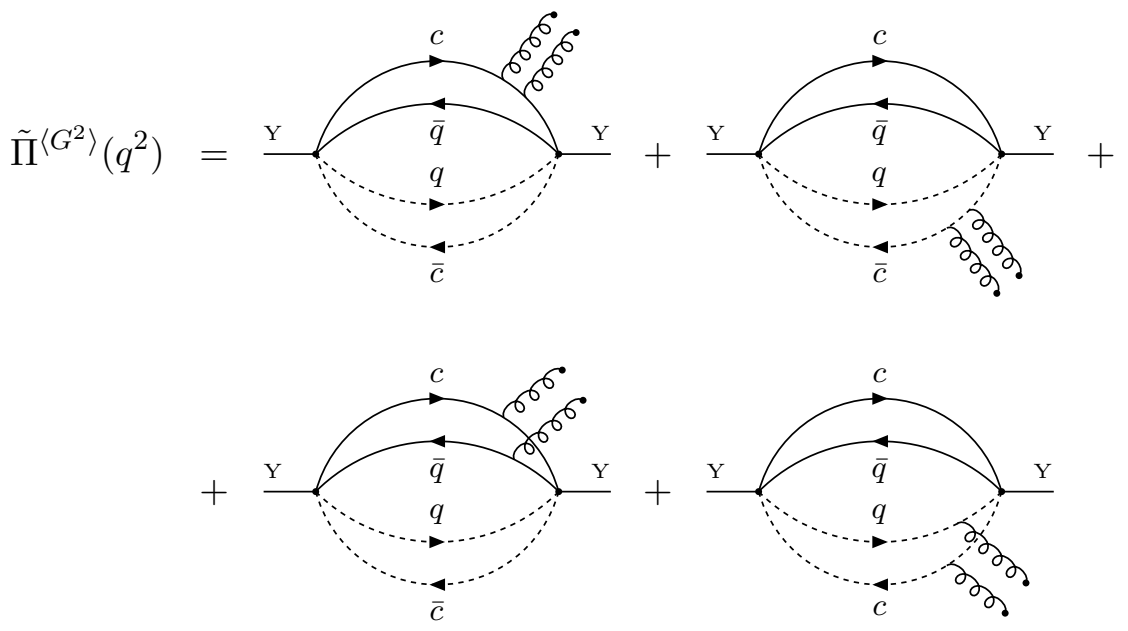

A soma das contribuições dos diagramas não-perturbativos e não-fatorizáveis dos condensados de glúons para a densidade espectral nos fornece (ver Apêndice D):

$$
\tilde{\rho}^{\left\langle G^{2}\right\rangle}(s)=\frac{m_{c}^{2}\left\langle g_{s}^{2} G^{2}\right\rangle}{3 \cdot 2^{11} \pi^{6} s} \int_{\alpha_{\min }}^{\alpha_{\max }} \frac{d \alpha}{\alpha} \int_{\beta_{\min }}^{1-\alpha} \frac{d \beta}{\beta^{3}}(1-\alpha-\beta)\left\{(7 \alpha-3 \beta-3)\left[m_{c}^{2}(\alpha+\beta)-\alpha \beta s\right]-m_{c}^{2} \alpha(1-\alpha-\beta)\right\}
$$

(d.1) Condensado Misto $1 \quad D=5$
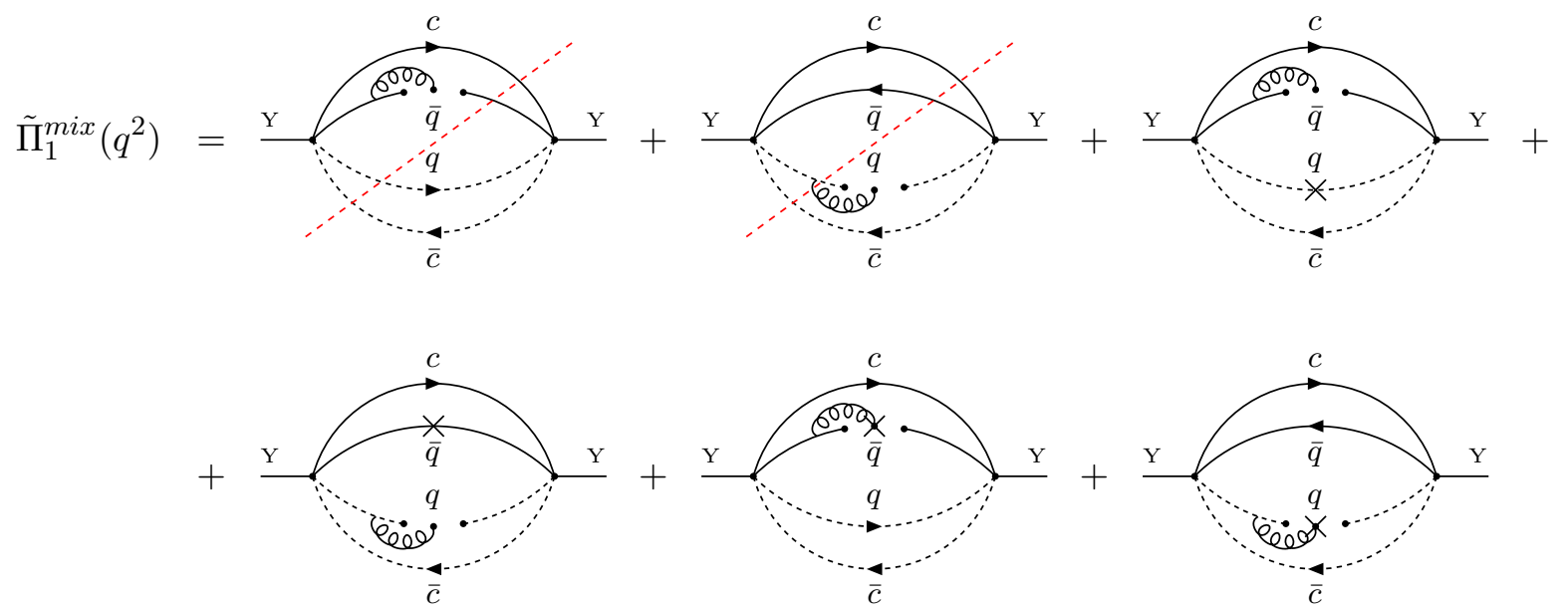

A contribuição não-perturbativa do condensado misto para a densidade espectral é dada por:

$$
\begin{aligned}
& \tilde{\rho}_{1}^{\text {mix }}(s)=\frac{3}{4} \rho_{1}^{\text {mix }}(s) \\
& \tilde{\rho}_{1}^{\text {mix }}(s)=-\frac{m_{q}\left\langle\bar{q} g_{s} \sigma G q\right\rangle}{2^{7} \pi^{4} s} \int_{\alpha_{\min }}^{\alpha_{\max }} d \alpha\left[5 m_{c}^{2}-\alpha(1-\alpha) s\right] .
\end{aligned}
$$

Esta é a contribuição onde o glúon no condensado misto é emitido pelo quark $q$ (ou antiquark $\bar{q}$ ) leve. O caso em que o glúon é emitido pelo quark $c$ (ou antiquark $\bar{c}$ ) pesado será analisado na sequência. 
(d.2) Condensado Misto $2 \quad D=5$

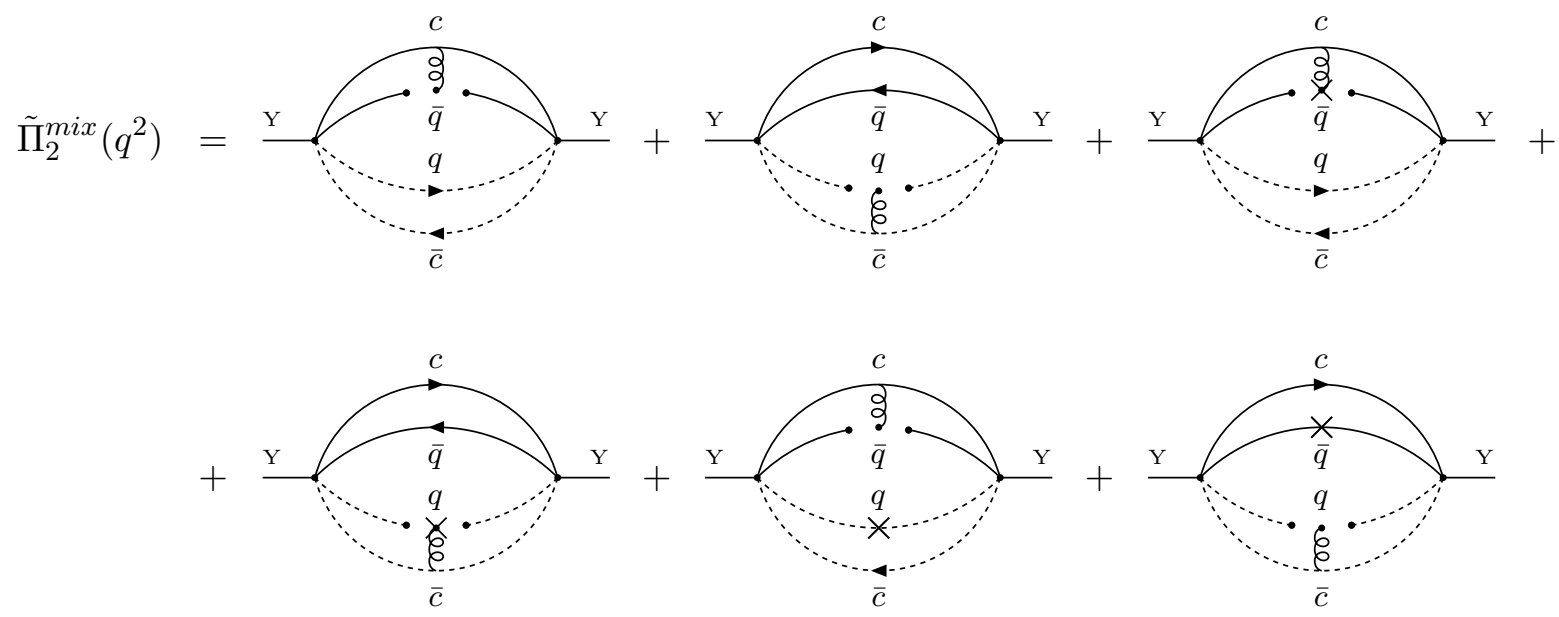

A contribuição não-fatorizável do condensado misto para a densidade espectral é dada por:

$$
\begin{aligned}
& \tilde{\rho}_{2}^{\text {mix }}(s)= \frac{3}{2} \rho_{2}^{\text {mix }}(s) \\
& \tilde{\rho}_{2}^{\text {mix }}(s)=\frac{\left\langle\bar{q} g_{s} \sigma G q\right\rangle}{2^{6} \pi^{4} s}\left\{\int_{\alpha_{\min }}^{\alpha_{\max }} \frac{d \alpha}{\alpha} \int_{\beta_{\min }}^{1-\alpha} \frac{d \beta}{\beta^{2}}\left[m_{c}(2 \alpha+\beta)\left(m_{c}^{2}(\alpha+\beta)-\alpha \beta s\right)+5 m_{q} m_{c}^{2} \alpha \beta\right]+\right. \\
&\left.\quad+m_{q} \int_{\alpha_{\min }}^{\alpha_{\max }} \frac{d \alpha}{(1-\alpha)}\left[m_{c}^{2}-\alpha(1-\alpha) s\right]\right\}
\end{aligned}
$$

(e) Condensado de 4-Quarks $D=6$

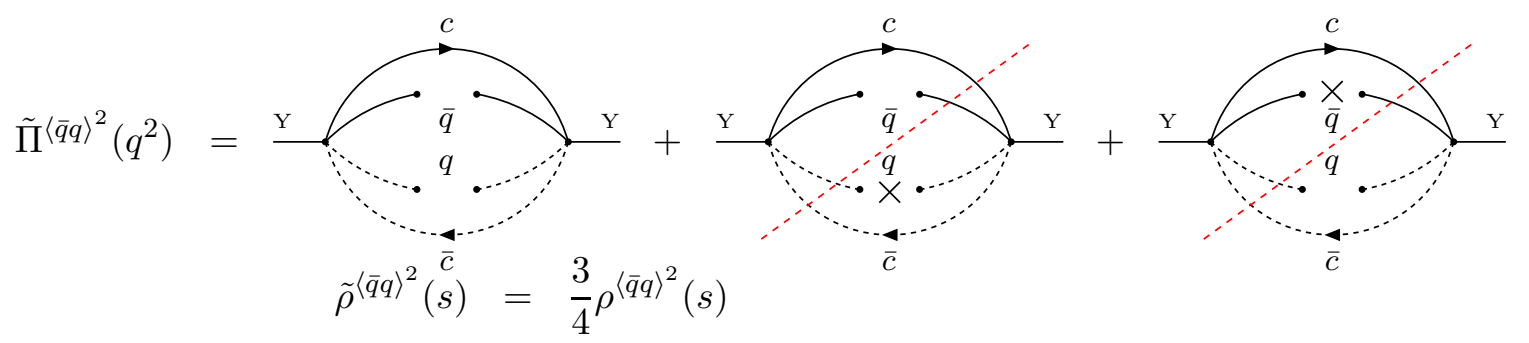

$$
\begin{aligned}
& \tilde{\rho}^{\langle\bar{q} q\rangle^{2}}(s)=-\frac{\langle\bar{q} q\rangle^{2}}{3 \cdot 2^{4} \pi^{2}}\left(\frac{5 m_{c}^{2}}{s}-\frac{1}{2}\right) \sqrt{1-4 m_{c}^{2} / s} .
\end{aligned}
$$

Obtidas as expressões das densidades espectrais, podemos prosseguir no cálculo dos parâmetros hadrônicos e igualar os dois lados da regra de soma.

\subsubsection{Dualidade Quark-Hadron}

Novamente temos a seguinte imposição à variável $s$ :

$$
s_{\min }=4 m_{c}^{2} .
$$


Como não foram utilizadas as contribuições dos condensados de dimensão 8, podemos utilizar a expressão (2.78) para obter a massa do estado molecular. Logo:

$$
\tilde{m}_{Y}^{2}=\frac{\int_{4 m_{c}^{2}}^{s_{0}} d s s \tilde{\rho}(s) e^{-s / M^{2}}}{\int_{4 m_{c}^{2}}^{s_{0}} d s \tilde{\rho}(s) e^{-s / M^{2}}}
$$

\subsubsection{Resultados Numéricos}

Para o cálculo numérico utilizamos os mesmos valores dados em (3.30). Analisamos as duas possíveis moléculas que podem ser descritas pela corrente (3.40).

\section{(a) Molécula com Estranheza}

Ao inserimos o quark estranho $s$ na corrente (3.40), o seu conteúdo de quarks fica dado por: $[c \bar{s}][s \bar{c}] \equiv D_{s 0} \bar{D}_{s}^{*}$. Logo, temos uma molécula com estranheza.

Da Figura (3.7), obtemos o limite superior para $M_{\max }^{2}$ em que $\sqrt{s_{0}}=4.9 \mathrm{GeV}$. Neste caso, temos $M_{\max }^{2} \leq 3.7 \mathrm{GeV}^{2}$.

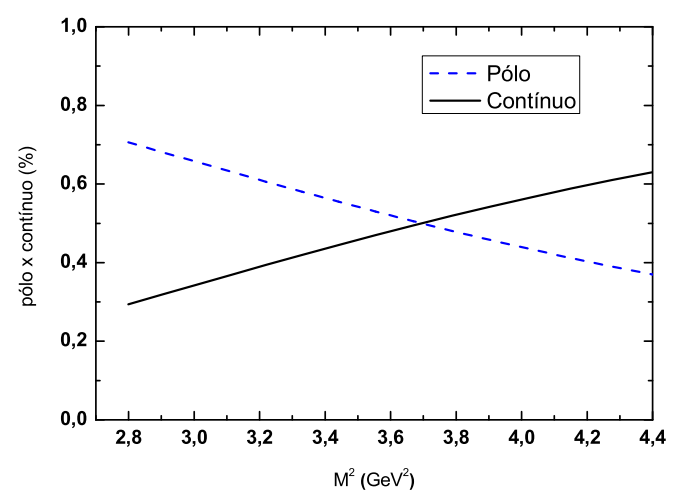

Figura 3.7: Relação Pólo x Contínuo, para $\sqrt{s_{0}}=4.9 \mathrm{GeV}$.

Para esta corrente, observamos que um intervalo apropriado para o limiar do contínuo é dado por $4.8 \leq \sqrt{s_{0}} \leq 5.0 \mathrm{GeV}$. Logo, escolhendo três valores de $\sqrt{s_{0}}$ neste intervalo, os respectivos limites superiores obtidos para $M^{2}$ são listados na tabela abaixo:

Tabela III: Limites superiores da Janela de Borel para as QCDSR da molécula $D_{s 0} \bar{D}_{s}^{*}$.

\begin{tabular}{|c|c|}
\hline$\sqrt{s_{0}}(\mathrm{GeV})$ & $M_{\max }^{2}\left(\mathrm{GeV}^{2}\right)$ \\
\hline 4.8 & 3.5 \\
\hline 4.9 & 3.7 \\
\hline 5.0 & 4.0 \\
\hline
\end{tabular}

O limite inferior de $M^{2}$ é determinado a partir da Figura (3.8). Obtemos uma boa convergência da OPE para $M_{\text {min }}^{2} \geq 3.2 \mathrm{GeV}^{2}$.

Portanto, a janela de Borel desta QCDSR é dada por: $3.2 \leq M^{2} \leq 4.0 \mathrm{GeV}^{2}$; e no intervalo de $s_{0}: 4.8 \leq \sqrt{s_{0}} \leq 5.0 \mathrm{GeV}$. 


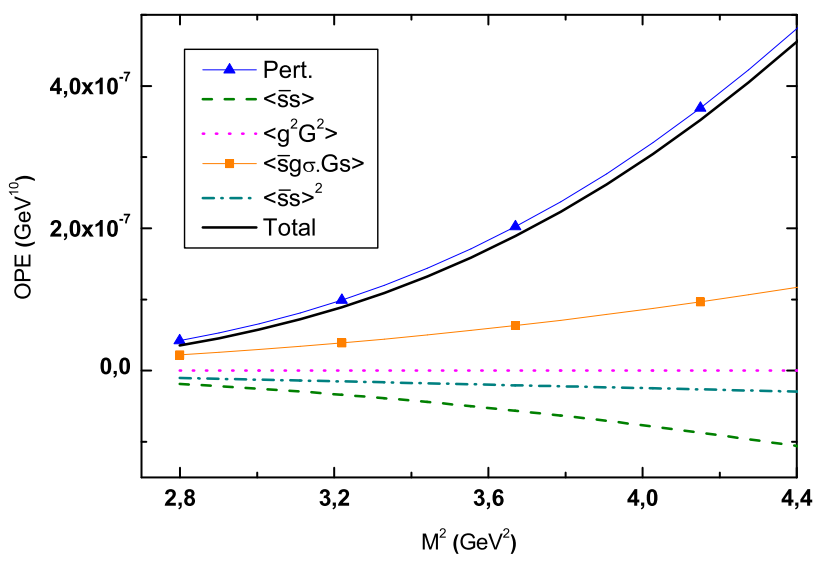

Figura 3.8: A convergência da OPE utilizando $\sqrt{s_{0}}=4.9 \mathrm{GeV}$.

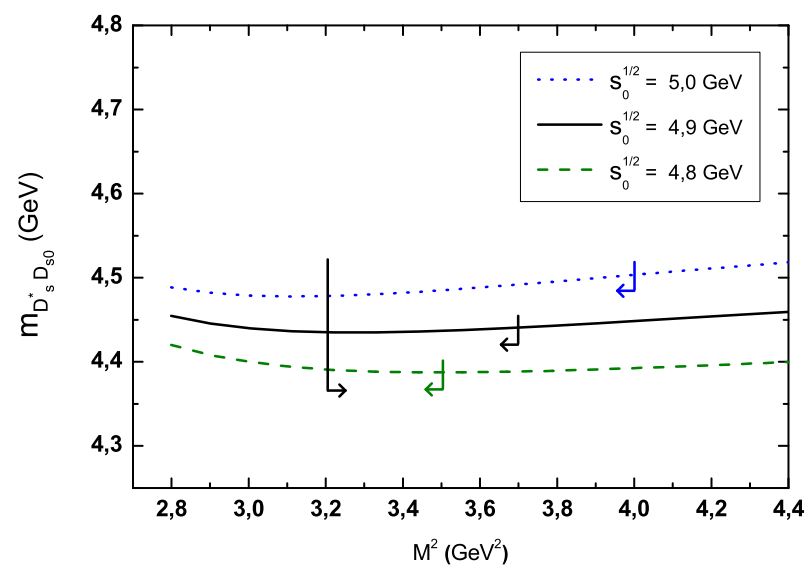

Figura 3.9: A massa da Molécula $D_{s 0} \bar{D}_{s}^{*}$ em função do parâmetro $M^{2}$, para diferentes valores de $\sqrt{s_{0}}$. As setas delimitam a janela de Borel das QCDSR.

Na Figura (3.9), é mostrada a massa da molécula para diferentes valores de $s_{0}$. Observamos, também, que há boa estabilidade de $\tilde{m}_{Y}$ dentro da janela de Borel.

Levando em consideração as variações de $M^{2}, s_{0},\langle\bar{q} q\rangle, m_{s}$ e $m_{c}$ dentro da janela de Borel, a massa da partícula correspondente a molécula $D_{s 0} \bar{D}_{s}^{*}$ é dada por:

$$
\tilde{m}_{Y}=(4.42 \pm 0.10) \mathrm{GeV}
$$

que é mais compatível com o $Y(4360)$ do que com os outros mésons. É importante mencionar que mesmo utilizando $\sqrt{s_{0}}=5.1 \mathrm{GeV}$, que foi o valor central usado para estudar o $Y(4660)$, o valor obtido para massa foi de $\tilde{m}_{Y} \approx 4.5 \mathrm{GeV}$. Isto mostra que ainda temos um resultado compatível com 3.57. Logo, concluímos que o méson $Y$ (4660) é melhor explicado com a corrente de tetraquark (3.1) do que com a corrente molecular (3.40).

Contudo, antes de associarmos o estado molecular ao méson $Y(4360)$, é necessário entendermos melhor o espectro de massa do dipíon dentro do decaimento $Y(4360) \longrightarrow \psi(2 S) \pi^{+} \pi^{-}$. Do espectro dado na ref. [32], o méson $Y(4360)$ é mais consistente com um estado em que não há estranheza. Portanto, apesar dos resultados obtidos com as QCDSR mostrarem que o $Y(4360)$ poder ser descrito por um estado molecular $D_{s 0} \bar{D}_{s}^{*}$, nos parece mais razoável associá-lo 
ao tetraquark com quark leve, por ser mais compatível com o espectro de massa do dipíon.

\section{(b) Molécula com Quark Leve}

Ao inserimos o quark $q=u, d$ na corrente (3.40), seu conteúdo de quarks fica dado por: $[c \bar{q}][q \bar{c}] \equiv D_{0} \bar{D}^{*}$. Da Figura (3.10), obtemos o limite superior para $M^{2}$ em que $\sqrt{s_{0}}=4.7 \mathrm{GeV}$. Neste caso, temos $M^{2} \leq 3.5 \mathrm{GeV}^{2}$.

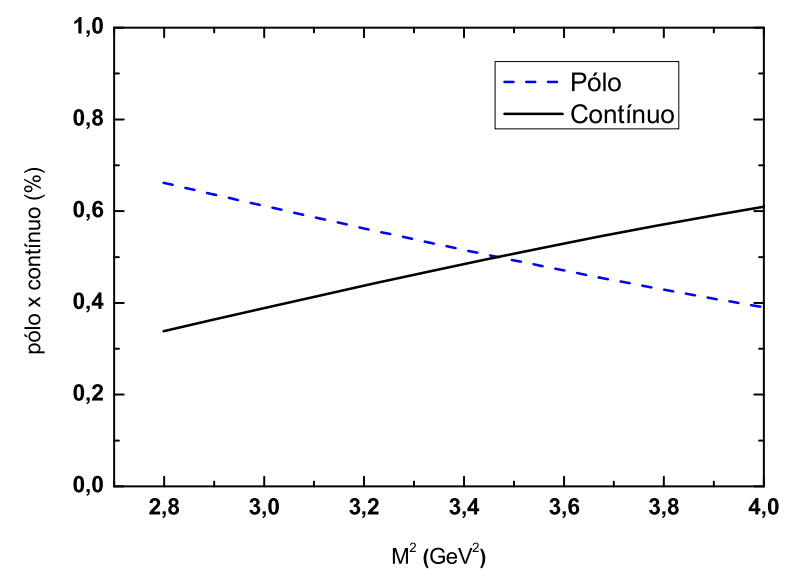

Figura 3.10: Relação Pólo x Contínuo, para $\sqrt{s_{0}}=4.7 \mathrm{GeV}$.

Para esta corrente, o intervalo apropriado para o limiar do contínuo é dado por $4.6 \leq \sqrt{s_{0}} \leq$ $4.8 \mathrm{GeV}$. Logo, escolhendo três valores de $\sqrt{s_{0}}$ neste intervalo, os respectivos limites superiores obtidos para $M^{2}$ são listados na tabela abaixo:

Tabela IV: Limites superiores da Janela de Borel para as QCDSR da molécula $D_{0} \bar{D}^{*}$.

\begin{tabular}{|c|c|}
\hline$\sqrt{s_{0}}(\mathrm{GeV})$ & $M_{\max }^{2}\left(\mathrm{GeV}^{2}\right)$ \\
\hline 4.6 & 3.3 \\
\hline 4.7 & 3.5 \\
\hline 4.8 & 3.7 \\
\hline
\end{tabular}

O limite inferior de $M^{2}$ é determinado a partir da Figura (3.11). Obtemos uma boa convergência da OPE para $M^{2} \geq 3.2 \mathrm{GeV}^{2}$.

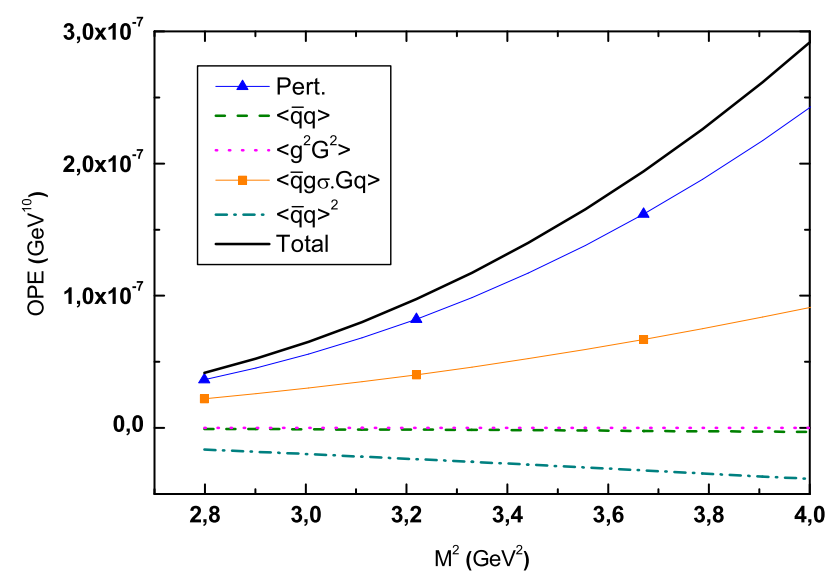

Figura 3.11: A convergência da OPE utilizando $\sqrt{s_{0}}=4.7 \mathrm{GeV}$. 
Portanto, a janela de Borel desta QCDSR é dada por: $3.2 \leq M^{2} \leq 3.7 \mathrm{GeV}^{2}$; e no intervalo de $s_{0}: 4.6 \leq \sqrt{s_{0}} \leq 4.8 \mathrm{GeV}$.

Na Figura (3.12), é mostrada a massa da molécula para diferentes valores de $s_{0}$. Há boa estabilidade de $\tilde{m}_{Y}$ dentro da janela de Borel.

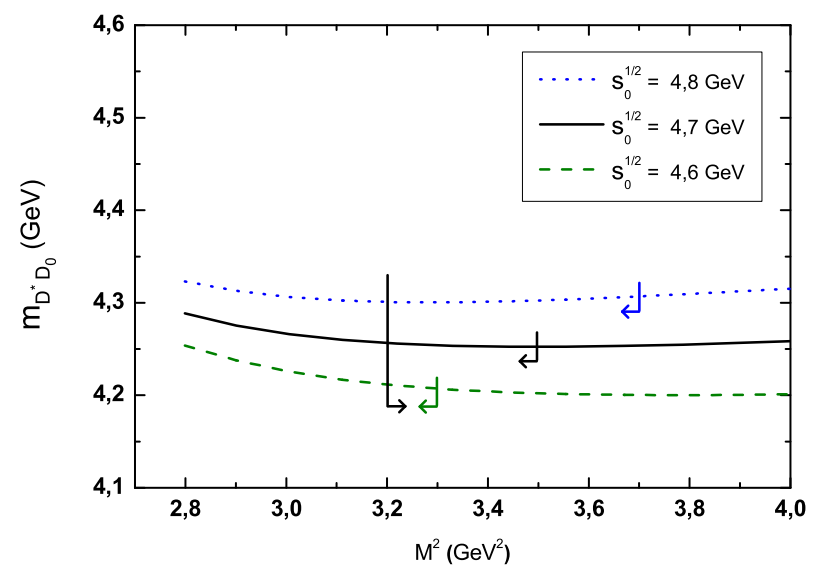

Figura 3.12: A massa da Molécula $D_{0} \bar{D}^{*}$ em função do parâmetro $M^{2}$, para diferentes valores de $\sqrt{s_{0}}$. As setas delimitam a janela de Borel das QCDSR.

Levando em consideração as variações de $M^{2}, s_{0},\langle\bar{q} q\rangle$ e $m_{c}$ dentro da janela de Borel, a massa da partícula correspondente a molécula $D_{0} \bar{D}^{*}$ é dada por:

$$
\tilde{m}_{Y}=(4.27 \pm 0.10) \mathrm{GeV}
$$

que está em excelente acordo com a massa do méson $Y(4260)$. Novamente, para concluir se nós podemos associar este estado molecular com o méson $Y(4260)$ é necessário analisar o espectro de massa do dipíon no decaimento $Y(4260) \longrightarrow J / \psi \pi^{+} \pi^{-}$. Do espectro dado na ref. [32], concluímos que o méson $Y(4260)$ é consistente com um estado molecular com quarks leves. 


\section{Capítulo 4}

\section{Conclusões}

A análise das Regras de Soma da QCD sobre a função de correlação de dois pontos dos estados exóticos com $J^{P C}=1^{--}$, revelaram alguns resultados interessantes para a família de mésons Y observada pelas colaborações BaBar e Belle. Consideramos dois tipos de correntes:

- um estado tetraquark formado pelo par diquark-antidiquark com uma distribuição simétrica de spin $[c q]_{S=1}[\bar{c} \bar{q}]_{S=0}+[c q]_{S=0}[\bar{c} \bar{q}]_{S=1}$ (onde o quark $q$ pode ser tanto os quarks $u$ e $d$ que formam o estado de tetraquark leve, como o quark estranho $s$ que forma o estado de tetraquark com estranheza);

- e um estado molecular com uma combinação simétrica entre os mésons charmosos escalares e vetoriais.

Nossos resultados indicam que o méson $Y(4660)$ pode ser descrito por um estado de tetraquark com estranheza $(c s \bar{c} \bar{s})$. Este conteúdo de quarks é consistente com o espectro de massa invariante do dipíon mostrado em [32], que mostra que há uma indicação de que o dipíon do decaimento do $Y(4660)$ é consistente com o estado intermediário $f_{0}(980)$.

No caso do méson $Y(4360)$, sua massa pode ser obtida, considerando os erros, se assumirmos que ele é constituído ou de um estado de tetraquark leve $[c q \bar{c} \bar{q}]$ ou de uma molécula $D_{s 0} \bar{D}^{*}$. Do espectro de massa invariante do dipion, observamos que este estado não é compatível com o estado intermediário $f_{0}(980)$, indicando que possivelmente não haja quarks $s$ na estrutura interna do méson $Y(4360)$, o que favorece a estrutura de tetraquark $[c q \bar{c} \bar{q}]$ para o méson $Y(4360)$. No entanto, os resultados com a molécula $D_{s 0} \bar{D}^{*}$ implica que ainda é necessário uma melhor análise deste estado antes de tentarmos associá-lo a qualquer tipo de estrutura (tetraquark ou molécula).

De acordo com os nossos resultados, o méson $Y(4260)$ é muito bem descrito por um estado molecular $D_{0} \bar{D}^{*}$.

Os resultados obtidos neste trabalho foram publicados no periódico Nuclear Physics $A$ e encontram-se na referência [51]. 



\section{Apêndice A}

\section{Propagadores Utilizados nas QCDSR}

Ao longo deste trabalho os condensados do lado da QCD foram obtidos pela aplicação do teorema de Wick. Este procedimento expande um produto temporalmente ordenado de operadores de campo em função do produto dos propagadores associados a estes propagadores:

$$
\begin{aligned}
T\left[q_{a}(x) q_{b}(y) \bar{q}_{a}(x) \bar{q}_{b}(y)\right]= & : q_{a}(x) q_{b}(y) \bar{q}_{a}(x) \bar{q}_{b}(y):+q_{a}(x) \bar{q}_{b}(y): S_{a b}^{q}(y-x)+ \\
& +: q_{b}(y) \bar{q}_{a}(x): S_{a b}^{q}(x-y)+S_{a b}^{q}(y-x) S_{a b}^{q}(x-y)
\end{aligned}
$$

onde $S_{a b}^{q}(x-y)$ são os propagadores dos quarks livres.

Em uma teoria perturbativa, o valor esperado no vácuo do produto normal $: q_{a}(x) \bar{q}_{b}(y)$ : seria nulo, pois estaríamos criando e aniquilando, sucessivamente, férmions neste vácuo. Já em uma teoria não-perturbativa, são justamente estes termos que nos fornecem alguma informação sobre a dinâmica de processos de baixas energias.

Podemos lidar com estes produtos normais incluindo-os na expressão do propagador da seguinte forma:

$$
S_{a b}=S_{a b}^{0}(x)+\left\langle 0\left|: q_{a}(x) \bar{q}_{b}(0):\right| 0\right\rangle
$$

onde $S_{a b}^{0}(x)$ é o propagador perturbativo usual. Listaremos a seguir os propagadores pertubativos, não-perturbativos e não-fatorizáveis utilizados neste trabalho. 


\section{A.1 Propagadores dos Quarks Leves}

Em termos de diagramas, os propagadores dos quarks leves $(q=u, d, s)$ são dados por:

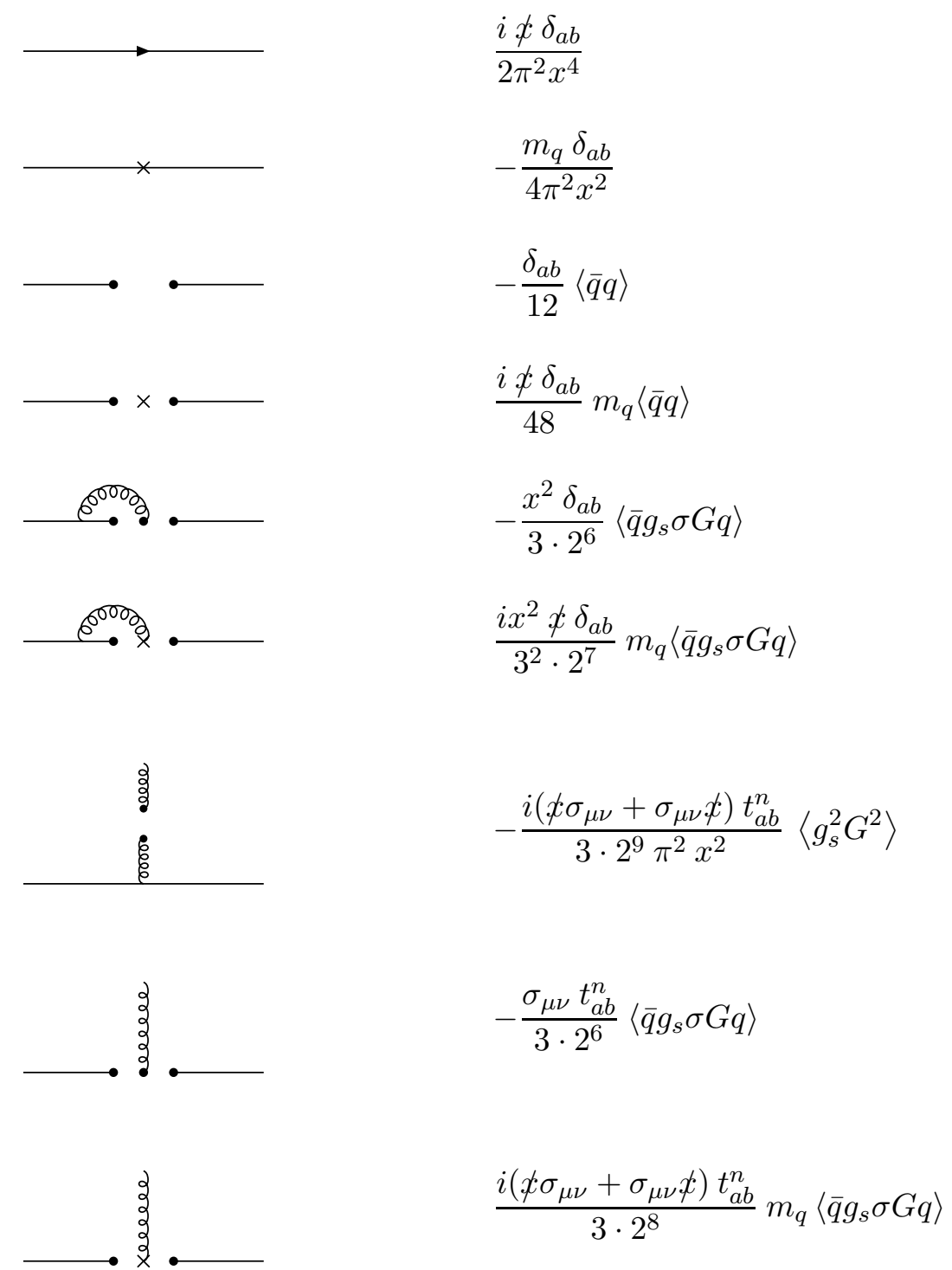

Os três últimos diagramas (não-fatoráveis) devem ser tratados com cuidado, pois devem sempre aparecer associados a um termo pertubativo de glúons em outro propagador, no qual devemos omitir $g_{s} G_{a b}^{n}(0)$. 


\section{A.2 Propagadores dos Quarks Pesados}

No caso de quarks pesados o condensado de quarks é muito pequeno e pode ser desprezado. Restam apenas os condensados de glúons gerados pela expressão (2.18) e por outros termos da expansão (2.15). Como não podemos expandir as expressões para massa pequena do quark, fica muito complicado passar para o espaço das coordenadas e o cálculo acaba sendo realizado no espaço dos momentos. As expressões utilizadas neste trabalho para estas contribuições e seus respectivos diagramas são [40]:

$$
\begin{aligned}
& \frac{i\left(\not p+m_{c}\right) \delta_{a b}}{p^{2}-m_{c}^{2}} \\
& -\frac{i t_{a b}^{n} g_{s} G_{\mu \nu}^{n}(0)}{4}\left[\frac{\not p \sigma^{\mu \nu}+\sigma^{\mu \nu} \not p+2 m_{c} \sigma^{\mu \nu}}{\left(p^{2}-m_{c}^{2}\right)^{2}}\right] \\
& \frac{i \delta_{a b}}{12}\left[\frac{p^{2}+m_{c} \not p}{\left(p^{2}-m_{c}^{2}\right)^{4}}\right] m_{c}\left\langle g_{s}^{2} G^{2}\right\rangle
\end{aligned}
$$





\section{Apêndice B}

\section{Fórmulas Úteis para as QCDSR}

\section{B.1 Transformadas de Fourier}

Algumas transformadas utilizadas no Capítulo 2:

$$
\begin{aligned}
\int d^{4} p \frac{e^{i p x}}{p^{2}+i \epsilon} & =\frac{i 4 \pi^{2}}{x^{2}} \\
\int d^{4} p \frac{\not p e^{i p x}}{p^{2}+i \epsilon} & =\frac{8 \pi^{2} \not x}{x^{4}} \\
\int d^{4} p \frac{\not p e^{i p x}}{\left(p^{2}+i \epsilon\right)^{2}} & =\frac{2 \pi^{2} \not x}{x^{2}} .
\end{aligned}
$$

\section{B.2 Álgebra das Matrizes de Gell-Mann}

Destacamos as seguintes propriedades destas matrizes:

$$
\begin{aligned}
t_{a a}^{n} & =0 \\
t_{a b}^{n} t_{b a}^{m} & =\frac{\delta_{n m}}{2} \\
t_{a b}^{n} t_{c d}^{n} & =\frac{1}{2}\left(\delta_{a d} \delta_{b c}-\frac{1}{3} \delta_{a b} \delta_{c d}\right) .
\end{aligned}
$$

\section{B.3 Álgebra das Matrizes de Dirac}

As matrizes de Dirac, na representação de Pauli, são dadas por:

$$
\gamma^{0}=\left(\begin{array}{cc}
\mathbb{1} & 0 \\
0 & -\mathbb{1}
\end{array}\right), \quad \gamma^{i}=\left(\begin{array}{cc}
0 & \sigma^{\mathbf{i}} \\
-\sigma^{\mathbf{i}} & 0
\end{array}\right)
$$

onde $\sigma^{\mathbf{i}}$ são as matrizes de Pauli. E satisfazem as propriedades: 


$$
\begin{aligned}
\left\{\gamma_{\mu}, \gamma_{\nu}\right\} & =2 g_{\mu \nu} \\
\gamma_{\mu} \gamma^{\mu} & =4 \\
\gamma^{0}=\gamma^{0 T} & =\gamma^{0 \dagger} \\
\gamma^{0} \gamma^{\mu} \gamma^{0 \dagger} & =\gamma^{\mu \dagger}
\end{aligned}
$$

Dadas as definições:

$$
\begin{aligned}
C & =i \gamma^{2} \gamma^{0} \\
\sigma_{\mu \nu} & =i\left(\gamma_{\mu} \gamma_{\nu}-g_{\mu \nu}\right) \\
\gamma_{5} & =i \gamma^{0} \gamma^{1} \gamma^{2} \gamma^{3} \\
K_{\mu \nu}(x) & =\not \sigma_{\mu \nu}+\sigma_{\mu \nu} \not .
\end{aligned}
$$

Obtemos as seguintes propriedades:

$$
\begin{aligned}
C^{-1}=C^{T} & =C^{\dagger}=-C \\
C \gamma_{\mu}^{T} C^{-1} & =-\gamma_{\mu} \\
C \sigma_{\mu \nu}^{T} C^{-1} & =-\sigma_{\mu \nu} \\
C K_{\mu \nu}^{T}(x) C^{-1} & =K_{\mu \nu}(x) \\
K^{2}(x) & =24 x^{2} \\
\sigma^{\mu \nu} \sigma_{\mu \nu} & =12 \\
\gamma_{5}=\gamma_{5}^{T} & =\gamma_{5}^{\dagger} \\
\left\{\gamma^{\mu}, \gamma_{5}\right\} & =0 \\
\sigma_{\mu \nu} \gamma^{\alpha} \sigma^{\mu \nu} & =0 \\
\gamma_{\mu} \gamma^{\alpha} \gamma^{\mu} & =-2 \gamma^{\alpha} \\
\sigma_{\alpha \beta}\left(\gamma_{\mu} \gamma_{\nu}\right) \sigma^{\alpha \beta} & =16 g_{\mu \nu}-4 \gamma_{\mu} \gamma_{\nu} .
\end{aligned}
$$

\section{B.4 Cálculo dos Traços}

É importante destacar algumas relações que facilitam o cálculo dos traços que surgem na função de correlação:

$$
\begin{aligned}
\operatorname{Tr}[\mathbf{1}] & =4 \\
\operatorname{Tr}\left[\gamma_{5}\right] & =0 \\
\text { O Traço de um } n^{o} \text { ímpar de } \gamma^{\prime s} & =0 \\
\operatorname{Tr}\left[\gamma_{\mu} \gamma_{\nu}\right] & =4 g_{\mu \nu} \\
\operatorname{Tr}\left[\gamma_{\mu} \gamma_{\nu} \gamma_{5}\right] & =0 \\
\operatorname{Tr}\left[\gamma_{\mu} \gamma_{\nu} \gamma_{\rho} \gamma_{\sigma}\right] & =4\left(g_{\mu \nu} g_{\rho \sigma}-g_{\mu \rho} g_{\nu \sigma}+g_{\mu \sigma} g_{\nu \rho}\right) \\
\operatorname{Tr}\left[\sigma_{\mu \nu}\right] & =0 .
\end{aligned}
$$




\section{B.5 Outras Relações Importantes}

- Contração de Cor para os Tetraquarks:

$$
\begin{aligned}
& \sum \epsilon_{a b c} \epsilon_{d e c} \epsilon_{i j k} \epsilon_{l m k} \cdot \delta_{b i} \delta_{a j} \delta_{d m} \delta_{e l}=12 \\
& \sum \epsilon_{a b c} \epsilon_{d e c} \epsilon_{i j k} \epsilon_{l m k} \cdot \delta_{b i} \delta_{a j} t_{d m}^{n} t_{e l}^{n}=-8
\end{aligned}
$$

- Contração de Cor para as Moléculas:

$$
\begin{aligned}
& \sum \delta_{a i} \delta_{i a} \delta_{b j} \delta_{j b}=9 \\
& \sum t_{a i} t_{i a} \delta_{b j} \delta_{j b}=12
\end{aligned}
$$

- Densidade Espectral:

$$
\begin{aligned}
\rho(s) & =\frac{1}{\pi} \operatorname{Im}[\Pi(s)] \\
\operatorname{Im}[\ln f(s)] & =-\pi, \quad \text { para } f(s)<0
\end{aligned}
$$

- Parâmetro de Schwinger $(\alpha)$ :

$$
\frac{1}{\left(m^{2}-p^{2}\right)^{n}}=\frac{1}{\Gamma(n)} \int_{0}^{\infty} d \alpha \alpha^{n-1} e^{-\alpha\left(m^{2}-p^{2}\right)}
$$

- Rotação de Wick:

$$
\begin{gathered}
d^{4} p=i d^{4} p_{e} \text { e } p^{2}=-p_{e}^{2} \\
d^{4} x=-i d^{4} x_{e} \text { e } x^{2}=-x_{e}^{2}
\end{gathered}
$$

- Integrais Gaussianas:

$$
\begin{gathered}
\int_{-\infty}^{+\infty} d^{4} p_{e} e^{-a p_{e}^{2}+x p_{e}}=\left(\frac{\pi}{a}\right)^{2} e^{\frac{x^{2}}{4 a}} \\
\int_{-\infty}^{+\infty} d^{4} x_{e} e^{-a x_{e}^{2}}=\frac{\pi^{2}}{a^{2}}
\end{gathered}
$$




\section{B.6 Resolução das Integrais}

\section{B.6.1 Integrais no Momento $p$}

Durante o desenvolvimento das funções de correlação, precisamos resolver integrais no momento do tipo:

$$
\int d^{4} p \frac{e^{-i p x}}{\left(p^{2}-m^{2}\right)^{n}} \quad \text { e } \quad \int d^{4} p \frac{p_{\mu} e^{-i p x}}{\left(p^{2}-m^{2}\right)^{n}}
$$

Os resultados destas integrais são obtidas da seguinte forma:

- Primeira integral: utilizando a Eq. (B.36)

$$
\begin{aligned}
\int d^{4} p \frac{e^{-i p x}}{\left(p^{2}-m^{2}\right)^{n}} & =(-1)^{n} \int d^{4} p \frac{e^{-i p x}}{\left(m^{2}-p^{2}\right)^{n}} \\
& =(-1)^{n} \int d^{4} p e^{-i p x} \int_{0}^{\infty} d \alpha \frac{\alpha^{n-1}}{\Gamma(n)} e^{-\alpha\left(m^{2}-p^{2}\right)} .
\end{aligned}
$$

Invertendo as integrais e indo para o espaço euclidiano, via rotação de Wick (B.37):

$$
\begin{aligned}
\int d^{4} p \frac{e^{-i p x}}{\left(p^{2}-m^{2}\right)^{n}} & =(-1)^{n} \int_{0}^{\infty} d \alpha \frac{\alpha^{n-1}}{\Gamma(n)} e^{-\alpha m^{2}} \int d^{4} p e^{\alpha p^{2}-i p x} \\
& =\frac{(-1)^{n} i}{\Gamma(n)} \int_{0}^{\infty} d \alpha \alpha^{n-1} e^{-m^{2} \alpha} \int d^{4} p_{e} e^{-\alpha p_{e}^{2}+x p_{e}}
\end{aligned}
$$

Utilizando a integral gaussiana (B.39), obtemos:

$$
\int d^{4} p \frac{e^{-i p x}}{\left(p^{2}-m^{2}\right)^{n}}=\frac{(-1)^{n} i \pi^{2}}{\Gamma(n)} \int_{0}^{\infty} \frac{d \alpha}{\alpha^{3-n}} e^{-\alpha m^{2}+\frac{x^{2}}{4 \alpha}} .
$$

- Segunda integral: utilizando a Eq. (B.36)

$$
\begin{aligned}
\int d^{4} p \frac{p_{\mu} e^{-i p x}}{\left(p^{2}-m^{2}\right)^{n}} & =(-1)^{n} \int d^{4} p \frac{p_{\mu} e^{-i p x}}{\left(m^{2}-p^{2}\right)^{n}} \\
& =(-1)^{n} \int d^{4} p p_{\mu} e^{-i p x} \int_{0}^{\infty} d \beta \frac{\beta^{n-1}}{\Gamma(n)} e^{-\beta\left(m^{2}-p^{2}\right)} .
\end{aligned}
$$

A integral no momento pode ser reescrita em termos de uma derivada:

$$
\int d^{4} p \frac{p_{\mu} e^{-i p x}}{\left(p^{2}-m^{2}\right)^{n}}=\frac{(-1)^{n} i}{\Gamma(n)} \int_{0}^{\infty} d \beta \beta^{n-1} e^{-\beta m^{2}} \frac{\partial}{\partial x_{\mu}}\left[\int d^{4} p e^{\beta p^{2}-i p x}\right]
$$

que via rotação de Wick se transforma em uma integral gaussiana (B.39):

$$
\begin{aligned}
\int d^{4} p \frac{p_{\mu} e^{-i p x}}{\left(p^{2}-m^{2}\right)^{n}} & =\frac{(-1)^{n+1}}{\Gamma(n)} \int_{0}^{\infty} d \beta \beta^{n-1} e^{-\beta m^{2}} \frac{\partial}{\partial x_{\mu}}\left[\int d^{4} p_{e} e^{-\beta p_{e}^{2}+x p_{e}}\right] \\
& =\frac{(-1)^{n+1} \pi^{2}}{2 \Gamma(n)} \int_{0}^{\infty} \frac{d \beta}{\beta^{3-n}} e^{-\beta m^{2}} \frac{\partial}{\partial x_{\mu}}\left[e^{\frac{x^{2}}{4 \beta}}\right] .
\end{aligned}
$$


Finalmente, obtemos:

$$
\int d^{4} p \frac{p_{\mu} e^{-i p x}}{\left(p^{2}-m^{2}\right)^{n}}=\frac{(-1)^{n+1} \pi^{2}}{2 \Gamma(n)} \int_{0}^{\infty} \frac{d \beta}{\beta^{4-n}} x_{\mu} e^{-\beta m^{2}+\frac{x^{2}}{4 \beta}} .
$$

Portanto, as integrais no momento são dadas por:

$$
\begin{aligned}
& \int d^{4} p \frac{e^{-i p x}}{\left(p^{2}-m^{2}\right)^{n}}=\frac{(-1)^{n} i \pi^{2}}{\Gamma(n)} \int_{0}^{\infty} \frac{d \alpha}{\alpha^{3-n}} e^{-\alpha m^{2}+\frac{x^{2}}{4 \alpha}} \\
& \int d^{4} p \frac{p_{\mu} e^{-i p x}}{\left(p^{2}-m^{2}\right)^{n}}=\frac{(-1)^{n+1} \pi^{2}}{2 \Gamma(n)} \int_{0}^{\infty} \frac{d \beta}{\beta^{4-n}} x_{\mu} e^{-\beta m^{2}+\frac{x^{2}}{4 \beta}} .
\end{aligned}
$$

\section{B.6.2 Integrais na Coordenada $x$}

As integrais necessárias, obtidas através das integrais gaussianas, são $\left(Q^{2}=-q^{2}\right)$ :

$$
\begin{aligned}
\int d^{4} x e^{i q x+\frac{(\alpha+\beta) x^{2}}{4 \alpha \beta}} & =-\frac{i 2^{4} \pi^{2} \alpha^{2} \beta^{2}}{(\alpha+\beta)^{2}} e^{-\frac{\alpha \beta Q^{2}}{\alpha+\beta}} \\
\int d^{4} x x^{2} e^{i q x+\frac{(\alpha+\beta) x^{2}}{4 \alpha \beta}} & =\frac{i 2^{6} \pi^{2} \alpha^{3} \beta^{3}}{(\alpha+\beta)^{3}} e^{-\frac{\alpha \beta Q^{2}}{\alpha+\beta}} \cdot\left(2-\frac{\alpha \beta Q^{2}}{\alpha+\beta}\right) \\
\int d^{4} x x^{4} e^{i q x+\frac{(\alpha+\beta) x^{2}}{4 \alpha \beta}} & =-\frac{i 2^{8} \pi^{2} \alpha^{4} \beta^{4}}{(\alpha+\beta)^{4}} e^{-\frac{\alpha \beta Q^{2}}{\alpha+\beta}} \cdot\left[6-\frac{6 \alpha \beta Q^{2}}{\alpha+\beta}+\frac{\alpha^{2} \beta^{2} Q^{4}}{(\alpha+\beta)^{2}}\right] .
\end{aligned}
$$

\section{B.6.3 Integral $I_{n}$}

Dada a integral

$$
I_{n}=\int \frac{d^{4} x}{\left(x^{2}\right)^{n}} e^{i q x+\frac{(\alpha+\beta)}{4 \alpha \beta} x^{2}} .
$$

Para resolvê-la, devemos inicialmente ir para o espaço euclidiano e utilizando a Eq. (B.36):

$$
\begin{aligned}
I_{n} & =-i \int d^{4} x_{e} \frac{e^{-i Q x_{e}}}{\left(-x_{e}^{2}\right)^{n}} \\
& =\frac{(-1)^{n-1} i}{(n-1) !} \int d^{4} x_{e} e^{-i Q x_{e}-\left(\frac{1}{4 \alpha}+\frac{1}{4 \beta}\right) x_{e}^{2}} \int_{0}^{\infty} d \delta \delta^{n-1} e^{-\delta x_{e}^{2}} .
\end{aligned}
$$

Definindo a variável $b=\frac{1}{4 \alpha}+\frac{1}{4 \beta}+\delta$ e completando o quadrado do termo da exponencial 
para utilizar a integral gaussiana (B.40):

$$
\begin{aligned}
I_{n} & =\frac{(-1)^{n-1} i}{(n-1) !} \int_{0}^{\infty} d \delta \delta^{n-1} e^{-\frac{Q^{2}}{4 b}} \int d^{4} x_{e} e^{-b\left(x_{e}+\frac{i Q}{2 b}\right)^{2}} \\
& =\frac{(-1)^{n-1} i \pi^{2}}{(n-1) !} \int_{0}^{\infty} d \delta \frac{\delta^{n-1}}{b^{2}} e^{-\frac{Q^{2}}{4 b}}
\end{aligned}
$$

Por fim, fazendo a mudança de variáveis $\delta=\frac{1}{4 \gamma}$, obtemos:

$$
I_{n}=\frac{(-1)^{n-1} i \pi^{2}}{(n-1) ! 4^{n-2}} \int_{0}^{\infty} \frac{d \gamma}{\gamma^{n-1}} \frac{\alpha^{2} \beta^{2}}{(\alpha \beta+\beta \gamma+\gamma \alpha)^{2}} e^{-\frac{\alpha \beta \gamma Q^{2}}{\alpha \beta+\beta \gamma+\gamma \alpha}}
$$

\section{B.6.4 Integral $I_{n m l}$}

Dada a integral

$$
I_{n m l}=\int_{0}^{\infty} \frac{d \alpha d \beta}{\alpha^{n} \beta^{m}} \frac{e^{-m_{c}^{2}(\alpha+\beta)-\frac{\alpha \beta Q^{2}}{\alpha+\beta}}}{(\alpha+\beta)^{l}}
$$

Para resolvê-la, inserimos a identidade $1=\int_{0}^{\infty} d \lambda \delta[\lambda-(\alpha+\beta)]$ :

$$
\begin{aligned}
I_{n m l} & =\int_{0}^{\infty} \frac{d \alpha d \beta}{\alpha^{n} \beta^{m}} \frac{e^{-m_{c}^{2}(\alpha+\beta)-\frac{\alpha \beta Q^{2}}{\alpha+\beta}}}{(\alpha+\beta)^{l}} \\
& =\int_{0}^{\infty} \frac{d \alpha d \beta}{\alpha^{n} \beta^{m}} \frac{e^{-m_{c}^{2}(\alpha+\beta)-\frac{\alpha \beta Q^{2}}{\alpha+\beta}}}{(\alpha+\beta)^{l}} \int_{0}^{\infty} d \lambda \delta[\lambda-(\alpha+\beta)] .
\end{aligned}
$$

Fazendo a mudança de escala: $\alpha \rightarrow \lambda \alpha$ e $\beta \rightarrow \lambda \beta$ :

$$
I_{n m l}=\int_{0}^{\infty} \frac{d \alpha d \beta d \lambda}{\alpha^{n} \beta^{m} \lambda^{n+m+l-1}} \frac{e^{-m_{c}^{2} \lambda(\alpha+\beta)-\frac{\alpha \beta \lambda Q^{2}}{\alpha+\beta}}}{(\alpha+\beta)^{l}} \delta[1-(\alpha+\beta)] .
$$

Integrando em $\beta$ através da função delta, obtemos o resultado:

$$
I_{n m l}=\int_{0}^{1} \frac{d \alpha}{\alpha^{n}(1-\alpha)^{m}} \int_{0}^{\infty} \frac{d \lambda}{\lambda^{n+m+l-1}} e^{-\lambda\left[m_{c}^{2}+\alpha(1-\alpha) Q^{2}\right]} .
$$




\section{B.6.5 Integral $I_{n m k l}$}

Dada a integral

$$
I_{n m k l}=\int \frac{d \alpha d \beta d \gamma}{\alpha^{n} \beta^{m} \gamma^{k}} \frac{e^{-m_{c}^{2}(\alpha+\beta)-\frac{\alpha \beta \gamma Q^{2}}{\alpha \beta+\beta \gamma+\gamma \alpha}}}{(\alpha \beta+\beta \gamma+\gamma \alpha)^{l}} .
$$

Podemos reescrevê-la como:

$$
I_{n m k l}=\int \frac{d \alpha d \beta d \gamma}{\alpha^{n} \beta^{m} \gamma^{k+l}} \frac{e^{-m_{c}^{2}(\alpha+\beta)-\frac{\alpha \beta Q^{2}}{(\alpha \beta / \gamma)+\beta+\alpha}}}{\left(\frac{\alpha \beta}{\gamma}+\beta+\alpha\right)^{l}} .
$$

Assim, fazendo a mudança de variáveis: $\alpha=\alpha^{\prime}, \beta=\beta^{\prime}$ e $\frac{\alpha \beta}{\gamma}=\gamma^{\prime}$ :

$$
I_{n m k l}=\int \frac{d \alpha^{\prime} d \beta^{\prime} d \gamma^{\prime}}{\alpha^{\prime n+k+l-1} \beta^{\prime m+k+l-1}} \frac{\gamma^{\prime l+k-2}}{\left(\alpha^{\prime}+\beta^{\prime}+\gamma^{\prime}\right)^{l}} e^{-m_{c}^{2}\left(\alpha^{\prime}+\beta^{\prime}\right)-\frac{\alpha^{\prime} \beta^{\prime} Q^{2}}{\alpha^{\prime}+\beta^{\prime}+\gamma^{\prime}}}
$$

Omitindo os ' nas variáveis e inserindo a identidade $1=\int_{0}^{\infty} d \lambda \delta[\lambda-(\alpha+\beta+\gamma)]$ :

$$
I_{n m k l}=\int \frac{d \alpha d \beta d \gamma}{\alpha^{n+k+l-1} \beta^{m+k+l-1}} \frac{\gamma^{l+k-2}}{(\alpha+\beta+\gamma)^{l}} e^{-m_{c}^{2}(\alpha+\beta)-\frac{\alpha \beta Q^{2}}{\alpha+\beta+\gamma}} \int_{0}^{\infty} d \lambda \delta[\lambda-(\alpha+\beta+\gamma)]
$$

Fazendo a mudança de escala: $\alpha \rightarrow \lambda \alpha, \beta \rightarrow \lambda \beta$ e $\gamma \rightarrow \lambda \gamma$ :

$$
I_{n m k l}=\int \frac{d \alpha d \beta d \gamma d \lambda \delta[1-(\alpha+\beta+\gamma)]}{\alpha^{n+k+l-1} \beta^{m+k+l-1} \lambda^{n+m+k+2 l-2}} \frac{\gamma^{l+k-2}}{(\alpha+\beta+\gamma)^{l}} e^{-m_{c}^{2} \lambda(\alpha+\beta)-\frac{\alpha \beta \lambda Q^{2}}{\alpha+\beta+\gamma}} .
$$

Integrando em $\gamma$ através da função delta, obtemos o resultado:

$$
I_{n m k l}=\int_{0}^{1} \frac{d \alpha d \beta \theta(1-\alpha-\beta)}{\alpha^{n+k+l-1} \beta^{m+k+l-1}}(1-\alpha-\beta)^{l+k-2} \int_{0}^{\infty} d \lambda \frac{e^{-\lambda\left[m_{c}^{2}(\alpha+\beta)+\alpha \beta Q^{2}\right]}}{\lambda^{n+m+k+2 l-2}} .
$$

\section{B.6.6 Integral em $\lambda$}

Integrais do tipo

$$
\int \frac{d \lambda}{\lambda^{n}} e^{-\lambda f}
$$

onde $f$ é uma função positiva qualquer e $n$ a potência de $\lambda$, são obtidas começando pela seguinte integral:

$$
\int_{0}^{\infty} d \lambda e^{-\lambda f}=\frac{1}{f}
$$


Note que a integral acima pode ser reescrita por:

$$
\int_{0}^{\infty} d \lambda e^{-\lambda f}=-\frac{\partial}{\partial f} \int_{0}^{\infty} \frac{d \lambda}{\lambda} e^{-\lambda f} .
$$

Logo, integrando em $f$ em ambos os lados, obtemos o resultado para $n=1$ :

$$
\begin{aligned}
\int_{0}^{\infty} \frac{d \lambda}{\lambda} e^{-\lambda f} & =-\int d f \int_{0}^{\infty} d \lambda e^{-\lambda f} \\
& =-\int \frac{d f}{f}=-\ln f+\text { cte }
\end{aligned}
$$

A constante é um número real que carrega a divergência da integral. Não é necessário nos preocupar com ela pois nas QCDSR precisamos apenas da parte imaginária destas integrais. O passo seguinte $(n=2)$ é obtido de forma análoga:

$$
\int_{0}^{\infty} \frac{d \lambda}{\lambda^{2}} e^{-\lambda f}=\int d f \ln f=f \ln f-f+\text { cte }
$$

onde novamente só a parte $f(\ln f)$ interessa, pois é a única que pode ter parte imaginária. As integrais seguintes são obtidas da mesma forma:

$$
\begin{aligned}
& \int_{0}^{\infty} \frac{d \lambda}{\lambda^{3}} e^{-\lambda f}=-\frac{f^{2} \ln f}{2}+\cdots \\
& \int_{0}^{\infty} \frac{d \lambda}{\lambda^{4}} e^{-\lambda f}=\frac{f^{3} \ln f}{3 !}+\cdots \\
& \int_{0}^{\infty} \frac{d \lambda}{\lambda^{5}} e^{-\lambda f}=-\frac{f^{4} \ln f}{4 !}+\cdots
\end{aligned}
$$

por indução concluímos finalmente que:

$$
\int \frac{d \lambda}{\lambda^{n}} e^{-\lambda f}=\frac{(-1)^{n}}{(n-1) !} f^{n-1} \ln f+\mathcal{O}\left(f^{n}\right) .
$$




\section{Apêndice C}

\section{Cálculo dos Diagramas de Tetraquarks}

Nas seções seguintes mostraremos alguns exemplos detalhados do cálculo das contribuições: perturbativa, não-perturbativa e não-fatorizável. Inicialmente, trabalharemos com os diagramas obtidos a partir da corrente de tetraquark (3.1):

$$
j_{\mu}=\frac{\epsilon_{a b c} \epsilon_{d e c}}{\sqrt{2}}\left[\left(q_{a}^{T} C \gamma_{5} c_{b}\right)\left(\bar{q}_{d} \gamma_{\mu} \gamma_{5} C \bar{c}_{e}^{T}\right)+\left(q_{a}^{T} C \gamma_{5} \gamma_{\mu} c_{b}\right)\left(\bar{q}_{d} \gamma_{5} C \bar{c}_{e}^{T}\right)\right]
$$

quando $q=s$ obtemos o tetraquark $[c s \bar{c} \bar{s}]$ e quando $q=u, d$ obtemos o tetraquark $[c q \bar{c} \bar{q}]$.

Vale lembrar que cada diagrama é calculado a partir de (3.15):

$$
\left.\begin{array}{rl}
\Pi\left(q^{2}\right)= & -\frac{i \epsilon_{a b c} \epsilon_{d e c} \epsilon_{i j k} \epsilon_{l m k}}{6 q^{2}} \int \frac{d^{4} x d^{4} p_{1} d^{4} p_{2}}{(2 \pi)^{8}} e^{i q \cdot x} e^{-i p_{1} \cdot x} e^{-i p_{2} \cdot x} \\
& \times\left\{\begin{array}{ccc}
\operatorname{Tr}\left[S_{b i}^{c}\left(p_{1}\right) \gamma_{5} C S_{a j}^{q^{T}}(x) C \gamma_{5}\right] \cdot \operatorname{Tr}\left[S_{m d}^{q}(-x) \gamma_{\mu} \gamma_{5} C S_{l e}^{c^{T}}\left(-p_{2}\right) C \gamma_{5} \gamma^{\mu}\right] & + \\
+ & \operatorname{Tr}\left[S_{b i}^{c}\left(p_{1}\right) \gamma^{\mu} \gamma_{5} C S_{a j}^{q^{T}}(x) C \gamma_{5}\right] \cdot \operatorname{Tr}\left[S_{m d}^{q}(-x) \gamma_{\mu} \gamma_{5} C S_{l e}^{T^{T}}\left(-p_{2}\right) C \gamma_{5}\right] & + \\
+ & \operatorname{Tr}\left[S_{b i}^{c}\left(p_{1}\right) \gamma_{5} C S_{a j}^{q^{T}}(x) C \gamma_{5} \gamma^{\mu}\right] \cdot \operatorname{Tr}\left[S_{m d}^{q}(-x) \gamma_{5} C S_{l e}^{c^{T}}\left(-p_{2}\right) C \gamma_{5} \gamma_{\mu}\right] & + \\
+ & \operatorname{Tr}\left[S_{b i}^{c}\left(p_{1}\right) \gamma^{\mu} \gamma_{5} C S_{a j}^{q^{T}}(x) C \gamma_{5} \gamma_{\mu}\right] \cdot \operatorname{Tr}\left[S_{m d}^{q}(-x) \gamma_{5} C S_{l e}^{c^{T}}\left(-p_{2}\right) C \gamma_{5}\right] .
\end{array}\right.
\end{array}\right\}
$$

\section{C.1 Contribuição Perturbativa}

Como exemplo, calculemos as contribuições perturbativas da função de correlação dadas pelos diagramas:

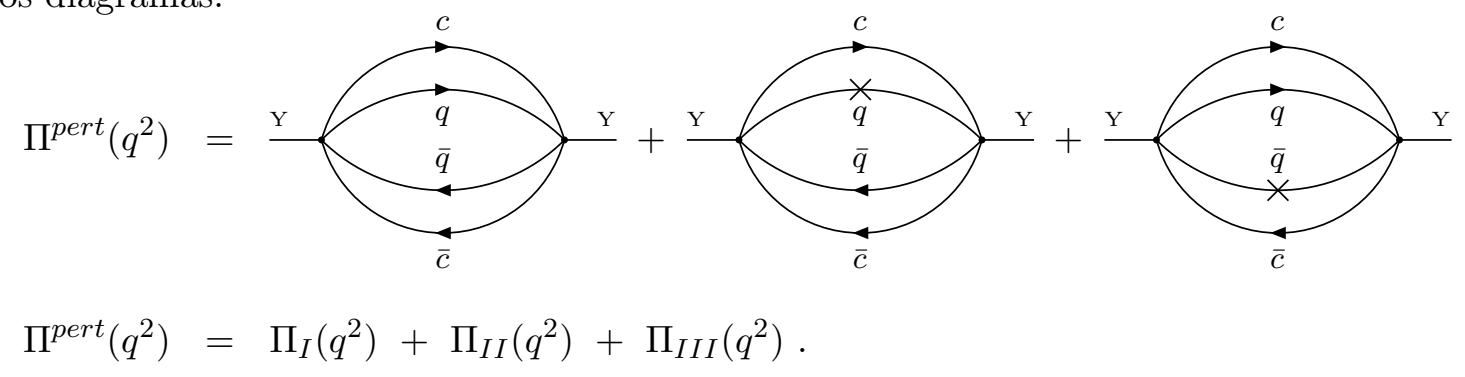


Para o primeiro diagrama, há apenas os propagadores de quarks livres (A.3) e (A.12):

$$
\begin{aligned}
& \text { Quarks: } \quad S_{b i}^{c}(p)=\frac{i\left(\not p+m_{c}\right) \delta_{b i}}{p^{2}-m_{c}^{2}} \quad S_{a j}^{q}(x)=\frac{i \not \delta_{a j}}{2 \pi^{2} x^{4}} . \\
& \text { Antiquarks: } S_{m d}^{q}(-x)=-\frac{i \not \not \delta_{m d}}{2 \pi^{2} x^{4}} \quad S_{l e}^{c}(-p)=-\frac{i\left(\not p-m_{c}\right) \delta_{l e}}{p^{2}-m_{c}^{2}} \text {. }
\end{aligned}
$$

Inserindo-os em (C.1), obtemos:

$$
\begin{aligned}
& \Pi_{I}\left(q^{2}\right)=-\frac{i}{3 \cdot 2^{9} \pi^{12} q^{2}} \int \frac{d^{4} x d^{4} p_{1} d^{4} p_{2}}{x^{8}\left(p_{1}^{2}-m_{c}^{2}\right)\left(p_{2}^{2}-m_{c}^{2}\right)} e^{i q \cdot x} e^{-i p_{1} \cdot x} e^{-i p_{2} \cdot x}
\end{aligned}
$$

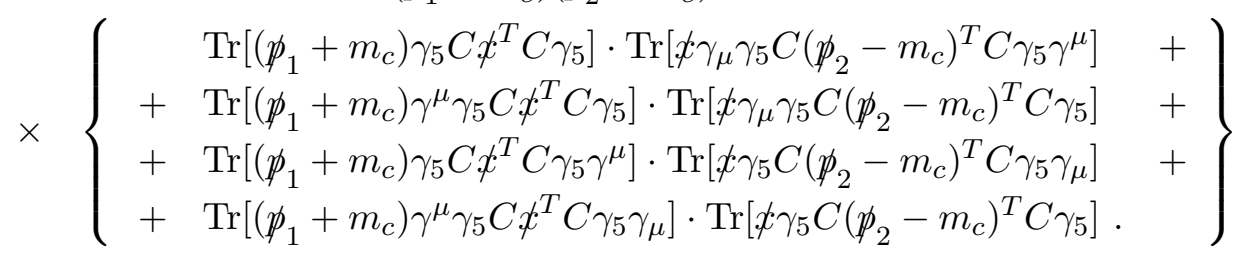

Simplificando e calculando os traços:

$$
\Pi_{I}\left(q^{2}\right)=\frac{i}{2^{4} \pi^{12} q^{2}} \int \frac{d^{4} x d^{4} p_{1} d^{4} p_{2}}{x^{8}\left(p_{1}^{2}-m_{c}^{2}\right)\left(p_{2}^{2}-m_{c}^{2}\right)} e^{i q \cdot x} e^{-i p_{1} \cdot x} e^{-i p_{2} \cdot x}\left[2\left(x \cdot p_{1}\right)\left(x \cdot p_{2}\right)+m_{c}^{2} x^{2}\right] .
$$

De forma análoga, para os demais diagramas basta inserirmos o propagador (A.4):

$$
S_{a b}^{q}(x)=-\frac{m_{q} \delta_{a b}}{4 \pi^{2} x^{2}}
$$

para obtermos a correção, em primeira ordem, na massa do quark leve $q$. As expressões para $\Pi_{I I}\left(q^{2}\right)$ e $\Pi_{I I I}\left(q^{2}\right)$ são dadas respectivamente por:

$$
\begin{aligned}
\Pi_{I I}\left(q^{2}\right) & =-\frac{m_{q} m_{c}}{2^{5} \pi^{12} q^{2}} \int \frac{d^{4} x d^{4} p_{1} d^{4} p_{2}}{x^{6}\left(p_{1}^{2}-m_{c}^{2}\right)\left(p_{2}^{2}-m_{c}^{2}\right)} e^{i q \cdot x} e^{-i p_{1} \cdot x} e^{-i p_{2} \cdot x}\left[\left(x \cdot p_{1}\right)-\left(x \cdot p_{2}\right)\right] \\
\Pi_{I I I}\left(q^{2}\right) & =\frac{m_{q} m_{c}}{2^{5} \pi^{12} q^{2}} \int \frac{d^{4} x d^{4} p_{1} d^{4} p_{2}}{x^{6}\left(p_{1}^{2}-m_{c}^{2}\right)\left(p_{2}^{2}-m_{c}^{2}\right)} e^{i q \cdot x} e^{-i p_{1} \cdot x} e^{-i p_{2} \cdot x}\left[\left(x \cdot p_{1}\right)-\left(x \cdot p_{2}\right)\right] .
\end{aligned}
$$

Como essas integrais são simétricas pela troca $p_{1} \leftrightarrow p_{2}$, então

$$
\Pi_{I I}\left(q^{2}\right)=\Pi_{I I I}\left(q^{2}\right)=0
$$

Logo, os termos de correção na massa não contribuem para a função de correlação perturbativa, que tem seu valor dado pelo termo dominante $\Pi_{I}\left(q^{2}\right)$. Desta forma, utilizando as relações contidas no Apêndice B, obtemos:

$$
\Pi^{p e r t}\left(q^{2}\right)=\frac{i}{2^{4} \pi^{12} q^{2}} \int \frac{d^{4} x d^{4} p_{1} d^{4} p_{2}}{x^{8}\left(p_{1}^{2}-m_{c}^{2}\right)\left(p_{2}^{2}-m_{c}^{2}\right)} e^{i q \cdot x} e^{-i p_{1} \cdot x} e^{-i p_{2} \cdot x}\left[2\left(x \cdot p_{1}\right)\left(x \cdot p_{2}\right)+m_{c}^{2} x^{2}\right]
$$




$$
\begin{aligned}
\Pi^{p e r t}\left(q^{2}\right) & \stackrel{(B .41)}{=} \frac{i}{2^{5} \pi^{8} q^{2}} \int_{0}^{\infty} \frac{d \alpha d \beta}{\alpha^{3} \beta^{3}} e^{-m_{c}^{2}(\alpha+\beta)} \int \frac{d^{4} x}{x^{4}} e^{i q \cdot x+(\alpha+\beta) \frac{x^{2}}{4 \alpha \beta}}\left(1-\frac{2 \alpha \beta m_{c}^{2}}{x^{2}}\right) \\
& \stackrel{(B .45)}{=} \frac{1}{2^{7} \pi^{6} q^{2}} \int_{0}^{\infty} \frac{d \alpha d \beta d \gamma}{\alpha \beta \gamma^{2}} \frac{e^{-m_{c}^{2}(\alpha+\beta)+\frac{\alpha \beta \gamma q^{2}}{\alpha \beta+\beta \gamma+\gamma \alpha}}}{(\alpha \beta+\beta \gamma+\gamma \alpha)^{2}}\left(4 \gamma+\alpha \beta m_{c}^{2}\right) \\
& \stackrel{(B .47)}{=} \frac{1}{2^{7} \pi^{6} q^{2}} \int_{0}^{1} \frac{d \alpha d \beta}{\alpha^{3} \beta^{3}} \int_{0}^{\infty} \frac{d \lambda}{\lambda^{5}} \theta(1-\alpha-\beta)(1-\alpha-\beta)\left[4+m_{c}^{2} \lambda(1-\alpha-\beta)\right] e^{-\lambda E(\alpha, \beta)} \\
& \stackrel{(B .6 .6)}{=} \frac{1}{3 \cdot 2^{8} \pi^{6} q^{2}} \int_{0}^{1} \frac{d \alpha d \beta}{\alpha^{3} \beta^{3}} \theta(1-\alpha-\beta)(1-\alpha-\beta) E^{3}(\alpha, \beta)\left[m_{c}^{2}(1-\alpha-\beta)-E(\alpha, \beta)\right] \ln E(\alpha, \beta)
\end{aligned}
$$

onde $E(\alpha, \beta)=m_{c}^{2}(\alpha+\beta)-\alpha \beta q^{2}$. Como já foi observado, a expressão de $\Pi^{\text {pert }}\left(q^{2}\right)$ é convenientemente descrita em termos de uma relação de dispersão, que nos permite determinar a densidade espectral através de $\left(s=q^{2}\right)$ :

$$
\rho^{\text {pert }}(s)=\frac{1}{\pi} \operatorname{Im}\left[\Pi^{\text {pert }}(s)\right] .
$$

Analisando (C.4), sua parte imaginária existe somente se o termo contido no logaritmo satisfizer a condição $E(\alpha, \beta)<0$ que em termos da variável $s$ fica:

$$
m_{c}^{2}(\alpha+\beta)-\alpha \beta s<0
$$

Além disso, a função de Heaviside $\theta(1-\alpha-\beta)$ nos fornece uma outra condição:

$$
\alpha+\beta \leq 1 .
$$

Juntas, (C.6) e (C.7) impõe os seguintes limites para os valores de $\alpha, \beta$ e $s$ :

$$
\begin{gathered}
\frac{1-\sqrt{1-4 m_{c}^{2} / s}}{2}<\alpha<\frac{1+\sqrt{1-4 m_{c}^{2} / s}}{2} \\
\frac{\alpha m_{c}^{2}}{\left(s \alpha-m_{c}^{2}\right)}<\beta<1-\alpha .
\end{gathered}
$$

Como a variável $\alpha$ é real, então o termo dentro da raiz quadrada em (C.8) deve ser positivo. Donde concluímos que:

$$
s>4 m_{c}^{2} .
$$

Portanto, considerando a relação (B.35) a densidade espectral $\rho^{\text {pert }}(s)$ fica dada por:

$$
\rho^{\text {pert }}(s)=-\frac{1}{3 \cdot 2^{8} \pi^{6} s} \int_{\alpha_{\min }}^{\alpha_{\max }} \frac{d \alpha}{\alpha^{3}} \int_{\beta_{\min }}^{1-\alpha} \frac{d \beta}{\beta^{3}}(1-\alpha-\beta)\left[m_{c}^{2}(\alpha+\beta)-\alpha \beta s\right]^{3}\left[m_{c}^{2}(1-2 \alpha-2 \beta)+\alpha \beta s\right]
$$

onde $\alpha_{\min }, \alpha_{\max }$ e $\beta_{\text {min }}$ são os limites mostrados em (C.8) e (C.9). 


\section{C.2 Contribuição não-Perturbativa}

Para as contribuições não-perturbativas, tomemos como exemplo o cálculo da função de correlação (C.1) dos condensados de glúons. Para esta contribuição admitimos os seguintes diagramas:

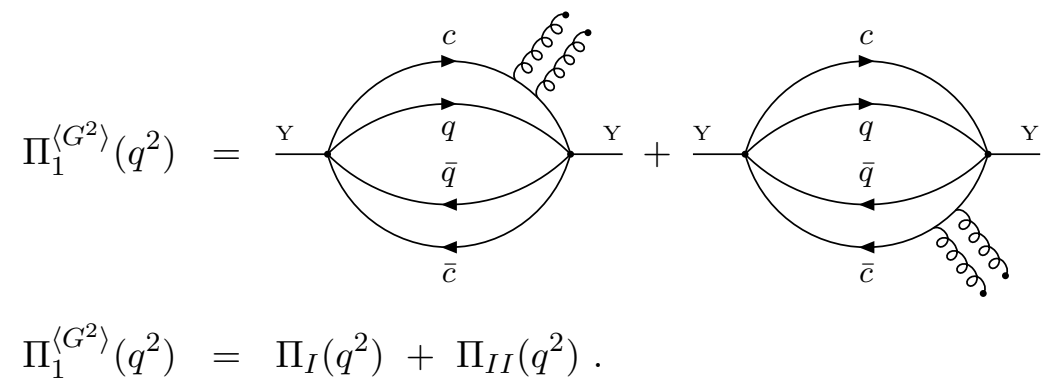

Para o primeiro diagrama, temos que utilizar os propagadores (A.3), (A.12) e (A.14) na linha dos quarks e antiquarks da seguinte forma:

$$
\begin{aligned}
& \text { Quarks : } \quad S_{b i}^{c}(p)=\frac{i \delta_{b i}}{12}\left[\frac{p^{2}+m_{c} \not p}{\left(p^{2}-m_{c}^{2}\right)^{4}}\right] \quad S_{a j}^{q}(x)=\frac{i \not \delta_{a j}}{2 \pi^{2} x^{4}} . \\
& \text { Antiquarks : } S_{m d}^{q}(-x)=-\frac{i \not \not \delta_{m d}}{2 \pi^{2} x^{4}} \quad S_{l e}^{c}(-p)=\frac{i\left(-\not p+m_{c}\right) \delta_{l e}}{p^{2}-m_{c}^{2}} \text {. }
\end{aligned}
$$

Inserindo-os em (C.1), obtemos:

$$
\begin{aligned}
& \Pi_{I}\left(q^{2}\right)=-\frac{i m_{c}\left\langle g_{s}^{2} G^{2}\right\rangle}{3 \cdot 2^{11} \pi^{12} q^{2}} \int \frac{d^{4} x d^{4} p_{1} d^{4} p_{2}}{x^{8}\left(p_{1}^{2}-m_{c}^{2}\right)^{4}\left(p_{2}^{2}-m_{c}^{2}\right)} e^{i q \cdot x} e^{-i p_{1} \cdot x} e^{-i p_{2} \cdot x}
\end{aligned}
$$

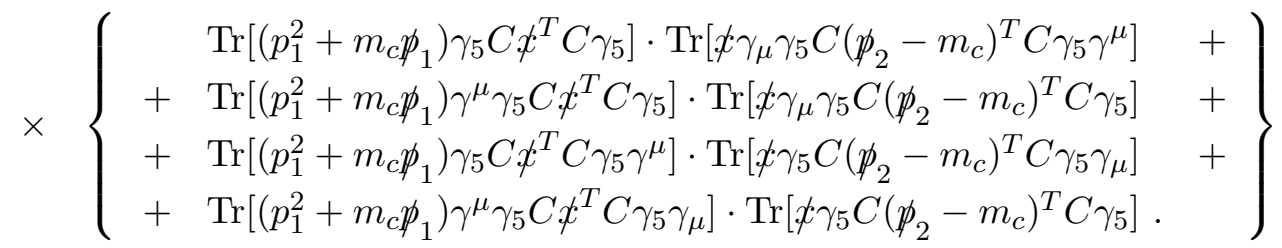

Simplificando e calculando os traços:

$\Pi_{I}\left(q^{2}\right)=\frac{i m_{c}^{2}\left\langle g_{s}^{2} G^{2}\right\rangle}{3 \cdot 2^{6} \pi^{12} q^{2}} \int \frac{d^{4} x d^{4} p_{1} d^{4} p_{2}}{x^{8}\left(p_{1}^{2}-m_{c}^{2}\right)^{4}\left(p_{2}^{2}-m_{c}^{2}\right)} e^{i q \cdot x} e^{-i p_{1} \cdot x} e^{-i p_{2} \cdot x}\left[2\left(x \cdot p_{1}\right)\left(x \cdot p_{2}\right)+x^{2} p_{1}^{2}\right]$.

De forma análoga, para o segundo diagrama basta inserirmos em (C.1), o propagador (A.14) na linha do antiquark pesado $c$. A expressão obtida para $\Pi_{I I}\left(q^{2}\right)$ é dada por:

$\Pi_{I I}\left(q^{2}\right)=\frac{i m_{c}^{2}\left\langle g_{s}^{2} G^{2}\right\rangle}{3 \cdot 2^{6} \pi^{12} q^{2}} \int \frac{d^{4} x d^{4} p_{1} d^{4} p_{2}}{x^{8}\left(p_{1}^{2}-m_{c}^{2}\right)^{4}\left(p_{2}^{2}-m_{c}^{2}\right)} e^{i q \cdot x} e^{-i p_{1} \cdot x} e^{-i p_{2} \cdot x}\left[2\left(x \cdot p_{1}\right)\left(x \cdot p_{2}\right)+x^{2} p_{1}^{2}\right]$ donde vemos que a contribuição dos dois diagramas são iguais:

$$
\Pi_{I}\left(q^{2}\right)=\Pi_{I I}\left(q^{2}\right)
$$


Logo, a função de correlação dos condensados de glúons para os tetraquarks é dada por:

$$
\begin{aligned}
\Pi_{1}^{\left\langle G^{2}\right\rangle}\left(q^{2}\right) & =2 \Pi_{I}\left(q^{2}\right) \\
\Pi_{1}^{\left\langle G^{2}\right\rangle}\left(q^{2}\right) & =\frac{i m_{c}^{2}\left\langle g_{s}^{2} G^{2}\right\rangle}{3 \cdot 2^{5} \pi^{12} q^{2}} \int \frac{d^{4} x d^{4} p_{1} d^{4} p_{2}}{x^{8}\left(p_{1}^{2}-m_{c}^{2}\right)^{4}\left(p_{2}^{2}-m_{c}^{2}\right)} e^{i q \cdot x} e^{-i p_{1} \cdot x} e^{-i p_{2} \cdot x}\left[2\left(x \cdot p_{1}\right)\left(x \cdot p_{2}\right)+x^{2} p_{1}^{2}\right]
\end{aligned}
$$

convenientemente, podemos reescrêve-la como:

$$
\Pi_{1}^{\left\langle G^{2}\right\rangle}\left(q^{2}\right)=\frac{i m_{c}^{2}\left\langle g_{s}^{2} G^{2}\right\rangle}{3 \cdot 2^{5} \pi^{12} q^{2}} \int \frac{d^{4} x d^{4} p_{1} d^{4} p_{2}}{x^{8}\left(p_{1}^{2}-m_{c}^{2}\right)^{3}\left(p_{2}^{2}-m_{c}^{2}\right)} e^{i q \cdot x} e^{-i p_{1} \cdot x} e^{-i p_{2} \cdot x}\left[\frac{2\left(x \cdot p_{1}\right)\left(x \cdot p_{2}\right)}{p_{1}^{2}-m_{c}^{2}}+x^{2}\left(1+\frac{m_{c}^{2}}{p_{1}^{2}-m_{c}^{2}}\right)\right] .
$$

Então, utilizando as relações contidas no Apêndice B, calculamos $\Pi_{1}^{\left\langle G^{2}\right\rangle}\left(q^{2}\right)$ :

$$
\begin{aligned}
& \Pi_{1}^{\left\langle G^{2}\right\rangle}\left(q^{2}\right) \stackrel{(B .41)}{=}-\frac{i m_{c}^{2}\left\langle g_{s}^{2} G^{2}\right\rangle}{3^{2} \cdot 2^{6} \pi^{8} q^{2}} \int \frac{d \alpha d \beta}{\beta^{2}} e^{-m_{c}^{2}(\alpha+\beta)} \int \frac{d^{4} x}{x^{6}} e^{i q \cdot x+(\alpha+\beta) \frac{x^{2}}{4 \alpha \beta}}\left[\frac{x^{2}}{2 \beta}+3-\alpha m_{c}^{2}\right] \\
& \stackrel{(B .45)}{=} \quad-\frac{m_{c}^{2}\left\langle g_{s}^{2} G^{2}\right\rangle}{3^{2} \cdot 2^{7} \pi^{6} q^{2}} \int_{0}^{\infty} \frac{d \alpha d \beta d \gamma}{\alpha^{-2} \gamma} \frac{e^{-m_{c}^{2}(\alpha+\beta)+\frac{\alpha \beta \gamma q^{2}}{\alpha \beta+\beta \gamma+\gamma \alpha}}}{(\alpha \beta+\beta \gamma+\gamma \alpha)^{2}}\left[\frac{1}{\beta}-\frac{3}{4 \gamma}+\frac{m_{c}^{2} \alpha}{4 \gamma}\right] \\
& \stackrel{(B .47)}{=}-\frac{m_{c}^{2}\left\langle g_{s}^{2} G^{2}\right\rangle}{3^{2} \cdot 2^{7} \pi^{6} q^{2}} \int_{0}^{1} \frac{d \alpha d \beta}{\beta^{3}} \theta(1-\alpha-\beta)(1-\alpha-\beta) \int_{0}^{\infty} \frac{d \lambda}{\lambda}\left[\frac{1}{\lambda}-\frac{3(1-\alpha-\beta)}{4 \alpha \lambda}+\frac{m_{c}^{2}(1-\alpha-\beta)}{4}\right] e^{-\lambda E(\alpha, \beta)} \\
& \stackrel{(B .6 .6)}{=}-\frac{m_{c}^{2}\left\langle g_{s}^{2} G^{2}\right\rangle}{3^{2} \cdot 2^{9} \pi^{6} q^{2}} \int_{0}^{1} \frac{d \alpha d \beta}{\alpha \beta^{3}} \theta(1-\alpha-\beta)(1-\alpha-\beta)\left\{(7 \alpha+3 \beta-3) E(\alpha, \beta)-m_{c}^{2} \alpha(1-\alpha-\beta)\right\} \ln E(\alpha, \beta)
\end{aligned}
$$

onde $E(\alpha, \beta)=m_{c}^{2}(\alpha+\beta)-\alpha \beta q^{2}$. Como já foi observado, a expressão de $\Pi_{1}^{\left\langle G^{2}\right\rangle}\left(q^{2}\right)$ é convenientemente descrita em termos de uma relação de dispersão, o que nos permite determinar a densidade espectral através de $\left(s=q^{2}\right)$ :

$$
\rho_{1}^{\left\langle G^{2}\right\rangle}(s)=\frac{1}{\pi} \operatorname{Im}\left[\Pi_{1}^{\left\langle G^{2}\right\rangle}(s)\right]
$$

Como no caso das contribuições perturbativas, (C.14) possuirá uma parte imaginária somente se o termo contido no logaritmo satisfizer:

$$
m_{c}^{2}(\alpha+\beta)-\alpha \beta s<0
$$

Logo, obtemos os mesmos valores limites $\alpha_{\min }, \alpha_{\max }$ e $\beta_{\min }$ mostrados em (C.8) e (C.9). Portanto, considerando a relação (B.35) a densidade espectral $\rho_{1}^{\left\langle G^{2}\right\rangle}(s)$ fica dada por:

$$
\rho_{1}^{\left\langle G^{2}\right\rangle}(s)=\frac{m_{c}^{2}\left\langle g_{s}^{2} G^{2}\right\rangle}{3^{2} \cdot 2^{9} \pi^{6} s} \int_{\alpha_{\min }}^{\alpha_{\max }} \frac{d \alpha}{\alpha} \int_{\beta_{\min }}^{1-\alpha} \frac{d \beta}{\beta^{3}}(1-\alpha-\beta)\left\{(7 \alpha+3 \beta-3)\left[m_{c}^{2}(\alpha+\beta)-\alpha \beta s\right]-m_{c}^{2} \alpha(1-\alpha-\beta)\right\} .
$$




\section{C.3 Contribuição não-Fatorizável}

Há também a contribuição de termos não-fatorizáveis para os condensados de glúons. Para determiná-los, admitimos os seguintes diagramas:

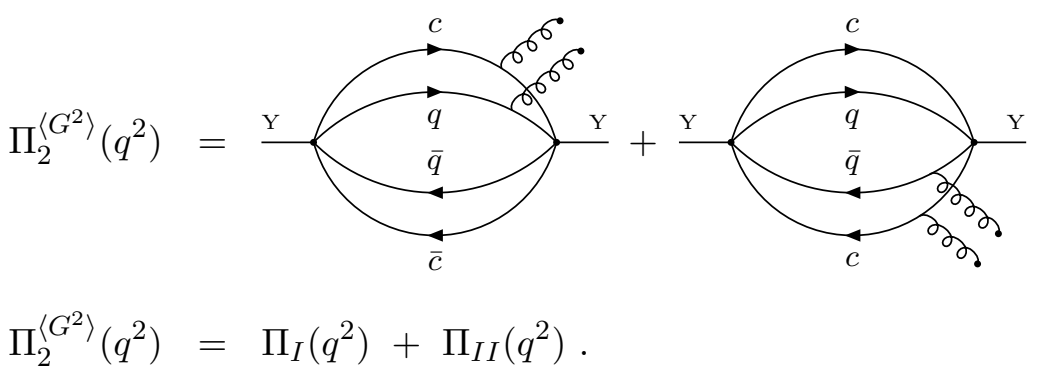

Para o primeiro diagrama, temos que utilizar os propagadores (A.3), (A.9), (A.12) e (A.13) na linha dos quarks e antiquarks da seguinte forma:

$$
\begin{aligned}
& \text { Quarks: } \quad S_{b i}^{c}(p)=-\frac{i t_{b i}^{n}}{4}\left[\frac{\not p \sigma^{\mu \nu}+\sigma^{\mu \nu} \not p+2 m_{c} \sigma^{\mu \nu}}{\left(p^{2}-m_{c}^{2}\right)^{2}}\right] \quad S_{a j}^{q}(x)=-\frac{i\left(\not \not \sigma_{\mu \nu}+\sigma_{\mu \nu} \not\right) t_{a j}^{n}}{3 \cdot 2^{9} \pi^{2} x^{2}}\left\langle g_{s}^{2} G^{2}\right\rangle \text {. } \\
& \text { Antiquarks: } S_{m d}^{q}(-x)=-\frac{i \not \not \delta_{m d}}{2 \pi^{2} x^{4}} \quad S_{l e}^{c}(-p)=\frac{i\left(-\not p+m_{c}\right) \delta_{l e}}{p^{2}-m_{c}^{2}} .
\end{aligned}
$$

Inserindo-os em (C.1), obtemos:

$$
\begin{aligned}
& \Pi_{I}\left(q^{2}\right)=\frac{i\left\langle g_{s}^{2} G^{2}\right\rangle}{3^{2} \cdot 2^{18} \pi^{12} q^{2}} \int \frac{d^{4} x d^{4} p_{1} d^{4} p_{2}}{x^{6}\left(p_{1}^{2}-m_{c}^{2}\right)^{2}\left(p_{2}^{2}-m_{c}^{2}\right)} e^{i q \cdot x} e^{-i p_{1} \cdot x} e^{-i p_{2} \cdot x}
\end{aligned}
$$

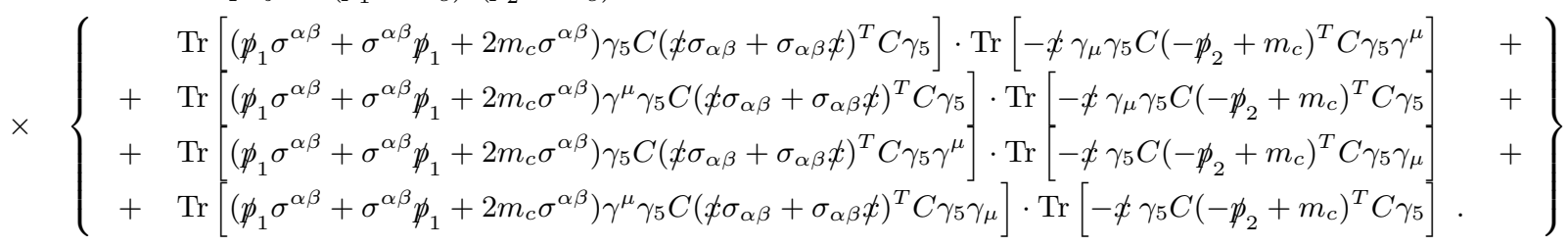

Simplificando e calculando os traços:

$$
\Pi_{I}\left(q^{2}\right)=\frac{i m_{c}^{2}\left\langle g_{s}^{2} G^{2}\right\rangle}{3 \cdot 2^{10} \pi^{12} q^{2}} \int \frac{d^{4} x d^{4} p_{1} d^{4} p_{2}}{x^{4}\left(p_{1}^{2}-m_{c}^{2}\right)\left(p_{2}^{2}-m_{c}^{2}\right)} e^{i q \cdot x} e^{-i p_{1} \cdot x} e^{-i p_{2} \cdot x} .
$$

De forma análoga, para o segundo diagrama a expressão para $\Pi_{I I}\left(q^{2}\right)$ é dada por:

$$
\Pi_{I I}\left(q^{2}\right)=\frac{i m_{c}^{2}\left\langle g_{s}^{2} G^{2}\right\rangle}{3 \cdot 2^{10} \pi^{12} q^{2}} \int \frac{d^{4} x d^{4} p_{1} d^{4} p_{2}}{x^{4}\left(p_{1}^{2}-m_{c}^{2}\right)\left(p_{2}^{2}-m_{c}^{2}\right)} e^{i q \cdot x} e^{-i p_{1} \cdot x} e^{-i p_{2} \cdot x}
$$

donde vemos que a contribuição dos dois diagramas são iguais:

$$
\Pi_{I}\left(q^{2}\right)=\Pi_{I I}\left(q^{2}\right)
$$

e que nos fornece:

$$
\Pi_{2}^{\left\langle G^{2}\right\rangle}\left(q^{2}\right)=\frac{i m_{c}^{2}\left\langle g_{s}^{2} G^{2}\right\rangle}{3 \cdot 2^{9} \pi^{12} q^{2}} \int \frac{d^{4} x d^{4} p_{1} d^{4} p_{2}}{x^{4}\left(p_{1}^{2}-m_{c}^{2}\right)\left(p_{2}^{2}-m_{c}^{2}\right)} e^{i q \cdot x} e^{-i p_{1} \cdot x} e^{-i p_{2} \cdot x} .
$$


Logo, utilizando as relações contidas no Apêndice B, calculamos o termo não-fatorizável da função de correlação dos condensados de glúons:

$$
\begin{aligned}
\Pi_{2}^{\left\langle G^{2}\right\rangle}\left(q^{2}\right) & \stackrel{(B .41)}{=} \frac{i m_{c}^{2}\left\langle g_{s}^{2} G^{2}\right\rangle}{3 \cdot 2^{9} \pi^{8} q^{2}} \int_{0}^{\infty} \frac{d \alpha d \beta}{\alpha \beta^{2}} e^{-m_{c}^{2}(\alpha+\beta)} \int \frac{d^{4} x}{x^{4}} e^{i q \cdot x+(\alpha+\beta) \frac{x^{2}}{4 \alpha \beta}} \\
& \stackrel{(B .45)}{=} \frac{m_{c}^{2}\left\langle g_{s}^{2} G^{2}\right\rangle}{3 \cdot 2^{9} \pi^{6} q^{2}} \int_{0}^{\infty} \frac{d \alpha d \beta d \gamma}{\alpha^{-1} \gamma} \frac{e^{-m_{c}^{2}(\alpha+\beta)+\frac{\alpha \beta \gamma q^{2}}{\alpha \beta+\beta \gamma+\gamma \alpha}}}{(\alpha \beta+\beta \gamma+\gamma \alpha)^{2}} \\
& \stackrel{(B .47)}{=} \frac{m_{c}^{2}\left\langle g_{s}^{2} G^{2}\right\rangle}{3 \cdot 2^{9} \pi^{6} q^{2}} \int_{0}^{1} \frac{d \alpha d \beta}{\alpha \beta^{2}} \theta(1-\alpha-\beta)(1-\alpha-\beta) \int_{0}^{\infty} \frac{d \lambda}{\lambda^{2}} e^{-\lambda E(\alpha, \beta)} \\
& \stackrel{(B .6 .6)}{=} \frac{m_{c}^{2}\left\langle g_{s}^{2} G^{2}\right\rangle}{3 \cdot 2^{9} \pi^{6} q^{2}} \int_{0}^{1} \frac{d \alpha d \beta}{\alpha \beta^{2}} \theta(1-\alpha-\beta)(1-\alpha-\beta) E(\alpha, \beta) \ln E(\alpha, \beta)
\end{aligned}
$$

onde $E(\alpha, \beta)=m_{c}^{2}(\alpha+\beta)-\alpha \beta q^{2}$. Temos que a densidade espectral é obtida por $\left(s=q^{2}\right)$ :

$$
\rho_{2}^{\left\langle G^{2}\right\rangle}(s)=\frac{1}{\pi} \operatorname{Im}\left[\Pi_{1}^{\left\langle G^{2}\right\rangle}(s)\right] .
$$

Então, como nos casos anteriores, (C.20) possuirá uma parte imaginária somente se o termo contido no logaritmo satisfizer:

$$
m_{c}^{2}(\alpha+\beta)-\alpha \beta s<0
$$

que determina os valores limites (C.8) e (C.9). Então, considerando a relação (B.35), a densidade espectral $\rho_{2}^{\left\langle G^{2}\right\rangle}(s)$ fica dada por:

$$
\rho_{2}^{\left\langle G^{2}\right\rangle}(s)=-\frac{m_{c}^{2}\left\langle g_{s}^{2} G^{2}\right\rangle}{3 \cdot 2^{9} \pi^{6} s} \int_{\alpha_{\min }}^{\alpha_{\max }} \frac{d \alpha}{\alpha} \int_{\beta_{\min }}^{1-\alpha} \frac{d \beta}{\beta^{2}}(1-\alpha-\beta)\left[m_{c}^{2}(\alpha+\beta)-\alpha \beta s\right] .
$$

Por fim, somando as contribuições (C.17) e (C.23), obtemos a contribuição total dos condensados de glúons para a densidade espectral da OPE:

$$
\begin{aligned}
& \rho^{\left\langle G^{2}\right\rangle}(s)=\rho_{1}^{\left\langle G^{2}\right\rangle}(s)+\rho_{2}^{\left\langle G^{2}\right\rangle}(s) \\
& \rho^{\left\langle G^{2}\right\rangle}(s)=\frac{m_{c}^{2}\left\langle g_{s}^{2} G^{2}\right\rangle}{3^{2} \cdot 2^{9} \pi^{6} s} \int_{\alpha_{\min }}^{\alpha_{\max }} \frac{d \alpha}{\alpha} \int_{\beta_{\min }}^{1-\alpha} \frac{d \beta}{\beta^{3}}(1-\alpha-\beta)\left\{(7 \alpha-3)\left[m_{c}^{2}(\alpha+\beta)-\alpha \beta s\right]-m_{c}^{2} \alpha(1-\alpha-\beta)\right\}
\end{aligned}
$$

que é a densidade espectral (3.19) mostrada no Capítulo 3. 



\section{Apêndice D}

\section{Cálculo dos Diagramas Moleculares}

No caso das moléculas, temos algumas diferenças no cálculo dos traços contidos na função de correlação. Tomando os mesmos diagramas calculados para os tetraquarks (perturbativo, condensado de quarks e condesado de glúons) veremos ao final a relação que há entre estas duas descrições. Trabalharemos com os diagramas obtidos a partir da corrente molecular (3.40):

$$
\tilde{j}_{\mu}=\frac{1}{\sqrt{2}}\left[\left(\bar{q} \gamma_{\mu} c\right)(\bar{c} q)+\left(\bar{c} \gamma_{\mu} q\right)(\bar{q} c)\right]
$$

quando $q=s$ obtemos a molécula $D_{s 0} \bar{D}_{s}^{*}$ e quando $q=u, d$ obtemos a molécula $D_{0} \bar{D}^{*}$. A segunda opção será escolhida para exemplificarmos as contas das seções seguintes.

Vale lembrar que cada diagrama é calculado a partir de (3.45):

$$
\begin{aligned}
& \tilde{\Pi}\left(q^{2}\right)=-\frac{i}{6 q^{2}} \int \frac{d^{4} x d^{4} p_{1} d^{4} p_{2}}{(2 \pi)^{8}} e^{i q \cdot x} e^{-i p_{1} \cdot x} e^{-i p_{2} \cdot x} \\
& \times\left\{\begin{array}{rcc}
\operatorname{Tr}\left[\gamma_{\mu} S_{a i}^{c}\left(p_{1}\right) S_{i a}^{q}(-x)\right] \cdot \operatorname{Tr}\left[S_{b j}^{q}(x) \gamma^{\mu} S_{j b}^{c}\left(-p_{2}\right)\right] & + \\
+\operatorname{Tr}\left[S_{a i}^{c}\left(p_{1}\right) \gamma^{\mu} S_{i a}^{q}(-x)\right] \cdot \operatorname{Tr}\left[\gamma_{\mu} S_{b j}^{q}(x) S_{j b}^{c}\left(-p_{2}\right)\right] & + \\
+\operatorname{Tr}\left[\gamma_{\mu} S_{a i}^{c}\left(p_{1}\right) \gamma^{\mu} S_{i a}^{q}(-x)\right] \cdot \operatorname{Tr}\left[S_{b j}^{q}(x) S_{j b}^{c}\left(-p_{2}\right)\right] & + \\
+\operatorname{Tr}\left[S_{a i}^{c}\left(p_{1}\right) S_{i a}^{q}(-x)\right] \cdot \operatorname{Tr}\left[\gamma_{\mu} S_{b j}^{q}(x) \gamma^{\mu} S_{j b}^{c}\left(-p_{2}\right)\right] . &
\end{array}\right\}
\end{aligned}
$$

\section{D.1 Contribuição Perturbativa}

Os diagramas perturbativos considerados para a função de correlação são dados por:

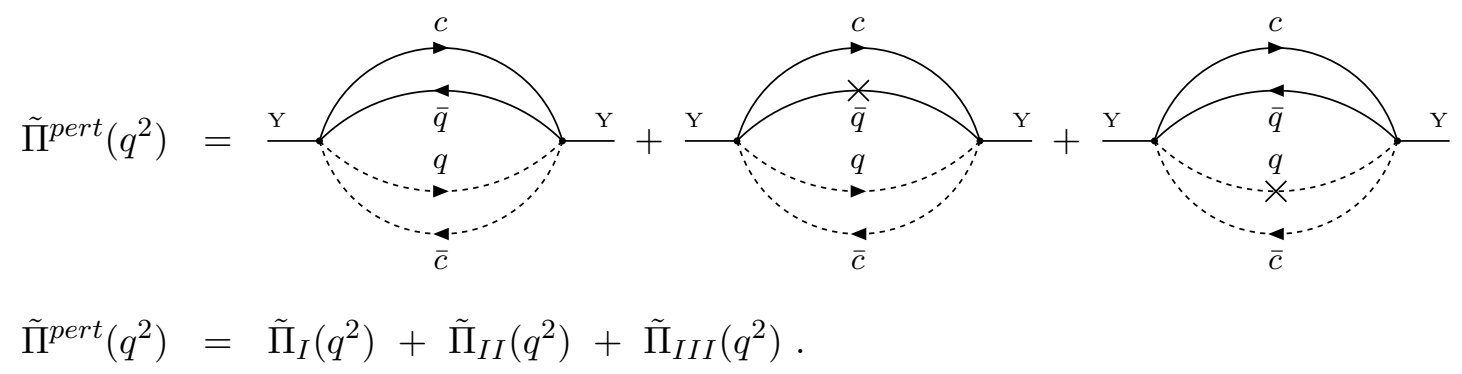


Comecemos então pelo primeiro diagrama que apresenta apenas os propagadores de quarks livres (A.3) e (A.12):

$$
\begin{array}{llrl}
\text { Méson } D_{0}: & S_{a i}^{c}\left(p_{1}\right)=\frac{i\left(\not p_{1}+m_{c}\right) \delta_{a i}}{p_{1}^{2}-m_{c}^{2}} & S_{i a}^{q}(-x) & =-\frac{i \not \not \delta_{i a}}{2 \pi^{2} x^{4}} . \\
\text { Méson } \bar{D}^{*}: & S_{b j}^{q}(x)=\frac{i \not \not \delta_{b j}}{2 \pi^{2} x^{4}} & S_{j b}^{c}\left(-p_{2}\right) & =-\frac{i\left(\not p_{2}-m_{c}\right) \delta_{j b}}{p_{2}^{2}-m_{c}^{2}} .
\end{array}
$$

Inserindo-os em (D.1), obtemos:

$$
\begin{aligned}
& \tilde{\Pi}_{I}\left(q^{2}\right)=-\frac{3 i}{2^{11} \pi^{12} q^{2}} \int \frac{d^{4} x d^{4} p_{1} d^{4} p_{2}}{x^{8}\left(p_{1}^{2}-m_{c}^{2}\right)\left(p_{2}^{2}-m_{c}^{2}\right)} e^{i q \cdot x} e^{-i p_{1} \cdot x} e^{-i p_{2} \cdot x}
\end{aligned}
$$

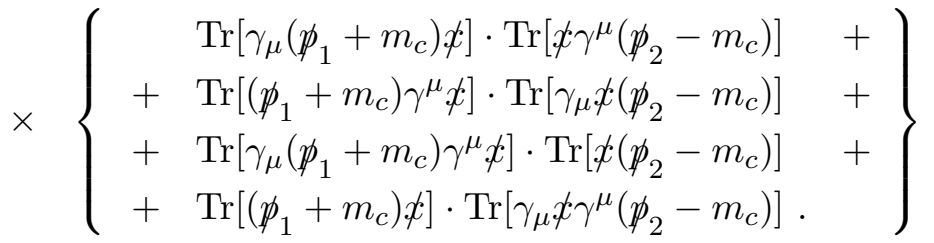

Simplificando e calculando os traços:

$$
\tilde{\Pi}_{I}\left(q^{2}\right)=\frac{3 i}{2^{6} \pi^{12} q^{2}} \int \frac{d^{4} x d^{4} p_{1} d^{4} p_{2}}{x^{8}\left(p_{1}^{2}-m_{c}^{2}\right)\left(p_{2}^{2}-m_{c}^{2}\right)} e^{i q \cdot x} e^{-i p_{1} \cdot x} e^{-i p_{2} \cdot x}\left[2\left(x \cdot p_{1}\right)\left(x \cdot p_{2}\right)+m_{c}^{2} x^{2}\right] .
$$

De forma análoga, para os demais diagramas basta inserirmos o propagador (A.4):

$$
S_{a b}^{q}(x)=-\frac{m_{q} \delta_{a b}}{4 \pi^{2} x^{2}}
$$

para obtermos a correção, em primeira ordem, na massa do quark leve q. As expressões para $\Pi_{I I}\left(q^{2}\right)$ e $\Pi_{I I I}\left(q^{2}\right)$ são dadas respectivamente por:

$$
\begin{aligned}
\Pi_{I I}\left(q^{2}\right) & =-\frac{3 m_{q} m_{c}}{2^{7} \pi^{12} q^{2}} \int \frac{d^{4} x d^{4} p_{1} d^{4} p_{2}}{x^{6}\left(p_{1}^{2}-m_{c}^{2}\right)\left(p_{2}^{2}-m_{c}^{2}\right)} e^{i q \cdot x} e^{-i p_{1} \cdot x} e^{-i p_{2} \cdot x}\left[\left(x \cdot p_{1}\right)-\left(x \cdot p_{2}\right)\right] \\
\Pi_{I I I}\left(q^{2}\right) & =\frac{3 m_{q} m_{c}}{2^{7} \pi^{12} q^{2}} \int \frac{d^{4} x d^{4} p_{1} d^{4} p_{2}}{x^{6}\left(p_{1}^{2}-m_{c}^{2}\right)\left(p_{2}^{2}-m_{c}^{2}\right)} e^{i q \cdot x} e^{-i p_{1} \cdot x} e^{-i p_{2} \cdot x}\left[\left(x \cdot p_{1}\right)-\left(x \cdot p_{2}\right)\right] .
\end{aligned}
$$

Como essas integrais são simétricas pela troca $p_{1} \leftrightarrow p_{2}$, então

$$
\Pi_{I I}\left(q^{2}\right)=\Pi_{I I I}\left(q^{2}\right)=0
$$

Logo, os termos de correção na massa não contribuem para a função de correlação perturbativa das moléculas, assim como não contribuem no caso de tetraquarks. Então:

$$
\tilde{\Pi}^{p e r t}\left(q^{2}\right)=\frac{3 i}{2^{6} \pi^{12} q^{2}} \int \frac{d^{4} x d^{4} p_{1} d^{4} p_{2}}{x^{8}\left(p_{1}^{2}-m_{c}^{2}\right)\left(p_{2}^{2}-m_{c}^{2}\right)} e^{i q \cdot x} e^{-i p_{1} \cdot x} e^{-i p_{2} \cdot x}\left[2\left(x \cdot p_{1}\right)\left(x \cdot p_{2}\right)+m_{c}^{2} x^{2}\right] .
$$


Note que as integrais acima são as mesmas presentes em (C.3). A única diferença entre a função de correlação perturbativa das moléculas com a dos tetraquarks é um fator multiplicativo.

$$
\tilde{\Pi}^{\text {pert }}\left(q^{2}\right)=\frac{3}{4} \Pi^{\text {pert }}\left(q^{2}\right)
$$

Consequentemente, para a densidade espectral também teremos:

$$
\tilde{\rho}^{\text {pert }}\left(q^{2}\right)=\frac{3}{4} \rho^{\text {pert }}\left(q^{2}\right)
$$

Portanto, através destas relações obtemos diretamente a função de correlação e a densidade espectral perturbativa para as moléculas:

$$
\begin{gathered}
\tilde{\Pi}^{p e r t}\left(q^{2}\right)=\frac{1}{2^{10} \pi^{6} q^{2}} \int_{0}^{1} \frac{d \alpha d \beta}{\alpha^{3} \beta^{3}}(1-\alpha-\beta)\left[m_{c}^{2}(\alpha+\beta)-\alpha \beta q^{2}\right]^{3}\left[m_{c}^{2}(1-2 \alpha-2 \beta)+\alpha \beta q^{2}\right] \ln \left[m_{c}^{2}(\alpha+\beta)-\alpha \beta q^{2}\right] \\
\tilde{\rho}^{\text {pert }}(s)=-\frac{1}{2^{10} \pi^{6} s} \int_{\alpha_{\text {min }}}^{\alpha_{\text {max }}} \frac{d \alpha}{\alpha^{3}} \int_{\beta_{\text {min }}}^{1-\alpha} \frac{d \beta}{\beta^{3}}(1-\alpha-\beta)\left[m_{c}^{2}(\alpha+\beta)-\alpha \beta s\right]^{3}\left[m_{c}^{2}(1-2 \alpha-2 \beta)+\alpha \beta s\right]
\end{gathered}
$$

onde $\alpha_{\min }, \alpha_{\max }$ e $\beta_{\min }$ são os mesmos limites mostrados em (C.8) e (C.9).

\section{D.2 Contribuição não-Perturbativa}

Para as contribuições não-perturbativas, tomemos como exemplo o cálculo da função de correlação (3.45) dos condensados de glúons. Para esta contribuição admitimos os seguintes diagramas:

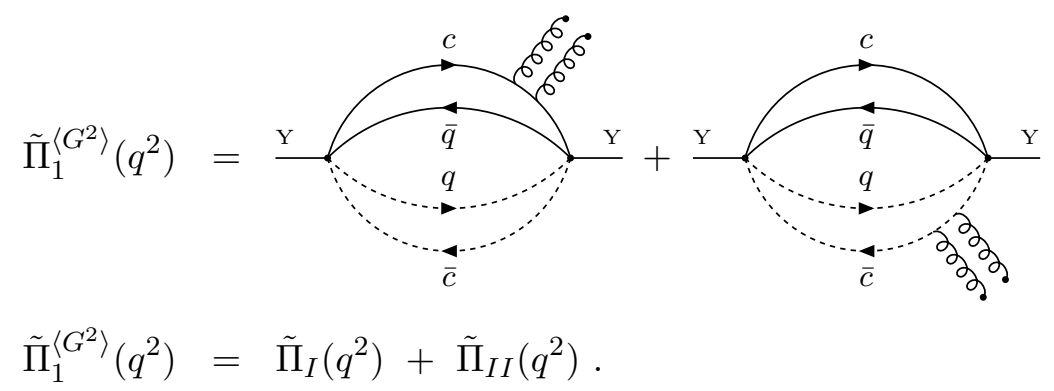

Para o primeiro diagrama, temos que utilizar os propagadores (A.3), (A.12) e (A.14) na linha dos quarks e antiquarks da seguinte forma:

$$
\begin{array}{llrl}
\text { Méson } D_{0}: & S_{a i}^{c}\left(p_{1}\right)=\frac{i \delta_{a i}}{12}\left[\frac{p_{1}^{2}+m_{c} \not p_{1}}{\left(p_{1}^{2}-m_{c}^{2}\right)^{4}}\right] & S_{i a}^{q}(-x) & =-\frac{i \not \not \delta_{i a}}{2 \pi^{2} x^{4}} . \\
\text { Méson } \bar{D}^{*}: & S_{b j}^{q}(x)=\frac{i \not \not \delta_{b j}}{2 \pi^{2} x^{4}} & S_{j b}^{c}\left(-p_{2}\right) & =-\frac{i\left(\not p_{2}-m_{c}\right) \delta_{j b}}{p_{2}^{2}-m_{c}^{2}} .
\end{array}
$$

Inserindo-os na função de correlação (D.1), obtemos: 


$$
\begin{aligned}
& \tilde{\Pi}_{I}\left(q^{2}\right)=-\frac{i m_{c}\left\langle g_{s}^{2} G^{2}\right\rangle}{2^{13} \pi^{12} q^{2}} \int \frac{d^{4} x d^{4} p_{1} d^{4} p_{2}}{x^{8}\left(p_{1}^{2}-m_{c}^{2}\right)^{4}\left(p_{2}^{2}-m_{c}^{2}\right)} e^{i q \cdot x} e^{-i p_{1} \cdot x} e^{-i p_{2} \cdot x} \\
& \times\left\{\begin{array}{rrr}
\operatorname{Tr}\left[\gamma_{\mu}\left(p_{1}^{2}+m_{c} \not p_{1}\right) \not x\right] \cdot \operatorname{Tr}\left[\not \gamma^{\mu}\left(\not p_{2}-m_{c}\right)\right] & + \\
+\operatorname{Tr}\left[\left(p_{1}^{2}+m_{c} \not p_{1}\right) \gamma^{\mu} \not x\right] \cdot \operatorname{Tr}\left[\gamma_{\mu} \not \not\left(\not p_{2}-m_{c}\right)\right] & + \\
+\operatorname{Tr}\left[\gamma_{\mu}\left(p_{1}^{2}+m_{c} \not p_{1}\right) \gamma^{\mu} \not x\right] \cdot \operatorname{Tr}\left[\not \not\left(\not p_{2}-m_{c}\right)\right] & + \\
+\operatorname{Tr}\left[\left(p_{1}^{2}+m_{c} \not p_{1}\right) \not x\right] \cdot \operatorname{Tr}\left[\gamma_{\mu} \not \gamma^{\mu}\left(\not p_{2}-m_{c}\right)\right] . &
\end{array}\right\}
\end{aligned}
$$

Simplificando e calculando os traços:

$$
\tilde{\Pi}_{I}\left(q^{2}\right)=\frac{i m_{c}^{2}\left\langle g_{s}^{2} G^{2}\right\rangle}{2^{8} \pi^{12} q^{2}} \int \frac{d^{4} x d^{4} p_{1} d^{4} p_{2}}{x^{8}\left(p_{1}^{2}-m_{c}^{2}\right)^{4}\left(p_{2}^{2}-m_{c}^{2}\right)} e^{i q \cdot x} e^{-i p_{1} \cdot x} e^{-i p_{2} \cdot x}\left[2\left(x \cdot p_{1}\right)\left(x \cdot p_{2}\right)+x^{2} p_{1}^{2}\right] .
$$

De forma análoga, para o segundo diagrama basta inserirmos em (D.1), o propagador (A.14) na linha do antiquark pesado $c$. A expressão obtida para $\Pi_{I I}\left(q^{2}\right)$ é dada por:

$\Pi_{I I}\left(q^{2}\right)=\frac{i m_{c}^{2}\left\langle g_{s}^{2} G^{2}\right\rangle}{2^{8} \pi^{12} q^{2}} \int \frac{d^{4} x d^{4} p_{1} d^{4} p_{2}}{x^{8}\left(p_{1}^{2}-m_{c}^{2}\right)^{4}\left(p_{2}^{2}-m_{c}^{2}\right)} e^{i q \cdot x} e^{-i p_{1} \cdot x} e^{-i p_{2} \cdot x}\left[2\left(x \cdot p_{1}\right)\left(x \cdot p_{2}\right)+x^{2} p_{1}^{2}\right]$ donde vemos que a contribuição dos dois diagramas são iguais, exatamente como no caso dos tetraquarks. Então:

$$
\Pi_{I}\left(q^{2}\right)=\Pi_{I I}\left(q^{2}\right)
$$

Logo, a função de correlação dos condensados de glúons para as moléculas é dada por:

$$
\tilde{\Pi}_{1}^{\left\langle G^{2}\right\rangle}\left(q^{2}\right)=\frac{i m_{c}^{2}\left\langle g_{s}^{2} G^{2}\right\rangle}{2^{7} \pi^{12} q^{2}} \int \frac{d^{4} x d^{4} p_{1} d^{4} p_{2}}{x^{8}\left(p_{1}^{2}-m_{c}^{2}\right)^{4}\left(p_{2}^{2}-m_{c}^{2}\right)} e^{i q \cdot x} e^{-i p_{1} \cdot x} e^{-i p_{2} \cdot x}\left[2\left(x \cdot p_{1}\right)\left(x \cdot p_{2}\right)+x^{2} p_{1}^{2}\right]
$$

convenientemente, podemos reescrêve-la como:

$$
\tilde{\Pi}_{1}^{\left\langle G^{2}\right\rangle}\left(q^{2}\right)=\frac{i m_{c}^{2}\left\langle g_{s}^{2} G^{2}\right\rangle}{2^{7} \pi^{12} q^{2}} \int \frac{d^{4} x d^{4} p_{1} d^{4} p_{2}}{x^{8}\left(p_{1}^{2}-m_{c}^{2}\right)^{3}\left(p_{2}^{2}-m_{c}^{2}\right)} e^{i q \cdot x} e^{-i p_{1} \cdot x} e^{-i p_{2} \cdot x}\left[\frac{2\left(x \cdot p_{1}\right)\left(x \cdot p_{2}\right)}{p_{1}^{2}-m_{c}^{2}}+x^{2}\left(1+\frac{m_{c}^{2}}{p_{1}^{2}-m_{c}^{2}}\right)\right]
$$

que também pode ser relacionada com (C.13). Como no caso anterior, a única diferença entre a função de correlação dos condensados de glúons das moléculas com a dos tetraquarks é um fator multiplicativo.

$$
\tilde{\Pi}_{1}^{\left\langle G^{2}\right\rangle}\left(q^{2}\right)=\frac{3}{4} \Pi_{1}^{\left\langle G^{2}\right\rangle}\left(q^{2}\right) .
$$

Portanto, através desta relação obtemos diretamente a função de correlação e a densidade espectral dos condensados de glúons para as moléculas:

$$
\begin{gathered}
\tilde{\Pi}_{1}^{\left\langle G^{2}\right\rangle}\left(q^{2}\right)=-\frac{m_{c}^{2}\left\langle g_{s}^{2} G^{2}\right\rangle}{3 \cdot 2^{11} \pi^{6} q^{2}} \int_{0}^{1} \frac{d \alpha d \beta}{\alpha \beta^{3}}(1-\alpha-\beta)\left\{(7 \alpha+3 \beta-3)\left[m_{c}^{2}(\alpha+\beta)-\alpha \beta q^{2}\right]-m_{c}^{2} \alpha(1-\alpha-\beta)\right\} \ln \left[m_{c}^{2}(\alpha+\beta)-\alpha \beta q^{2}\right] \\
\tilde{\rho}_{1}^{\left\langle G^{2}\right\rangle}(s)=\frac{m_{c}^{2}\left\langle g_{s}^{2} G^{2}\right\rangle}{3 \cdot 2^{11} \pi^{6} s} \int_{\alpha_{\text {min }}}^{\alpha_{\max }} \frac{d \alpha}{\alpha} \int_{\beta_{\min }}^{1-\alpha} \frac{d \beta}{\beta^{3}}(1-\alpha-\beta)\left\{(7 \alpha+3 \beta-3)\left[m_{c}^{2}(\alpha+\beta)-\alpha \beta s\right]-m_{c}^{2} \alpha(1-\alpha-\beta)\right\}
\end{gathered}
$$

onde $\alpha_{\min }, \alpha_{\max }$ e $\beta_{\min }$ são os mesmos limites mostrados em (C.8) e (C.9). 


\section{D.3 Contribuição não-Fatorizável}

Para as moléculas, há também a contribuição de termos não-fatorizáveis para os condensados de glúons. Para determiná-los, admitimos os seguintes diagramas:

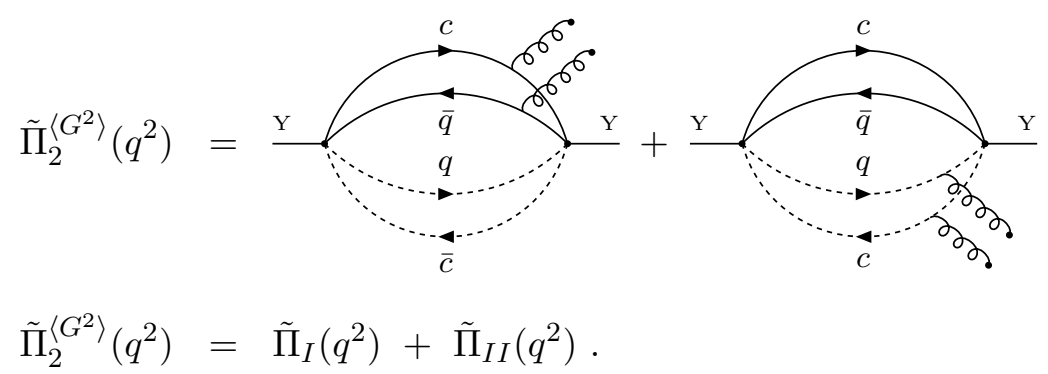

Para o primeiro diagrama, temos que utilizar os propagadores (A.3), (A.9), (A.12) e (A.13) na linha dos quarks e antiquarks da seguinte forma:

$$
\begin{array}{llrl}
\text { Méson } D_{0}: & S_{a i}^{c}\left(p_{1}\right)=-\frac{i t_{a i}^{n}}{4}\left[\frac{\not p_{1} \sigma^{\mu \nu}+\sigma^{\mu \nu} \not p_{1}+2 m_{c} \sigma^{\mu \nu}}{\left(p_{1}^{2}-m_{c}^{2}\right)^{2}}\right] & S_{i a}^{q}(-x) & =\frac{i\left(\not \sigma_{\mu \nu}+\sigma_{\mu \nu} \not\right) t_{i a}^{n}}{3 \cdot 2^{9} \pi^{2} x^{2}}\left\langle g_{s}^{2} G^{2}\right\rangle . \\
\text { Méson } \bar{D}^{*}: & S_{b j}^{q}(x)=\frac{i \not \delta_{b j}}{2 \pi^{2} x^{4}} & S_{j b}^{c}\left(-p_{2}\right) & =-\frac{i\left(\not p_{2}-m_{c}\right) \delta_{j b}}{p_{2}^{2}-m_{c}^{2}} .
\end{array}
$$

Inserindo-os em (D.1), obtemos:

$$
\begin{aligned}
& \tilde{\Pi}_{I}\left(q^{2}\right)=\frac{i\left\langle g_{s}^{2} G^{2}\right\rangle}{3 \cdot 2^{18} \pi^{12} q^{2}} \int \frac{d^{4} x d^{4} p_{1} d^{4} p_{2}}{x^{6}\left(p_{1}^{2}-m_{c}^{2}\right)^{2}\left(p_{2}^{2}-m_{c}^{2}\right)} e^{i q \cdot x} e^{-i p_{1} \cdot x} e^{-i p_{2} \cdot x}
\end{aligned}
$$

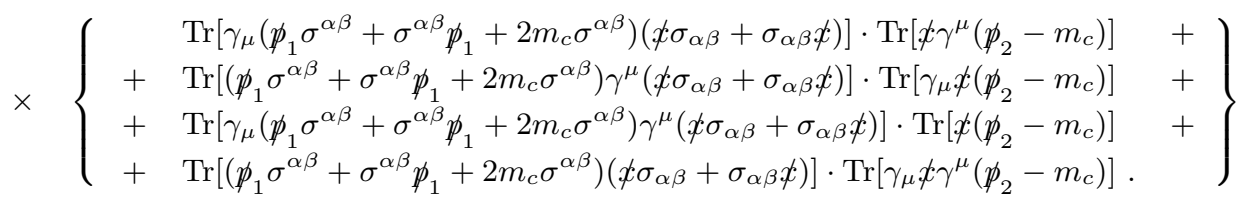

Simplificando e calculando os traços:

$$
\tilde{\Pi}_{I}\left(q^{2}\right)=\frac{i m_{c}^{2}\left\langle g_{s}^{2} G^{2}\right\rangle}{2^{11} \pi^{12} q^{2}} \int \frac{d^{4} x d^{4} p_{1} d^{4} p_{2}}{x^{4}\left(p_{1}^{2}-m_{c}^{2}\right)\left(p_{2}^{2}-m_{c}^{2}\right)} e^{i q \cdot x} e^{-i p_{1} \cdot x} e^{-i p_{2} \cdot x}
$$

De forma análoga, para o segundo diagrama a expressão para $\Pi_{I I}\left(q^{2}\right)$ é dada por:

$$
\Pi_{I I}\left(q^{2}\right)=\frac{i m_{c}^{2}\left\langle g_{s}^{2} G^{2}\right\rangle}{2^{11} \pi^{12} q^{2}} \int \frac{d^{4} x d^{4} p_{1} d^{4} p_{2}}{x^{4}\left(p_{1}^{2}-m_{c}^{2}\right)\left(p_{2}^{2}-m_{c}^{2}\right)} e^{i q \cdot x} e^{-i p_{1} \cdot x} e^{-i p_{2} \cdot x}
$$

donde vemos que a contribuição dos dois diagramas são iguais:

$$
\Pi_{I}\left(q^{2}\right)=\Pi_{I I}\left(q^{2}\right)
$$

Logo, para as moléculas o termo não-fatorizável da função de correlação dos condensados de glúons é dado por:

$$
\Pi_{2}^{\left\langle G^{2}\right\rangle}\left(q^{2}\right)=\frac{i m_{c}^{2}\left\langle g_{s}^{2} G^{2}\right\rangle}{2^{10} \pi^{12} q^{2}} \int \frac{d^{4} x d^{4} p_{1} d^{4} p_{2}}{x^{4}\left(p_{1}^{2}-m_{c}^{2}\right)\left(p_{2}^{2}-m_{c}^{2}\right)} e^{i q \cdot x} e^{-i p_{1} \cdot x} e^{-i p_{2} \cdot x}
$$


Quando comparado com o valor obtido para os tetraquarks (C.19), verificamos que a diferença entre eles é dada por:

$$
\tilde{\Pi}_{2}^{\left\langle G^{2}\right\rangle}\left(q^{2}\right)=\frac{3}{2} \Pi_{2}^{\left\langle G^{2}\right\rangle}\left(q^{2}\right) .
$$

Portanto, através desta relação obtemos diretamente a função de correlação e a densidade espectral não-fatorizáveis dos condensados de glúons para as moléculas:

$$
\begin{gathered}
\tilde{\Pi}_{2}^{\left\langle G^{2}\right\rangle}\left(q^{2}\right)=\frac{m_{c}^{2}\left\langle g_{s}^{2} G^{2}\right\rangle}{2^{10} \pi^{6} q^{2}} \int_{0}^{1} \frac{d \alpha d \beta}{\alpha \beta^{2}} \theta(1-\alpha-\beta)(1-\alpha-\beta)\left[m_{c}^{2}(\alpha+\beta)-\alpha \beta q^{2}\right] \ln \left[m_{c}^{2}(\alpha+\beta)-\alpha \beta q^{2}\right] \\
\tilde{\rho}_{2}^{\left\langle G^{2}\right\rangle}(s)=-\frac{m_{c}^{2}\left\langle g_{s}^{2} G^{2}\right\rangle}{2^{10} \pi^{6} s} \int_{\alpha_{\min }}^{\alpha_{\max }} \frac{d \alpha}{\alpha} \int_{\beta_{\min }}^{1-\alpha} \frac{d \beta}{\beta^{2}}(1-\alpha-\beta)\left[m_{c}^{2}(\alpha+\beta)-\alpha \beta s\right]
\end{gathered}
$$

Por fim, somando as contribuições (D.11) e (D.15), obtemos a contribuição total dos condensados de glúons para a densidade espectral da OPE do estado molecular $D_{0} \bar{D}^{*}$ :

$$
\begin{aligned}
& \tilde{\rho}^{\left\langle G^{2}\right\rangle}(s)=\tilde{\rho}_{1}^{\left\langle G^{2}\right\rangle}(s)+\tilde{\rho}_{2}^{\left\langle G^{2}\right\rangle}(s) \\
& \tilde{\rho}^{\left\langle G^{2}\right\rangle}(s)=\frac{m_{c}^{2}\left\langle g_{s}^{2} G^{2}\right\rangle}{3 \cdot 2^{11} \pi^{6} s} \int_{\alpha_{\min }}^{\alpha_{\max }} \frac{d \alpha}{\alpha} \int_{\beta_{\min }}^{1-\alpha} \frac{d \beta}{\beta^{3}}(1-\alpha-\beta)\left\{(7 \alpha-3 \beta-3)\left[m_{c}^{2}(\alpha+\beta)-\alpha \beta s\right]-m_{c}^{2} \alpha(1-\alpha-\beta)\right\}
\end{aligned}
$$

que é a densidade espectral (3.51) mostrada no Capítulo 3. 


\section{Referências Bibliográficas}

[1] M.E. Peskin e D.V. Schroeder, An Introduction to Quantum Field Theory, Addison-Wesley (1995).

[2] A. Ukawa, Int. J. Mod. Phys. A21, 726-732 (2006); S.J. Dong et al, Phys. Rev. Lett. B605, 137-143 (2005).

[3] M. Gell-Mann, Phys. Rev. Lett. 8:214-2 (1964).

[4] G. Zweig, CERN Preprint 8182/TH401: 24 (1964).

[5] S. Godfrey e N. Isgur, Phys. Rev. D 32, 189 (1985); S. Capstisk e N. Isgur, Phys. Rev. D34, 2809 (1986).

[6] V. Crede e C.A. Meyer, arXiv:0812.0600 [hep-ph] (2008).

[7] T. Barnes, F.E. Close e E.S. Swanson, Phys. Rev. D 52, 5242 (1995).

[8] F.E. Close, arXiv:0706.2709 [hep-ph] (2007).

[9] L. Maiani et al., Phys. Rev. D 71, 014028 (2005).

[10] BaBar Coll., B. Aubert et al., Phys. Rev. D 71, 071103 (2005).

[11] BELLE Coll., X.L. Wang et al., Phys. Rev. Lett. 99, 142002 (2007).

[12] BaBar Coll., B. Aubert et al., Phys. Rev. Lett. 90, 242001 (2003).

[13] CLEO Coll., D. Besson et al., Phys. Rev. D 68, 032002 (2003).

[14] CDF II Coll., D. Acosta et al., Phys. Rev. Lett. 93, 072001 (2004).

[15] D0 Coll., V.M. Abazov et al., Phys. Rev. Lett. 93, 162002 (2004).

[16] W-M Yao et al., J. Phys. G 33, 1 (2006).

[17] BaBar Coll., B. Aubert et al., Phys. Rev. D 74, 071101 (2006).

[18] Belle Coll., K. Abe et al., hep-ex/0505037.

[19] CDF II Coll., D. Abulencia et al., Phys. Rev. Lett. 98, 132002 (2007).

[20] T. Barnes, S. Godfrey e E.S. Swanson, Phys. Rev. D 72, 054026 (2005). 
[21] H. Hogaasen, J.-M. Richard e P. Sorba, hep-ph/0511039.

[22] R.D. Matheus, S. Narison, M. Nielsen e J.-M. Richard, hep-ph/0608297.

[23] D. Bugg, Phys. Lett. B 598, 8 (2004).

[24] B.-A. Li, Phys. Lett. B 605, 306 (2005).

[25] K.K. Seth, Phys. Lett. B 612, 1 (2005).

[26] N. Tornqvist, hep-ph/0308277; F.E. Close e P. Page, Phys. Lett. B 578, 119 (2004).

[27] E.S. Swanson, Phys. Lett. B 588, 189 (2004); Phys. Lett. B 598, 197 (2004).

[28] BaBar Coll., B. Aubert et al., Phys. Rev. Lett. 95, 142001 (2005).

[29] CLEO Coll., Q. He et al., Phys. Rev. D 74, 091104(R) (2006).

[30] L. Maiani et al., Phys. Rev. D 72, 031502 (2005).

[31] BELlE Coll., C.Z. Yuan et al., Phys. Rev. Lett. 99, 182004 (2007).

[32] R. Faccini, arXiv:0801.2679 [hep-ex] (2008).

[33] F.-K. Guo, C. Hanhart e U.-G. Meissner, arXiv:0803.1392 [hep-ph] (2008).

[34] C.F. Qiao, arXiv:0709.4066 [hep-ph] (2007).

[35] G.-J. Ding, J.-J. Zhu e M.-L. Yan, arXiv:0708.3712 [hep-ph] (2007).

[36] E.M. Aitala et al, Phys. Rev. Lett. 86, 770 (2001).

[37] M.A. Shifman, A.I. Vainshtein e V.I. Zakharov, Nucl. Phys. B147, 385 (1979).

[38] R.D. Matheus, S. Narison, M. Nielsen e J.-M. Richard, Phys. Rev. D75, 014005 (2007).

[39] S.H. Lee, A. Mihara, F.S. Navarra e M. Nielsen, Phys. Lett. B661, 28 (2008).

[40] L.J. Reinders, H. Rubinstein e S. Yazaki, Phys. Rept. 127, 1 (1985).

[41] S. Narison, QCD as a theory of hadrons, Cambridge Monogr. Part. Phys. Nucl. Phys. Cosmol. 17, 1 (2002) [hep-h/0205006]; QCD spectral sum rules, World Sci. Lect. Notes Phys. 26, 1 (1989); Acta Phys. Pol. B26, 687 (1995); Riv. Nuov. Cim. 10N2, 1 (1987); Phys. Rept. 84, 263 (1982).

[42] P. Colangelo e A. Khodjamirian, arXiv:hep-ph/0010175 (2000).

[43] V.M. Belyaev, V.M. Braun, A. Khodjamirian e R. Ruckl, Phys. Rev. D51, 6177 (1995). 
[44] R.D. Matheus, Partículas Exóticas em Regras de Soma da QCD, Tese de Doutorado, Instituto de Física - Universidade de São Paulo (2006).

[45] B. L. Ioffe, Nucl. Phys. B188, 317 (1981); B191, 591(E) (1981).

[46] Bagan et al., Nucl. Phys. B254 (1985) 55; D.J. Broadhurst e S. Generalis, Phys. lett. B139 (1984) 85.

[47] S. Narison, Phys. Lett. B466, 345 (1999); S. Narison, Phys. Lett. B361, 121 (1995); S. Narison, Phys. Lett. B387, 162 (1996). S. Narison, Phys. Lett. B624, $223(2005)$.

[48] G.'t Hooft et al, arXiv:0801.2288 (2008).

[49] F.S. Navarra e M. Nielsen, Phys. Lett. B639, 272 (2006).

[50] S.H. Lee, M. Nielsen, U. Wiedner, arXiv:0803.1168 [hep-ph] (2008).

[51] R.M. Albuquerque e M. Nielsen, Nucl. Phys. A815, 53 (2009). 